\title{
Synthetic Advances in the C-H Activation of Rigid Scaffold Molecules
}

\author{
Nitika Grover ${ }^{a}$ \\ Mathias O. Senge*a \\ a School of Chemistry, Trinity College Dublin, The University of \\ Dublin, Trinity Biomedical Sciences Institute, 152-160 Pearse \\ Street, Dublin 2, Ireland \\ sengem@tcd.ie \\ Dedicated to Prof. Dr. Henning Hopf
}
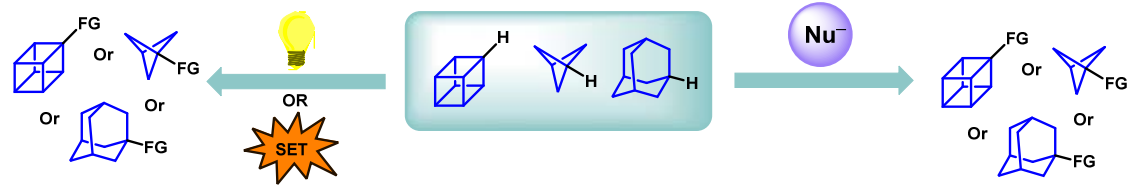

\section{Received: \\ Accepted: \\ Published online: \\ DOI:}

Abstract The remarkable structural and electronic properties of rigid nonconjugated hydrocarbons afford attractive opportunities to design molecular building blocks for both medicinal and material applications. The bridgehead positions provide the possibility to append diverse functional groups at specific angles and in specific orientations. The current review summarizes the synthetic development in $\mathrm{CH}$ functionalization of the three rigid scaffolds namely: (a) cubane, (b) bicyclo[1.1.1]pentane (BCP), (c) adamantane.

1 Introduction

$2 \quad$ Cubane

2.1 Cubane Synthesis

2.2 Cubane Functionalization

$3 \quad$ BCP

3.1 BCP Synthesis

3.2 BCP Functionalization

$4 \quad$ Adamantane

4.1 Adamantane Synthesis

4.2 Adamantane Functionalization

$5 \quad$ Conclusion and Outlook

Key words Cubane, bicyclo[1.1.1]pentane, adamantane, rigid scaffolds, $\mathrm{CH}$ functionalization.

\section{Introduction}

The practice of synthetic organic chemistry of non-natural compounds with rigid organic skeletons provides a deep understanding of bonding and reactivity. In the world of 'impossible' compounds, strained hydrocarbons such as cubane 1, bicyclo[1.1.1]pentane (BCP) 2 and adamantane $\mathbf{3}$ are unique and exhibit interesting but poorly understood chemistry (Figure 1). Initially interest in such rigid moieties arose from the curiosity of organic chemists in exploring new classes of strained molecules. ${ }^{1}$ In part, this was based on their aesthetic beauty and the fundamental challenge; e.g., it is still an open task to synthesize all five hydrocarbon analogues of the platonic bodies (Figure 1). ${ }^{2}$ Even the ones achieved, present a formidable challenge to synthetic chemists. One may only recall the epic endeavors involved in Eaton's cubane synthesis, ${ }^{3}$ Parquette's preparation of dodecahedrane, Prinzbach's pagodane route thereto, or Maier's synthesis of tetra-tert-butyltetrahedrane. ${ }^{4}$

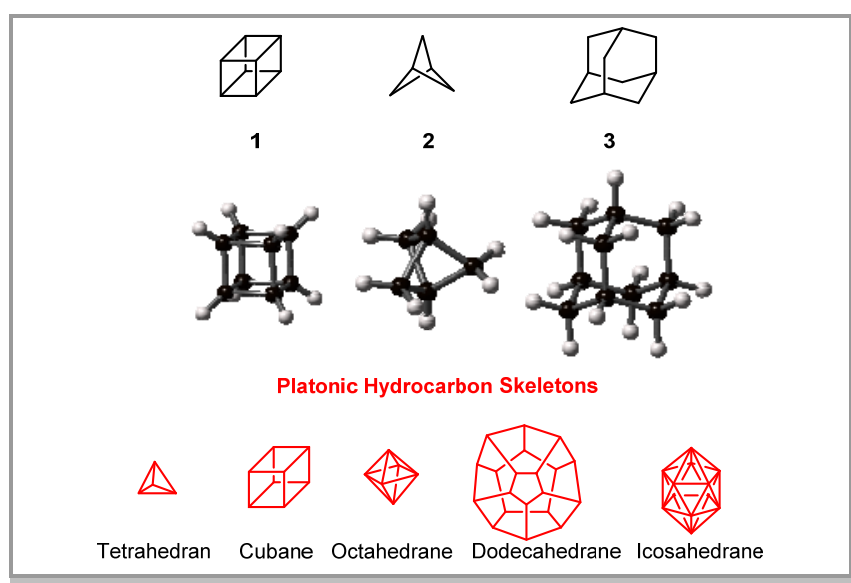

Figure 1 The structures of cubane, BCP and adamantane and the five platonic hydrocarbon systems.

The fascinating architecture of these systems significantly differs from scaffolds realized in natural compounds. Hence, they attracted considerable attention from synthetic and physical organic chemists to investigate their properties and to establish possible uses. ${ }^{3,5}$ Significantly, over the past twenty years, some of these motifs have shown potential in medicinal as well as material chemistry. For example, more than 135 patents for BCPs and cubane have been reported till 2020.6 Moreover, aminoadamantane derivatives such as amantadine, rimantadine, tromantadine and saxagliptin successfully appeared in the pharmaceutical market and most of them are still in use. ${ }^{7}$ In a similar vein, cubane, BCP (bicyclo[1.1.1]pentane), and BCO (bicyclo[2.2.2]octane) units are utilized in the modern context of the "Escape from Flatland". ${ }^{8}$ This bioisostere concept ${ }^{9}$ of replacing, e.g. planar (2D) benzene units with '3D-structured' aliphatic hydrocarbon 
systems of similar 2D dimension has been well documented in the literature. ${ }^{10,11}$

From being strained, and thermally stable, saturated hydrocarbon systems have also attained considerable attention in material science, including the construction of molecular rods and rotors. ${ }^{5 a, 12}$ Giant molecules and supramolecular structures based on small geometrically defined units have long fascinated chemists and might be useful in biochemical and material science applications where small entities cannot be employed. ${ }^{13}$

Furthermore, theoretically and experimentally, the utility of rigid scaffolds in electron transfer system has been shown. They inhibit the undesired overlap of $\pi$-electron clouds while still providing a well-defined spatial arrangement of chromophores, e.g., for through-space electron transfer studies. ${ }^{14}$ Such systems also open the possibility to use rigid hydrocarbon linker units as molecular resistors ${ }^{15 a}$ and building blocks in organic electronics/molecular circuitry. ${ }^{15}$ While the benefits of incorporation of rigid scaffolds are evident, a substantial obstacle to applying this strategy is the lack of synthetic methods to access the desired building blocks.

Indeed, practical and scalable methods must be developed prior to rigid hydrocarbons being able to reach their full potential. This requires chemical transformations at the bridgehead carbons. Despite the extensive success of, for example, transition-metal-catalyzed cross-coupling methodologies, significant limitations still exist for reactions at strained aliphatic carbons. ${ }^{16}$ Most $\mathrm{C}-\mathrm{H}$-functionalization approaches rely on a pre-functionalized organometallic substrate and halogenated coupling partner. ${ }^{17}$ Yet, only the use of non-conventional functional groups (e.g., carboxylic acids

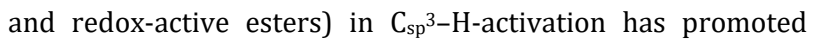
advancements for systems such as cubanes. ${ }^{18}$

The field is rapidly advancing, which makes it possible to finally consider applications for molecules such as those shown in Figure 1 that go beyond the use of simple undergraduate chemistry, e.g., $-\mathrm{C}=\mathrm{O},-\mathrm{COOH},-\mathrm{OH}$ or $-\mathrm{NH}_{2}$ functional group transformations. Hence, this contribution focuses on advances in synthetic chemistry of non-conjugated hydrocarbon linkers with three saturated skeletons; namely: (a) cubane 1, (b) BCP 2, and (c) adamantane 3. Both, the cubane and BCP scaffolds are rigid and strained, ${ }^{19 a}$ the $\mathrm{C}-\mathrm{H}$-activation at the bridgehead positions in these scaffolds is mainly facilitate via single electron transfer (SET) or radical cross-coupling (RCC). In contrast, adamantane is a rigid but strain-free scaffold; ${ }^{19 b}$ thus, the $\mathrm{C}$-H-activation proceeds via standard chemical transformations such as Friedel-Crafts or 'Pd-catalyzed' reactions.

\section{Cubane}

\subsection{Cubane Synthesis}

In 1953, Weltner suggested that the synthesis of cubane may not be possible due to the thermodynamic instability of the cubic moiety compared to its cyclooctatetraene isomers. ${ }^{20}$ Yet, in 1961 Freedman reported the formation of unidentified colorless hydrocarbons during the thermal reaction of the tetraphenylcyclobutadiene nickel bromide complex. ${ }^{21}$ Shortly afterwards this colorless compound was identified as octaphenylcubane. Along the same lines, the formation of octaphenylcubane was observed upon the thermal reaction of diphenylacetylene and phenylmagnesium bromide. ${ }^{22}$

The first practical entry into cubane chemistry was the synthesis of the cubane-1,4-dimethyl ester (10) (Scheme 1) reported by Eaton and Cole in 1964.23 The fundamental features of Eaton's synthetic pathway were (i) endo-selective $[4 \pi+2 \pi]$ dimerization of the 2-bromocyclopentedienone (6A), (ii) photochemical $[2 \pi+2 \pi]$ dimerization of (7), and (iii) double Favorskii ring contraction of the homocubyl moiety (8).

In 1970, Chapman and coworkers introduced several modifications in Eaton's original protocol. ${ }^{24}$ The most recent protocol for the synthesis of dimethylcubane-1,4-dicarboxylate (10) was reported by Tsanaktsidis et al. which incorporates the key features of Eaton's and Chapman's synthetic procedures in eight synthetic steps (Scheme 1b). ${ }^{25}$ This procedure is robust and the easiest way to access dimethylcubane-1,4-dicarboxylate (10) on a multi-gram scale.

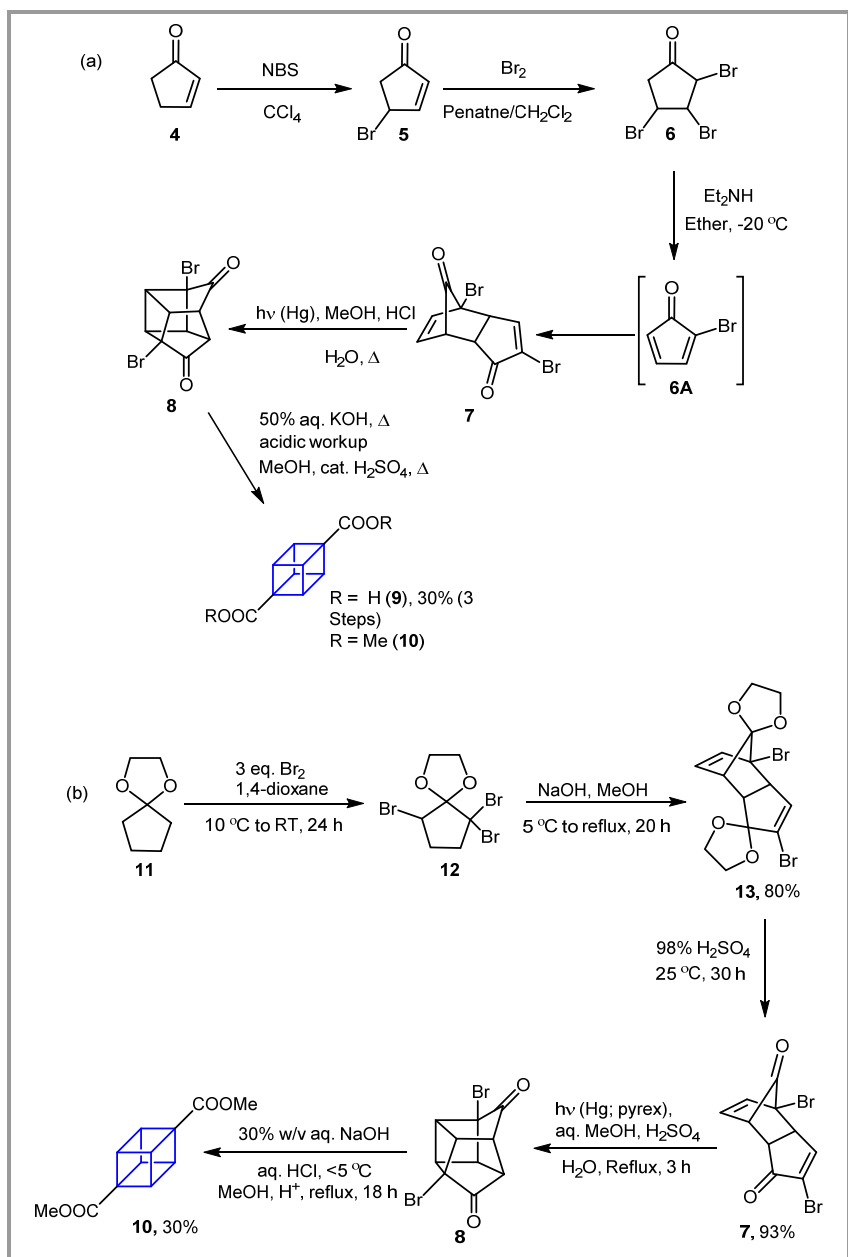

Scheme 1. (a) Eaton's synthesis of dimethylcubane-1,4-dicarboxylate. (b) Contemporary pilot scale synthesis of dimethylcubane-1,4-dicarboxylate. 


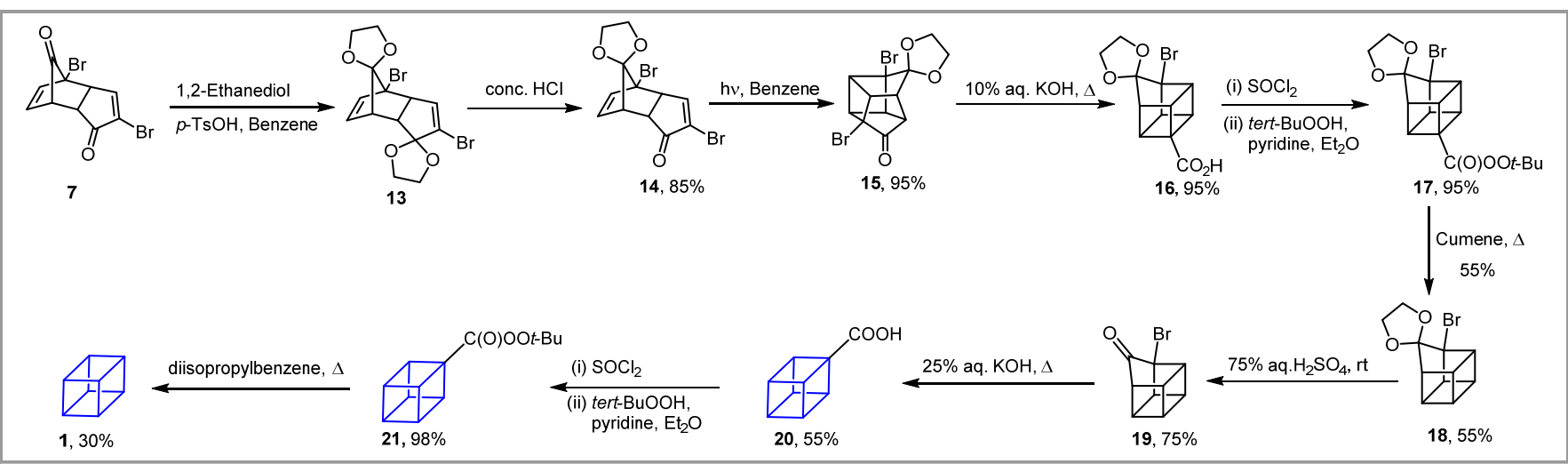

Scheme 2 Eaton and Cole's cubane synthesis.

Shortly after their report on the cubane diester (10) Eaton and Cole completed the first formal synthesis of cubane (1). ${ }^{26}$ As shown in Scheme 2 it involved the stepwise or mono deprotection of the bisketal (13) followed by UV light-mediated $[2 \pi+2 \pi]$ cyclization. Favorskii ring contraction of the cyclized moiety (15) afforded the carboxylic acid derivative of homocubyl skeleton (16), which undergoes reductive decarboxylation to afford the monoketalcubnyl system (18). Similar synthetic steps were repeated to access the first unsubstituted cubane (1) (Scheme 2).

Subsequently, a range of octa- or persubstituted cubanes such as octa(trifluoromethyl)cubane, octacyclopropylcubane and octamethylcubane became accessible although they remained less explored due to limited synthetic strategies. ${ }^{27,28,29}$ To date this still holds true for many aspects of cubane chemistry, notably in terms of $\mathrm{C}-\mathrm{H}$-activation. As will be briefly outlined below, most of the successful cubane functionalization methods rely heavily on the interconversion of dimethylcubane-1,4dicarboxylate (10) or cubane-1,4-dicarboxylic acid (9) due to their comparatively easy availability as starting materials via conventional syntheses. Hence, a variety of synthetic pathways have been established for interconversion of ester and acid groups into other functional groups $\left(\mathrm{NO}_{2}, \mathrm{CHO}, \mathrm{NHCO}\right.$, ethylene, etc.) which allow the use of the cubane system in bioisostere, ${ }^{30}$ medicinal ${ }^{31}$ and supramolecular chemistry. ${ }^{32}$

\subsection{Cubane Functionalization}

Despite its fundamental relevance and the necessity to understand the electronic and steric properties of hydrocarbon skeletons, it was not until Eaton raised the specter of cubanes as potential explosives 33 and formulated his hypothesis of bioisosteres ${ }^{9}$ that cubane chemistry experienced a renaissance. Cubane is a high energy material and its strain energy, thermal, and shock stability strongly depends on the type of functional groups present. For example, cubane-1,4-dicarboxylic acid (9), dimethylcubane-1,4-dicarboxylate (10), and amide derivates are sensitive to shock, whereas halo substituted cubanes detonate upon strong heating. ${ }^{33 c}$ In terms of functionalization, the cubane skeleton (1) has proven resistant to many reactions including nitration, formylation, and $\mathrm{C}-\mathrm{H}$ activation and thus synthetic chemists proceed with caution. On the other hand, dimethylcubane-1,4-dicarboxylate (10), as the prerequisite for classical functional group interconversion chemistry, is highly relevant and has found extensive use in the past five and a half decades. The development of a simple five-step synthetic procedure (Scheme 1b) promoted the industrial-scale production of dimethylcubane-1,4-carboxylate (10). Several companies including Fluorochem in California, EniChem in Milan, and Boron Molecular in Australia conduct the synthesis on a multi-kilogram scale in small pilot plants. In the following we describe the synthesis of substituted cubanes via the interconversion of ester or acid group/s.

\subsubsection{Nitrocubanes}

The high density and strain energy of nitrocubanes make them a potential hallmark in the field of high energy materials and explosives. $^{34}$ In the 1980s, the U.S. Army Armament and Development Command identified nitrocubane derivatives as potential non-nuclear explosives. Furthermore, theoretical calculations by Sandus and Alster pointed out that octanitrocubane (31) might be $20-25 \%$ more effective than HMX/octogen. ${ }^{35}$ The introduction of nitro group/s to the cubane skeleton was a challenging task and, was again achieved by Eaton and coworkers (Scheme 3). ${ }^{33,36,37}$

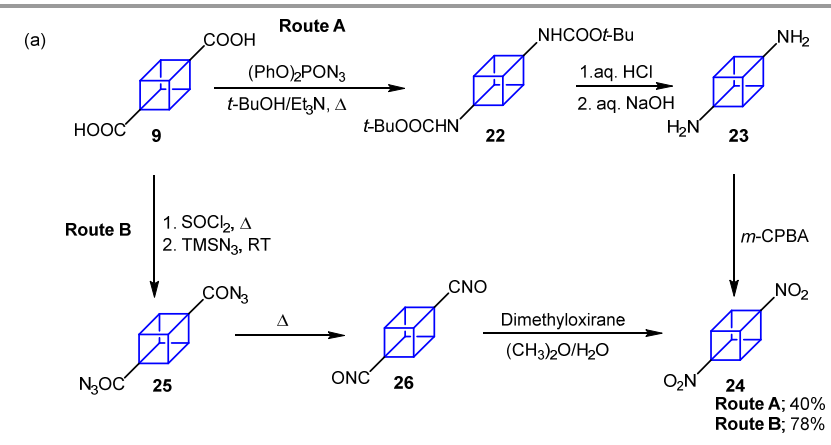

(b)

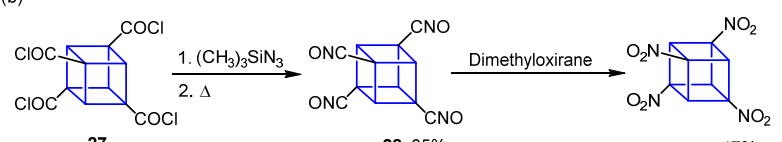
$27 \mathrm{COCl}, 85 \%$ 2. $\left.\mathrm{NaN}_{2} \mathrm{~S}_{4}\right|_{\mathrm{NO}_{2}} 29,47 \%$

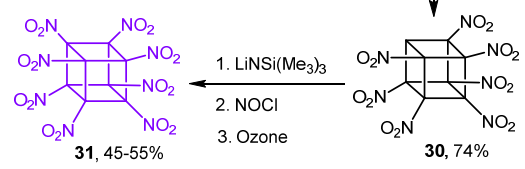

Scheme 3 (a) Synthesis of 1,4-dinitrocubane. (b) Synthesis of tetra-, hepta-, and octanitrocubanes. 
Initial attempts to convert cubane-1,4-carboxylic acid (9) to cubane-1,4-diaminocubane (23) via the Curtius rearrangement of cubane-1,4-dicarbonyl diazide (25) failed due to the explosive nature of the azide intermediate. However, the treatment of cubane-1,4-dicarboxylic acid (9) with phenylphosphoryl azide and triethylamine in refluxing tertbutyl alcohol produced 1,4-bis[(tert-butoxycarbonyl)amino]cubane (22). Further transformation of this key intermediate (22) involved hydrolysis followed by decarboxylation and provided access to 1,4-diaminocubane (23). ${ }^{36}$ The unstable and crude 1,4-diaminocubane (23) was oxidized to 1,4-dinitrocubane (24) using $m$-chloroperbenzoic acid (Scheme 3a; Route A). Unfortunately, this peracid oxidation resulted in low yields and the yields further deteriorated upon scale-up. A few years later, Eaton and Wicks developed a modified protocol, commencing from cubane-1,4-dicarboxylic acid. The addition of thionyl chloride $\left(\mathrm{SOCl}_{2}\right)$ and trimethylsilyl azide $\left(\mathrm{TMSN}_{3}\right)$ to the carboxylic acid gave the isocyanate (26), which was further oxidized using dimethyldioxirane in wet acetone to yield 1,4-dinitrocubane (24) (Scheme 3a; Route B). ${ }^{37}$

A similar strategy was followed for the synthesis of 1,3,5,7tetranitrocubane (29). ${ }^{38}$ Bashir-Hashemi developed photochemical chlorocarbonylation to introduce two more carbonyl moieties at the cubane skeleton. ${ }^{39}$ However, this reaction is quite sensitive to the light source and reaction time. In addition, the products often are a regioisomeric mixture and thus, it cannot be performed with precision. Regardless of the aforementioned shortcomings, this reaction is one of the most convenient approaches to synthesize tetramethylcubane1,3,5,7-tetracarboxylate. ${ }^{39} \quad$ Tetramethylcubane-1,3,5,7tetracarboxylate was subsequently converted into cubane1,3,5,7-tetracarbonyl tetrachloride (27). The reaction of $\mathbf{2 7}$ with trimethylsilyl azide $\left(\mathrm{TMSN}_{3}\right)$ yielded compound $\mathbf{2 8}$; subsequently, in situ oxidation of $\mathbf{2 8}$ afforded 1,3,5,7tetranitrocubane (29) (Scheme 3b).

Tetranitrocubane (29) is highly acidic, which proved to be a key aspect in the preparation of penta-, hexa-, and heptanitrocubane. The reaction of sodium bis(trimethylsilyl)amide $\quad\left(\mathrm{NaN}\left(\mathrm{SiMe}_{3}\right)_{2}\right) \quad$ with $\quad 1,3,5,7-$ tetranitrocubane generates the cubane carbanion, which was further nitrated using $\mathrm{N}_{2} \mathrm{O}_{4}$. The carbanion of heptanitrocubane (30) was too unreactive to nitrate with $\mathrm{N}_{2} \mathrm{O}_{4}$, therefore, a more powerful oxidizing agent nitrosyl chloride ( $\mathrm{NOCl}$ ) was added to lithiated heptanitrocubane. Ozonation of the intermediate resulted in octanitrocubane (31) (Scheme 3b). ${ }^{33}$ Octanitrocubane (31) was theoretically claimed as a powerful non-nuclear explosive; however, the shock insensitivity and thermal stability of this molecule makes it impractical as an explosive.

\subsection{2 lodocubanes and their Transformations}

The introduction of halogen atoms at the cubane skeleton offers an interesting approach for further functionalization. Moriarty and coworkers developed a unique means to access 1iodocubane (32). Cubane-1-carboxylic acid (20) was treated with (diacetoxyiodo)benzene-carbon tetrachloride-iodine $\left(\mathrm{Phl}(\mathrm{OAc})_{2}-\mathrm{CCl}_{4}-\mathrm{I}_{2}\right)$ under irradiation conditions to afford 1iodocubane (32) in 80-90\% yields (Scheme $4 a$ ). ${ }^{40}$ The synthesis of 1,4-diiodocubane was achieved using Barton's decarboxylative iodination strategy ${ }^{41}$ and involved the reaction of cubane-1,4-dicarbonyl dichloride with $N$-hydroxypyridine-2thione and trifluoroiodoethane in the presence of a catalytic amount of $\mathrm{N}, \mathrm{N}$-dimethylaminopyridine (Scheme $4 \mathrm{~b}$ ). ${ }^{42}$ Additionally, Barton decarboxylation of 4-substituted cubane-1carboxylic acids (33-35) in carbon tetrachloride or 1,1,1trifluoro-2-iodoethane gave access to 4-iodo or 4-chlorocubane derivatives.

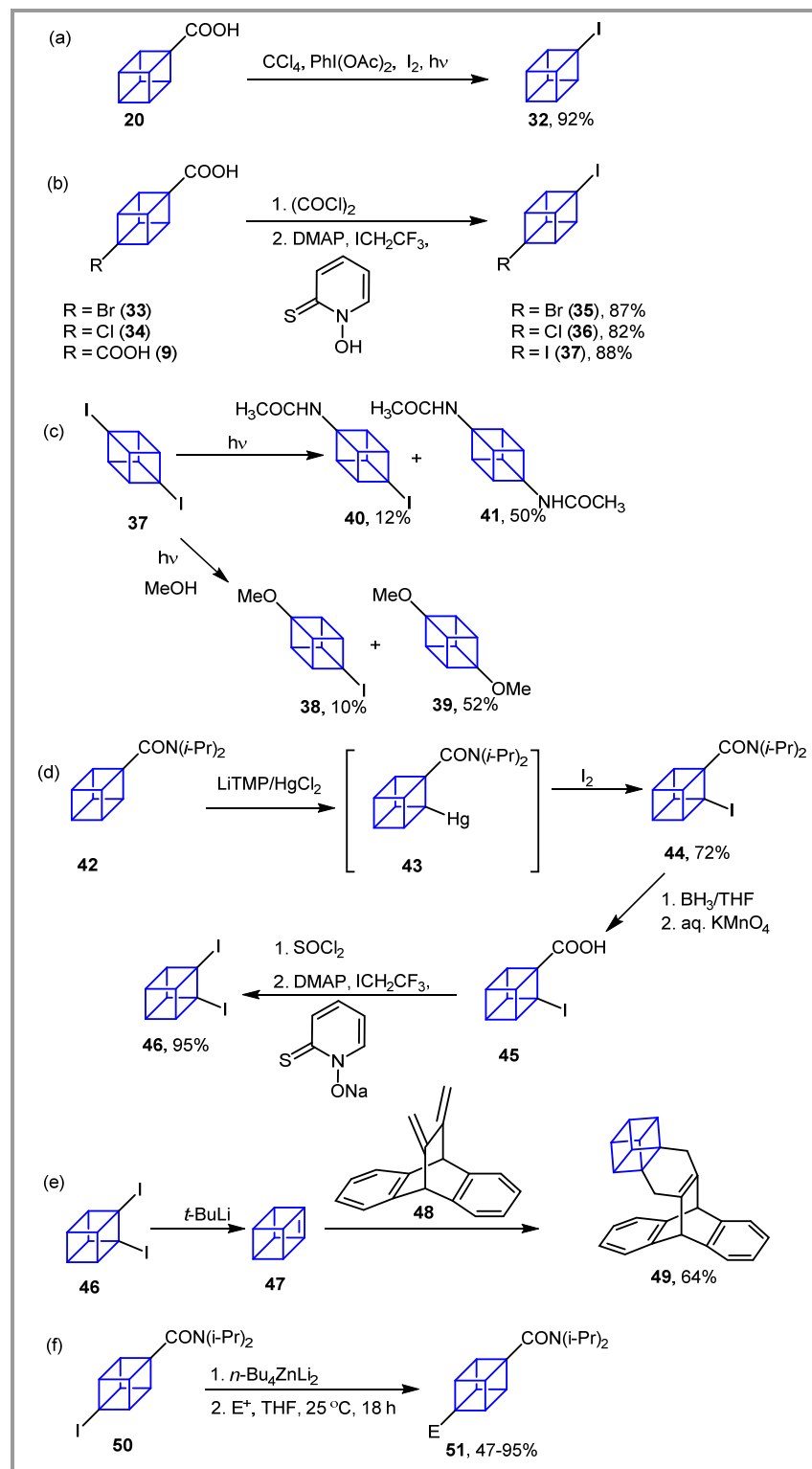

Scheme 4 (a) Synthesis of 1-iodocubane. (b) Synthesis of 4-halo-1-iodo cubane. (c) Photolysis of 1,4-diiodocubane. (d) Synthesis of 1,2-diiodocubane. (e) Synthesis of cubene and its utility in Diels-Alder reaction. (f) functionalization of cubane using lithium organozincate reagent.

The susceptibility of the cubane core towards nucleophilic attack has been reported by various research groups across the globe. 1,4-Diiodocubane (37) was further utilized for the synthesis of anisoles $(\mathbf{3 9}, \mathbf{4 0})$ and acetamide isosteres $(\mathbf{4 1}, \mathbf{4 2})$. Photolysis of a $0.03 \mathrm{M}$ solution of 1,4-diiodocubane in methanol afforded the 1 -iodo-4-methoxycubane $(\mathbf{3 8})$ in a $10 \%$ yield. ${ }^{43 a}$ Subsequently, efforts by Irngartinger et al. improved the photolysis yield and it reached 58\%. Similarly, irradiation of 1,4- 
diiodocubane in wet acetonitrile yielded the acetamide derivative (41) in an overall yield of $12 \%$ (Scheme $4 \mathrm{c}$ ). ${ }_{4}^{43 \mathrm{~b}}$

Iodine-metal exchange reactions on the cubane skeleton were examined using several metal derivatives, including magnesium, tin, zinc, lithium, and mercury. Transmercuration of lithiatedcubane was applied to the synthesis of 1,2-diiodocuabe (46). The treatment of $N, N$-diisopropylcarboxamide cubane (42) with an excess of lithium tetramethylpiperidide/mercury(II) chloride ( $\mathrm{LiTMP} / \mathrm{HgCl}_{2}$ ) generated 2-mercury-1-( $N, N$-diisopropylcarboxamide)cubane (43), which gave 2-iodo-1- $(N, N$ diisopropylcarboxamide)cubane (44), following treatment with molecular iodine. The resultant iodocubanecarboxamide was treated with $\mathrm{BH}_{3} \bullet \mathrm{TMS}$, followed by aq. $\mathrm{KMnO}_{4}$, to generate 2 iodocubane-1-carboxylic acid (45). In the last step, the $\mathrm{COOH}$ group was converted to an iodo group using a modified version of Barton's decarboxylative iodination strategy (Scheme 4d). The reaction of 1,2-diiodocubane (46) with tert-BuLi resulted in the formation of cubene (47); this immensely strained olefin has previously been used as a building block in Diels-Alder reactions (Scheme 4e). To date, cubene is one of the most pyramidalized olefins described. ${ }^{44}$

Organolithoium reagents account for significant progress in synthetic organic chemistry. The combined basicity and nucleophilicity of organolithium reagents make them one of the most important classes of organallometallic compounds. ${ }^{45 a}$ The reactivity of halogen substituted cubanes towards alkyl- and aryl-lithium reagents has been explored. ${ }^{45 b, 46}$ Phenyllithium reacts with 1-iodo-4-halocubanes $(35,36)$ to yield the phenyl cubane. This reaction proceeds via a diradical mechanism. However, the reaction of alkyl lithium with iodocubane did not proceed smoothly and the synthesis of alkyl-substituted cubanes is more difficult than the aryl analogs. For example, the reaction of iodocubane with MeLi produced mostly starting material, i.e. iodocubane. ${ }^{46}$ As discussed above, iodocubanes do not react with strong alkyllithium reagents. Additionally, cubyllithium does not act as a strong nucleophile in the presence of certain electrophiles due to its instability and high basicity. Similarly, the reaction of 1,4-diiodocubane with an alkyl-Grignard reagent resulted in products with various substitution patterns and the formation of cubylcubane derivatives was observed. ${ }^{47}$ Hence, another organometallic reagent was required to functionalize iodocubanes. The most recent report by Kato et al. describes the utilization of a lithium oraganozincate reagent for metal-iodine exchange. 4Iodocubanecarboxamide was treated with lithium organozincates $\left(n-\mathrm{Bu}_{4} \mathrm{ZnLi}_{2}\right)$ in THF at $25{ }^{\circ} \mathrm{C}$ to yield the cubylmetal species. In the next step, this intermediate was treated with a range of electrophiles to give the substituted cubanes (Scheme 4f). 48

Unfortunately, Pd catalyzed coupling reactions using iodo, tin, zinc, and silyl derivatives of cubane were quite ineffective, even after several optimization attempts including varying the substrates, reagents, temperature, and catalysts. ${ }^{49}$

\subsubsection{Alkynylcubanes}

Due to their linear and well-defined dimensionality, alkynylsubstituted saturated cyclic hydrocarbons are important building blocks in the developing field of rigid-rod arrays. ${ }^{50}$ In
1986, Negishi and coworkers developed a mild approach to access terminal alkynes from methyl ketones. ${ }^{51}$ Initially, alkynyl cubanes $(\mathbf{5 6 , 5 7})$ were synthesized from diethyl phosphate enolates of cubyl methyl ketones $(\mathbf{5 4 , 5 5})$ using a modified Negishi approach (Scheme 5a). However, the desired products were obtained in only 5-32\% yields, which limited the use of Negishi's protocol. ${ }^{52}$

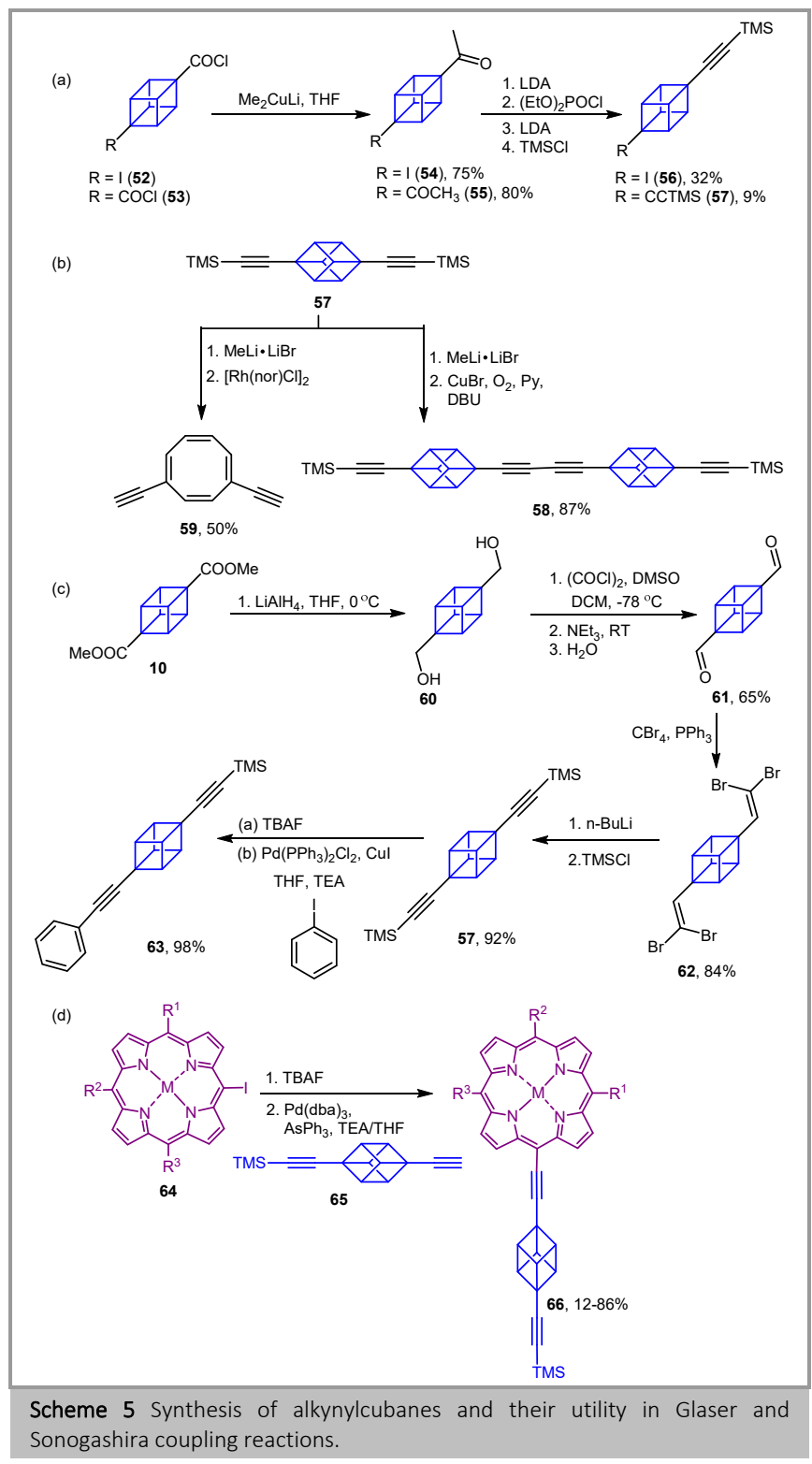

Moreover, the synthesized ethynyl cubanes were successfully subjected to homocoupling or Glaser coupling reactions to yield the dimer 58. $\mathrm{Rh}(\mathrm{I})$ catalyzed coupling reactions with these derivatives opens the cubane skeleton to alkynyl substituted cyclooctatetraene (COT) (59) (Scheme 5b,c). ${ }^{53}$ Later, Eaton et al. applied the Corey-Fuchs procedure to synthesize 1,4dialkynylcubane, which proved to be very successful. Dimethyl cubane-1,4-dicarboxylate (10) was transformed into cubane1,4-diyldimethanol (60) and cubane-1,4-dicarbaldehyde (61) using standard chemical reactions. Cubane-1,4-dicarbaldehyde (61) was employed as a key compound to afford compound 57 in three steps. Furthermore, Sonogashira coupling was attempted using bis(triphenylphosphine)palladium(II) chloride 
$\left(\mathrm{Pd}\left(\mathrm{PPh}_{3}\right)_{2} \mathrm{Cl}_{2}\right)$, copper(I) bromide $\left(\mathrm{Cu}_{2} \mathrm{Br}_{2}\right)$ and triethyl amine (TEA). However, the use of $\mathrm{Cu}_{2} \mathrm{Br}_{2}$ as a $\mathrm{Cu}(\mathrm{I})$ source is not practical and the authors presented a limited substrate scope without a detailed optimization study. ${ }^{53}$ Thus, there was a need for an in-depth Sonogashira coupling reaction study on the cubane skeleton. In 2018, our group showed that 1,4ethynylcubane is surprisingly stable in the presence of $\mathrm{Pd}$ catalysts. The reaction of $\mathbf{5 7}$ with 4-iodobenzene in the presence of $\mathrm{Pd}\left(\mathrm{PPh}_{3}\right)_{4}, \mathrm{CuI}$, and trimethylamine (TEA) yielded 63. Similarly, the reaction of 1-iodo-4-ethynylcubane with iodobenzene in the presence of tetrakis(triphenylphosphine)palladium $\left(\mathrm{Pd}\left(\mathrm{PPh}_{3}\right)_{4}\right)$, copper(I) iodide (CuI), and trimethylamine (TEA) proceeded readily with an isolated yield of $98 \%$ (Scheme $5 \mathrm{c}$ ). Furthermore, delicate but fascinating cubane-porphyrin arrays (66) were synthesized using the $\mathrm{Cu}$-free Sonogashira coupling of mono- (65) or bisethynyl cubane (57) and iodo-porphyrin (64) (Scheme 5d). ${ }^{54}$

\subsubsection{Radical Cross-coupling or SET Reaction on the Cubane Skeleton}

The high thermal stability of cubane contradicts WoodwardHoffmann's orbital symmetry rules. ${ }^{55}$ However, rearrangements of the cubane skeleton occur in the presence of catalytic amounts of transition metals. In the presence of $\mathrm{Rh}(\mathrm{I})$ salts, cubane rearranged to syn-tricyclooctadiene, whereas Pd(II)- or $\mathrm{Ag}(\mathrm{I})$-catalyzed reactions led to the formation of cuneane. ${ }^{56}$ The failure of transition metal-catalyzed coupling reactions on the cubane skeleton has limited the use of halogenated cubane derivatives. Therefore, a better approach is required to append aryl groups directly on to the cubane skeleton. Interestingly, the cubane biradical is highly reactive and exists in a singlet state, which is unreactive toward Diels-Alder dienes. ${ }^{57}$ Hence, the cubane radical is a potential key intermediate for direct functionalization on the skeleton.

The first cubane aryl system was achieved using the light-driven reaction of cubane carboxylic acid with lead acetate and benzene. ${ }^{58}$ The proposed reaction mechanism described the formation of a lead cubane carboxylate complex, the next step involved decarboxylation followed by the generation of the cubane radical. The cubane radical then reacts with benzene to access the aryl cubane. However, the substrate scope for this reaction was limited. In 1991, Okada et al. reported a Michael addition reaction via the photo-decarboxylation of $\mathrm{N}$ acetoxyphthalamides. This method was the first report of the use of redox-active esters in radical coupling reactions. ${ }^{59}$

In further synthetic endeavors, single-electron chemistry or radical cross-coupling (RCC) reactions were proven as promising tools for the functionalization of the cubane framework. The selective monohydrolysis of dimethylcubane1,4-dicarboxylate (10) to yield 4-(methoxycarbonyl) cubane-1carboxylic acid (67) proceed smoothly in a 95\% yield. Readily available 4-(methoxycarbonyl) cubane-1-carboxylic acid (67) was converted into redox-active esters such as $\mathrm{N}$ hydroxyphthalimide (NHPI) (68) or tetrachloro- $N$ hydroxyphthalimide (TCNHPI) (69) using standard amide coupling conditions. ${ }^{54,60}$ These NHPI or TCNHPI compounds were identified as superior surrogates for alkyl halides in various Ni or Fe catalyzed coupling reactions (Scheme 6; Route A and Route B)
These esters $(\mathbf{6 8}, \mathbf{6 9})$ undergo a rapid decarboxylation via oneelectron reduction, which subsequently generates an alkyl radical. In the next step, the alkyl radical forms a C-C bond by either reacting with an electrophilic olefin or undergoing a Suzuki-Miyaura-type coupling reaction, mediated by a transition metal, with a carbon-centered organometallic nucleophile, e.g., a boronic ester. Baran and coworkers documented the synthesis of $\mathbf{7 0}$ using coupling of redox-active esters (68) with diphenylzinc species using a Fe-based catalyst $\left(\mathrm{Fe}(\mathrm{acac})_{3} / \mathrm{dppBz}\right.$ ) system (Scheme 6; Route A). ${ }^{60}$ Following this, our group was able to improve the yields of RCC reactions by reacting cubaneTCNHPI (69) with $\mathrm{Ar}-\mathrm{ZnCl} \bullet \mathrm{LiCl}$ in the presence of a Ni-catalyst $\left(\mathrm{NiCl}_{2} \bullet\right.$ glyme/bipy) (Scheme 6; Route B). ${ }^{54}$ This method allowed the synthesis of variety of aryl substituted cubanes (71). Additionally, the direct attachment of a porphyrin unit to the cubane skeleton was achieved albeit in low yield. Interestingly, the photosensitized decarboxylation of cubane NHPI, and the transformation of the carboxylic ester group into an isoelectronic boronic ester (72), was also achieved by Agarwal's group (Scheme 6; Route C). ${ }^{61}$

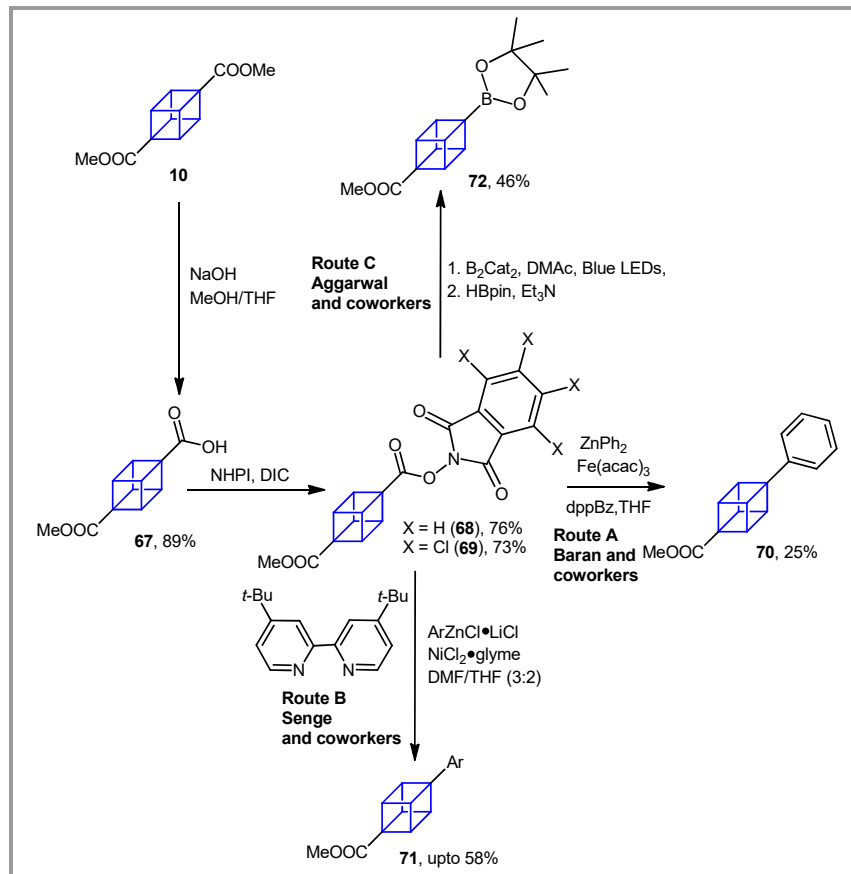

Scheme 6 Synthesis of cubane redox-active esters and subsequent redox coupling reactions.

The viability of cubyl radicals for cross-coupling is supported by the examples described above. Moreover, the solvolysis of iodocubanes is supported by the formation of cationic species. The possibility of cubane cation and/or radical formation promotes electrosynthetic investigations on cubane derivatives. Along the same line, the electrochemical functionalization of 4substituted-cubane-1-carboxylic acids $(\mathbf{3 3 , 3 4 , 6 7 )}$ was reported.62 The electrolysis of 4-substituted-cubane-1carboxylic acids $(33,34,67)$ initially formed the radical, which was oxidized further to yield the carbocation. The resultant carbocation reacted with nucleophiles (alcohols) to afford cubane derivatives. The anodic oxidation of 4(methoxycarbonyl)cubane-1-carboxylic acid (67) using a C/PVDF anode (200 $\mathrm{mA}, 0.5 \mathrm{~mL} / \mathrm{min})$ in the presence of 
methanol and $\mathrm{Et}_{3} \mathrm{~N}$ yielded methyl-4-methoxycubane-1carboxylate. 62

\subsubsection{Amide and (Hetero)arene-substituted Cubanes}

The incorporation of rigid units containing non-proteinogenic amino acids can introduce conformational restraints to the polypeptide structures. The specific conformation can increase molecular recognition at biological targets. A rigid linker-based amino acid synthesis was commenced from readily available 4(methoxycarbonyl)cubane-1-carboxylic (67) (Scheme 7). This monocarboxylic acid was subjected to a sodium borohydride $\left(\mathrm{NaBH}_{4}\right)$ catalyzed reduction and a pyridinium chlorochromate (PCC) catalyzed oxidation to access the methyl-4-formylcubane1-carboxylate (73). An asymmetric Strecker synthesis including the condensation of methyl-4-formylcubane-1-carboxylate (73) with $R-(-)-\alpha$-phenylglycinol followed by nucleophilic addition of trimethylsilyl cyanide (TMSCN) yielded major (2S) (74) derivative. Finally, (S)-2-(4'-carboxycubyl)glycine (76) was synthesized over two steps involving the conversion of the more abundant (2S)-acetonitrile (74) into aminoester (75), followed by hydrolysis. The overall yield of this synthesis was $14 \%$ with a $47 \%$ ee. ${ }^{63 a}$

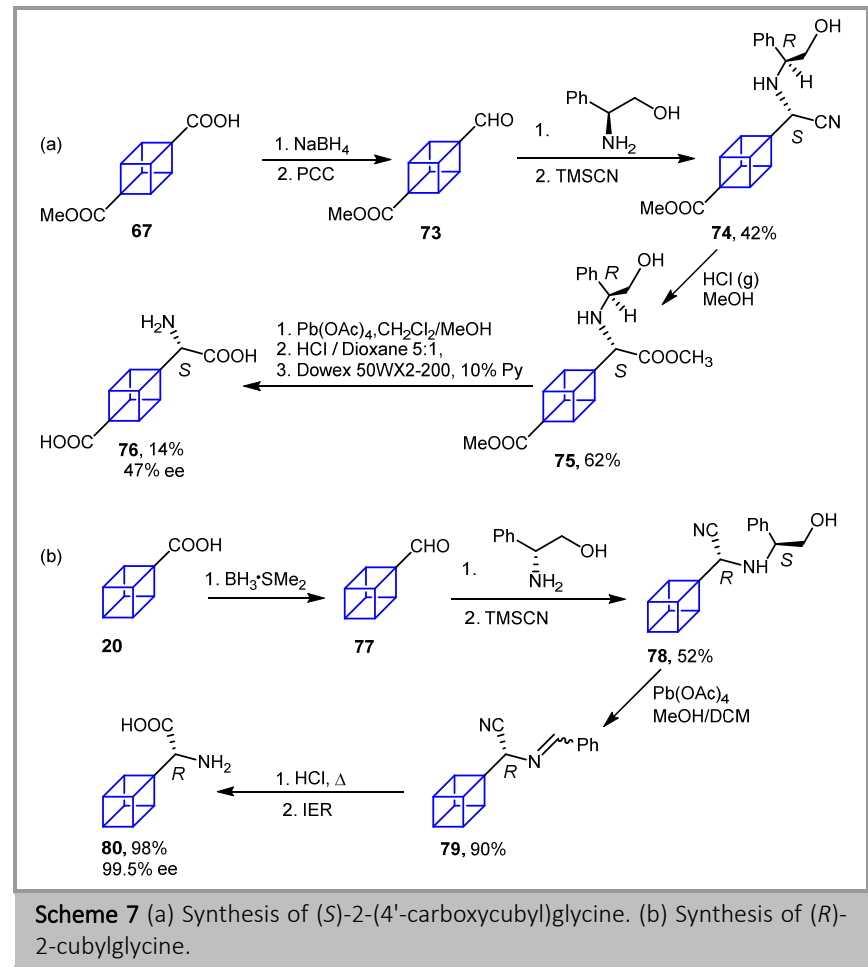

Moreover, other reports documented the synthesis of 2cubylglycin or its analog.63b Most recently, Williams and coworkers made an important contribution towards the synthesis of $(R)$-2-cubylglycine. 1-Formylcubane (77) was reacted with (S)-2-phenylglycinol and trimethylsilyl cyanide (TMSCN) under asymmetric Strecker reaction conditions. The major nitrile diastereomer (78) underwent lead(IV) acetate $\left(\mathrm{Pb}(\mathrm{OAc})_{4}\right)$ mediated oxidative cleavage followed by nitrile hydrolysis to deliver $(R)$-2-cubylglycin (80) in a $32 \%$ overall yield with $99.5 \%$ ee (Scheme 7). ${ }^{63 c}$
The relevance of (hetero)arene units in the pharmaceutical industry is evident; hence, (hetero)arene substituted cubanes were synthesized including pyridine, oxazole, triazole, pyrazole, and substituted cubanes ${ }^{5 a}$ A range of conventional synthetic methods were employed to afford these cubanes. In one example, pyridine substituted cubane was prepared using the Bohlmann-Rahtz reaction. Similarly, the 1-ethyl-3-(3dimethylaminopropyl)carbodiimide (EDC)-catalyzed amide coupling reaction of 1-amino-2-propanol with 4(methoxycarbonyl)cubane-1-carboxylic acid followed by a DessMartin oxidation afforded oxazole derivatives of cubane. ${ }^{53 b, 63 d}$

Recently, our research group reported the synthesis of bridgehead substituted cubanes using amide coupling reactions. This approach demonstrated a considerable substrate scope, from utilization of small aryl moieties (such as substituted phenyl ring) to large porphyrin rings. For e.g. the HATU/HOAtcatalyzed reactions of 4-iodoaniline with $\mathbf{6 7}$ and $\mathbf{9}$ yielded the 81 and 83 in $61 \%$ and $39 \%$ isolated yield respectively Additionally, we reported the synthesis of the very first amidebridged porphyrin-cubane-porphyrin arrays.63e The HATU/HOAt-catalyzed amide coupling of 5-(4'-aminophenyl)10,15,20-triphenylporphyrin with $\mathbf{6 7}$ and $\mathbf{9}$ afforded the 82 and 84 in $79 \%$ and $56 \%$ yield. A variety of cubane-porphyrin monomers and dimers with varying linker lengths and substituent angles were reported and can act as molecular tweezers.

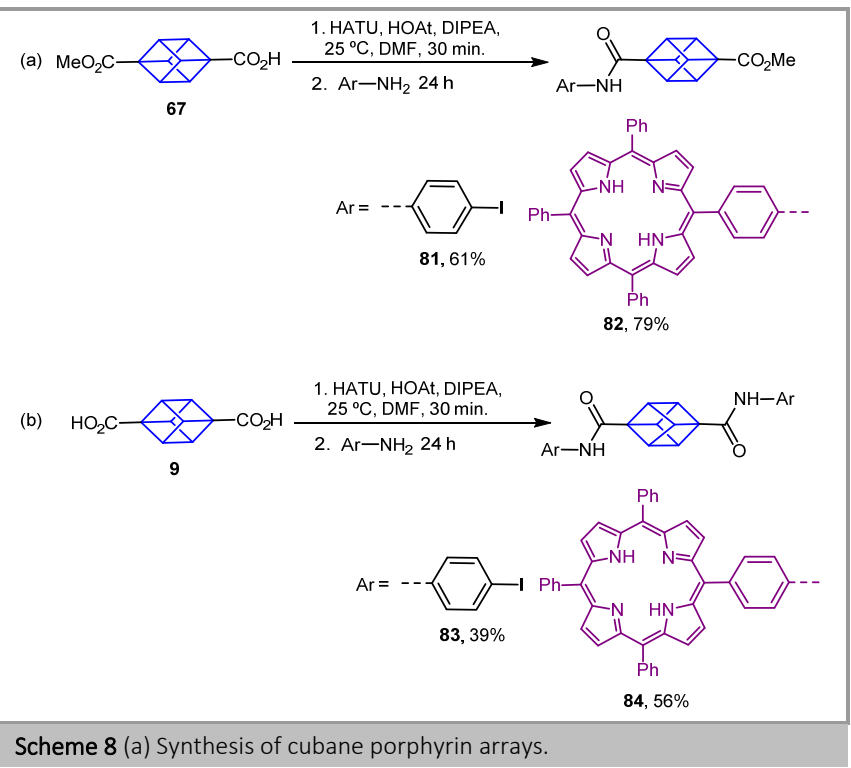

\subsubsection{Cubanes in Materials Science}

For decades, rigid scaffolds have been utilized in the delivery of drugs in the pharmaceutical industry. However, the potential of these scaffolds in material science is yet to be unlocked. In 2019, we gave an in-depth discussion on the material application of cubane derivatives. ${ }^{5 a}$ To highlight only some of the recent developments one area of interest is the use of cubanes in electron transfer systems and molecular circuitry. One of the basic requirements for efficient multi-chromophoric systems is the arrangement of chromophores in specific and inflexible orientations. Saturated rigid hydrocarbons allow the arrangement of bridgehead substituents at $180^{\circ}$. Moreover, saturated rigid linkers interrupt the undesired overlap of the $\pi$ - 
electronic system, thus, they are more desirable candidates for these systems compared to their conjugated analogs, i.e. benzene or ethynyl units. ${ }^{15 a}$ The cubane spacer enhances the rate of electron transfer by a factor of 10 when compared to cyclohexane. ${ }^{64}$ Interestingly, ab-initio calculations have shown that with increasing hydrocarbon skeleton strain, coupling between the two units taking part in the electron transfer (ET) is stronger. The calculated efficiency of ET was highest when the attached units were coplanar.65 Furthermore, the rate of electron transfer decreases exponentially as the distance between the units increased. Taking these facts into consideration, the most efficient system for ET should have two $\pi$-units attached via one rigid linker.

Cubane derivatives were also used in non-radiative singlet oxygen generating systems. ${ }^{66}$ The singlet oxygen was generated by $5,10,15,20$-tetraphenylporphyrin and pulse laser excitation. Different concentrations of cubane, methylcubane, cyanocubane, isopropanolcubane, phenylcubanone, and biphenylcubane were employed to record the decay of singlet oxygen. The biomolecular quenching rate constants were found to be between $10^{3}-10^{4} \mathrm{M}^{-1} \mathrm{~s}^{-1}$.

In both material and pharmaceutical applications, it is important to know intermolecular interactions within a specific environment. The three-dimensional geometry and highly symmetrical shape of cubane offers an intriguing possibility of supramolecular or non-covalent interactions. The development of facile synthetic procedures and the elucidation of cubanes' properties have encouraged researchers to investigate the supramolecular properties of this scaffold. The acidity of cubane $\mathrm{CH}$ is nearly equal to the phenyl $\mathrm{CH}^{32 \mathrm{a}}$ Therefore, both moieties have equal potential to interact with a hydrogen bond acceptor. ${ }^{32 \mathrm{~b}}$ While comparing the shapes of cubane and a phenyl ring, cubane offers two more potential sites for the interaction. Additionally, the angles of interactions differ from phenyl analog. Desiraju and coworkers reported non-covalent interactions in a series of 4-substituted cubane-1-carboxylic acids. It was observed that crystal structures of 4-halo-cubane1-carboxylic acids formed syn-anti catemers. ${ }^{32 \mathrm{~b}}$ Recently, our group discussed, in detail, non-covalent interactions in a variety of 1,4-substituted cubanes. ${ }^{32 c}$ The interactions between cubane $\mathrm{H}$-atoms and different functional groups such as amide, acid, ester, halogens, were investigated. Additionally, the noncovalent interaction profile of 1,4-substituted cubanes was compared with the 4-substituted benzoic acids. It was observed that crystal structures of 4-substituted cubane-1-carboxylic acids exhibit a 'mesh-like' hydrogen bond network. Catemer formation was found when a $\mathrm{CO}_{2} \mathrm{Me}$ group was introduced at the cubane skeleton. Halo-substituted cubanes exhibit halogenhydrogen interactions over hydrogen-hydrogen interaction. Complementarily, the most recent report on crystal structure of methyl-4-((4'-iodophenyl)carbamoyl)cubane-1-carboxylate $(\mathbf{8 1})^{32 \mathrm{c}}$ illustrated combined and repetitive intermolecular halogen and hydrogen bond interactions (Figure 2). The structure exhibits a head-to-tail $\mathrm{N} 1-\mathrm{H} \cdots \mathrm{O} 1=\mathrm{C}$ interaction at a distance of $2.853 \AA$ with an angle of $175.4^{\circ}$. The iodo-atom at the para-position of the phenylene moiety exhibits a head-to-tail halogen bond interaction with $02=\mathrm{C}$ of the ester group with a distance of $3.074 \AA$ and an angle of $170.1^{\circ}$. The observed noncovalent interactions are nearly orthogonal to each other. (a)
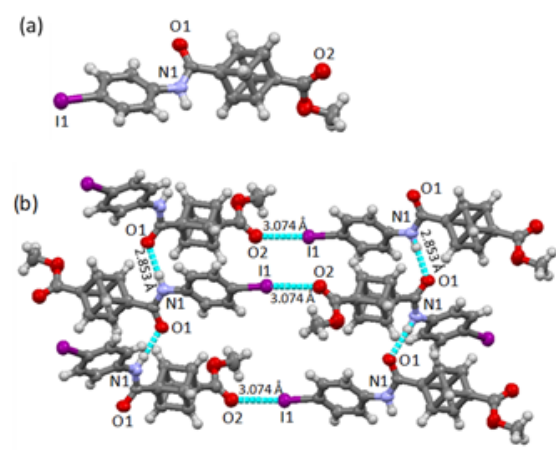

Figure 2. Non-covalent interactions in methyl-4-((4'iodophenyl)carbamoyl)cubane-1-carboxylate.

The specific 3D-orientation of cubane potentially favors this packing pattern enabling it to access this unusual iodine-oxygen interaction. Hence, this initial investigation into the interaction profile of cubanes provides a better understanding of substitution derived supramolecular pattern. Most recently, cubane-based MOFs were reported. A solvothermal reaction of cubane-1,4-dicarboxylic acid (9) and $\mathrm{Zn}\left(\mathrm{NO}_{3}\right)_{2} \cdot 6 \mathrm{H}_{2} \mathrm{O}$ led to colorless cubic crystals of the cubane-MOF. The higher number of $\mathrm{CH}_{2}$ moieties in the cubane-MOF led to increased absorption of $\mathrm{CO}_{2}$ and $\mathrm{CH}_{4}$ at lower pressures. ${ }^{32 \mathrm{~d}}$

\section{Bicyclo[1.1.1]pentane (BCP)}

\subsection{BCP Synthesis}

Strained, but remarkably stable, bicyclo[1.1.1]pentane (BCP, 2) is a unique hydrocarbon and exhibits an unusually short nonbonded distance between bridgehead carbons. ${ }^{67}$ The first BCP derivative was synthesized by Wiberg and his associates in 1964 via a ring closure reaction of 3-bromocyclobutane-1methylbromide (85) with sodium in dioxane (Scheme 9a; Route A). 68 Two years later, Wiberg and Connor reported the modified synthesis of bicyclo[1.1.1]pentane (2) and 1methylbicyclo[1.1.1]pentane. The reaction of lithium amalgam with 3-bromocyclobutane-1-methylbromide (85) in dioxane increased the yield up to 6\% (Scheme 9a; Route B). However, 1,4-pentadiene, a thermal isomer of BCP was identified as the major product $(94 \%)$ in this reaction. 69

Meinwald and coworkers documented the gas phase photolysis of bicyclo[2.1.1] hexane-2-one (86) to access the BCP (2) skeleton. Even though the yield of bicyclo[1.1.1lpentane (16$20 \%$ ) was considerably improved over that obtained in the procedures described above, the main drawback of the method is the requirement for decarbonylation to be effected in the gasphase, thus limiting the scale of operation (Scheme 9a; Route C). Unfortunately, in the condensed phase, ring-opened products were found to predominate.70 In another approach, 2,2dichloro-3-(methoxycarbonyl)bicyclo[1.1.1]pentane-1-

carboxylic acid (89) was synthesized using the addition of dichlorocarbene $\left(\mathrm{Cl}_{2} \mathrm{C}\right.$ :) to methyl 3-phenylbicyclobutane-1carboxylate (86) (Scheme 9b). ${ }^{71}$ Although this procedure has limited substrate scope, for several years it was the only method employed in the synthesis of bridged and bridgehead substituted BCPs. In further attempts to provide alternative methods, it was reported that the synthesis of BCP skeleton 
could be achieved from its more strained relative [1.1.1]propellane (92).

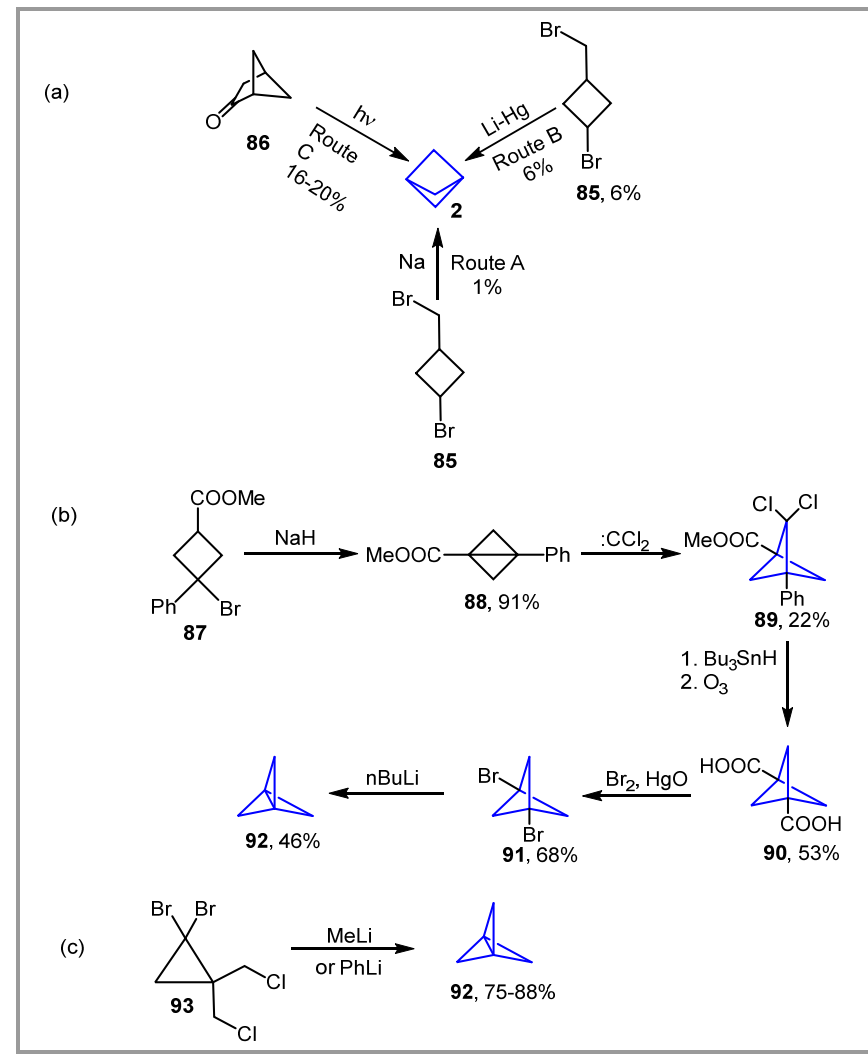

Scheme 9 (a) Synthesis of bicyclo[1.1.1]pentane. (b) Synthesis of [1.1.1]propellane. (c) Contemporary synthesis of [1.1.1]propellane.

Eighteen years after the first report on BCP synthesis, Wiberg et al. reported the synthesis of [1.1.1]propellane (92) via reaction of 1,3-dibromobicyclo[1.1.1]pentane (91) with $n$-BuLi.72 Reaction of sodium hydride with methyl 3-bromo-3phenylcyclobutane-1-carboxylate (87) gave methyl 3phenylbicyclo[1.1.0]butane-1-carboxylate (88) in 91\% yield. Addition of dichlorocarbene to 3-phenylbicyclo[1.1.0]butane-1carboxylate (88) access the methyl-2,2-dichloro-3phenylbicyclo[1.1.1]pentane-1-carboxylate (89). The latter compound was subjected to dechlorination to yield bicyclo[1.1.1]pentane-1,3-dicarboxylic acid (90). The key synthetic steps involve the Hunsdiecker reaction followed by reaction with $n$-BuLi to afford [1.1.1]propellane (92) (Scheme 9b). Szeimies and associates invented a facile path to access the [1.1.1]propellane (92) in two steps. First, the addition of dibromocarbene to 3-chloro-2-chloromethylpropene gives the 1,1-dibromo-2,2-bis(chloromethyl)cyclopropane (93), in a next step further treatment of this stable tetrahalide (93) with alkyl lithium gives the [1.1.1]propellane (92).73a An improved procedure was also reported including the improved yields of the tetrahalide, which is commercially available (Scheme 9c). ${ }^{73 b, 73 c}$

Different assumptions have been made about the nature of the central bond in [1.1.1]propellane (92). Initial computational studies revealed that the central bond has a negative orbital overlap. $^{74 a}$ In 1983, Wiberg and coworkers suggested that a single bond exists between bridgehead carbons ( $\left.65 \mathrm{kcal} \mathrm{mol}^{-1}\right)$ which is more stable than the corresponding diradical. ${ }^{74 b}$
However, crystal structure analysis of [3.1.1]propellane concluded the absence of electron density along the central axis. $^{74 c}$ Further investigation towards these contradictory results led to the assumption that the central bond is a $\sigma$ bridged $\pi$-bond, which is similar to bonding in boranes/carboranes. ${ }^{74 \mathrm{~d}}$ Earlier NMR studies to characterize the hybridization concluded that the central bond has more $s$ character. In contrast, a recent NMR investigation revealed the central bond to have more $\pi$-character. ${ }^{74 \mathrm{e}}$ Thus, due to the contradictory experimental and theoretical data, the true nature of central bonds in [1.1.1]propellane (92) remains a mystery. A more recent investigation by $\mathrm{Wu}$ and associates suggested that the C1-C3 bond is a charge shift bond. ${ }^{74 \mathrm{f}}$

\subsection{BCP Functionalization}

In 1990, Weiberg and Waddell demonstrated the free radical addition of alkyl/aryl halides, thiols, and iodine to [1.1.1]propellane (92), which granted access to a wide variety of 1,3-disubstituted BCP derivatives. However, in some cases, free radical addition led to oligomerization of the parent hydrocarbon. ${ }^{75}$ The bond between the two bridgehead carbons in [1.1.1]propellane (92) is quite reactive towards radical addition. Hence, the most common approach to synthesize bridgehead substituted BCPs are radical reactions on [1.1.1]propellane (92). The development of this methodology was the most significant breakthrough in BCP chemistry over the past five and a half decades. ${ }^{73 a}$ The development of synthetic protocols promoted the industrial-scale production of 1,3disubstituted bicyclo[1.1.1]pentane. For example, SpiroChem in Switzerland conducts the kilo gram-scale synthesis of BCP derivatives.

\subsection{1 lodo-BCPs and their Transformations}

The reaction of $I_{2}$ with [1.1.1]propellane (92) yielded the 1,3bisiodobicyclo[1.1.1]pentane (94). Similarly, reacting organoiodide with [1.1.1]propellane (92) generates the 1-iodo3-substitutedbicyclo[1.1.1]pentane (95). Conceptually, the thus generated (BCP)C-I bond can be dissociated in three ways: (i) by generating the bicyclo[1.1.1]pentylcation via solvolysis of the bridgehead halide (Scheme 10; Route A), (ii) by the bicyclo[1.1.1]pentylanion that can be generated via metalhalogen exchange (Scheme 10; Route B), and (iii) by photo- or chemically induced homolytic cleavage of the C-X bond to generate the bicyclo[1.1.1]pentane radical (Scheme 10; Route C).

Bicyclo[1.1.1]pentylcations (96) are not stable intermediates and undergo rearrangement to bicyclo[1.1.0]butyl-1-carbinyl cations (97). These unstable cations react further with solvents or nucleophiles via bond migration to form 3methylenecyclobutyl-1-derivatives (98) (Scheme 10; Route A). ${ }^{76}$ In contrast to 1-halo substituted BCPs, 1,3bisiodobicyclo[1.1.1]pentane (94) is stable towards solvolysis. However, it does react with sodium ethoxide in methanol and reverts back to [1.1.1]propellane (92) via $\mathrm{I}_{2}$ elimination. After further investigation, it was observed that the reaction of 1,3bisiodobicyclo[1.1.1]pentane (94) with methanolic $\mathrm{KOH}$ afforded 1-iodo-3-methoxy-bicyclo[1.1.1]pentane (102). Wiberg and McMurdie proposed that the reaction preceded over two steps. The first step involved the in situ generation of $\mathrm{CH}_{3} \mathrm{OI}$ and 
[1.1.1]propellane (92). The next step involved the reaction of these two intermediates to access the 1-iodo-3-methoxybicyclo[1.1.1]pentane (102) and the 1iodobicyclo[1.1.1]pentylcation was trapped with azide ions.76b Hence, this method offered the first route to 3-iodobicyclo[1.1.1]pentyl-1-azide (103). Later, Timberlake and Hossain documented the synthesis of 3-iodobicyclo[1.1.1]pentyl-1-azide (103), which was achieved using the addition of iodine azide $\left(\mathrm{IN}_{3}\right)$ to [1.1.1]propellane (92)(Scheme 10b). ${ }^{77}$
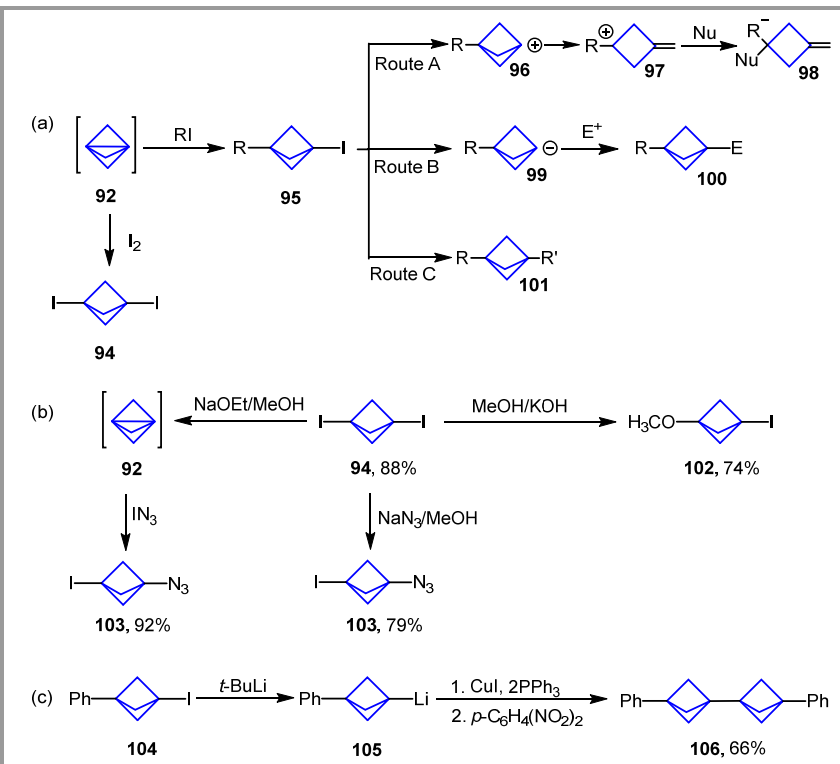

(d)
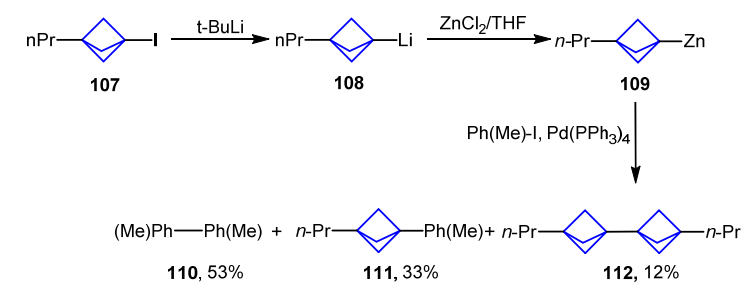

(e)

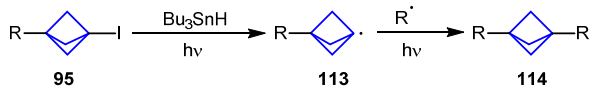

Scheme 10 Synthesis of iodo-BCPs and subsequent reactivity with electrophiles, nucleophiles and radical initiators.

Metal-halogen exchange reactions are a facile way to generate the bicyclo[1.1.1]pentylanion (Scheme 10a; Route B). The reaction of 1 -(phenylthio)bicyclo[1.1.1]pentane with lithium 4,4'-di-tert-butylbiphenyl gave 1-lithiumbicyclo[1.1.1]pentane. 1-iodobicyclo[1.1.1]pentane reacts with $t$-BuLi to produce 1lithiumbicyclo[1.1.1]pentane. Interestingly, the success of reactions with organolithium reagents depends upon the nature of the halogen as 1-chlorobicyclo[1.1.1]pentane is relatively inert. ${ }^{78,76 a}$ 1-Lithiumbicyclo[1.1.1]pentane reacts with a variety of electrophiles as explored by Wiberg and coworkers. ${ }^{78} \mathrm{Michl}$ and coworkers reported the synthesis of [n]staffanes via homo coupling of 1-lithiumbicyclo[1.1.1]pentane (Scheme 10c). The relative stability of bridgehead carbanions facilitates the in situ preparation of transition metal (Pd or Ni) complexes of BCPs at low temperatures. In retrospect, one caveat of this procedure is the low yields of the desired products. Upon further investigation, the coupling reaction of 1-lithium-3- phenylbicyclo[1.1.1]pentane (105) with 1,4-dinitrobenzene using the copper(I) iodide-triphenylphosphine (CuI- $\left.\mathrm{PPh}_{3}\right)(1: 2)$ complex was comparatively successful and the desired 3,3'diphenyl[2]staffane (106) was achieved. ${ }^{79}$

In most of the documented syntheses, iodo derivatives of BCP were used without further purification but the use of crude reaction mixtures is not fruitful for coupling reactions. To remove this troublesome step, de Meijere and coworkers ${ }^{80}$ developed an elegant pathway to synthesize 1,3-disubstituted BCPs $(111,112)$. Halogen lithium exchange was followed by transmetallation with anhydrous $\mathrm{ZnCl}_{2}$ and it furnished a variety of bicyclo[1.1.1]pentyl-1-zinc derivatives (109) (Scheme $10 \mathrm{~d})$. These organozinc complexes were coupled with a variety of aryl and alkenyl halides using a variety of catalysts including 1,2-bis(diphenylphosphino)ethane]nickel(II) chloride (Ni(dppe) $\left.\mathrm{Cl}_{2}\right)$, palladium tetrakis(triphenylphosphine) $\left(\mathrm{Pd}\left(\mathrm{PPh}_{3}\right)_{4}\right)_{1}$ and

$\left[1,1^{\prime}-\right.$

bis(diphenylphosphino)ferrocene]dichloropalladium(II)

$\left(\mathrm{Pd}(\mathrm{dppf}) \mathrm{Cl}_{2}\right)$. This was the first report on use of transition metal catalysts in coupling rections of BCPs but under specified conditions a mixture of products $(\mathbf{1 1 0}, 111$ and 112) was observed.

The tributyltin hydride $\left(n \mathrm{Bu}_{3} \mathrm{SnH}\right)$ catalyzed reduction of $\mathbf{9 5}$ is a mild way to generate $\mathrm{C}-\mathrm{C}$ bonds at the bridgehead position of BCPs. The reaction of $n \mathrm{Bu}_{3} \mathrm{SnH}$ with 95 generates the bicyclo[1.1.1]pent-1-yl radical (113), which can be trapped (Scheme 10e). The simplest example involves the photochemical trapping of bicyclo[1.1.1]pent-1-yl radical with $\mathrm{H} \cdot$. Additionally, unsaturated olefins (e.g., styrene) and unsaturated esters react well with 95 in the presence of $n \mathrm{Bu}_{3} \mathrm{SnH}$ and light. As discussed above, R-I ( $\mathrm{R}$ = alkyl, aryl) addition to [1.1.1]propellane (92) relies on the use of mercury lamp irradiation or the implementation of a highly reactive halide, for example, $\mathrm{CF}_{3} \mathrm{I}$.

In an alternative approach, triethyl borane (BEt $\left.t_{3}\right)$ was utilized as a radical initiator, which promotes atom transfer radical addition (ATRA) of organohalides to [1.1.1]propellane (Scheme 11). ${ }^{81 a}$ This methodology was further extended to synthesize the nucleoside (117), dipeptide (120), and fentanyl analog of BCP (123). The reaction of [1.1.1]propellane (92) with $\alpha$ iodooxazolidinones (124) provided access to 1-iodo-3oxazolidinone substituted BCPs. Further treatment of these derivatives with tris(trimethylsilyl)silane (TTMS) gave the BCP oxazolidinones (125), which proved to be a key compound for the generation of stereogenic centers adjacent to the BCP skeleton. The $\alpha$-chiral-bicyclo[1.1.1]pentane derivatives (126) were obtained via diastereoselective asymmetric enolate functionalization of BCP oxazolidinones (125). The hydrolytic cleavage of oxazolidinone $\mathbf{1 2 6}$ accesses the $\mathbf{1 2 7}$ in 93\% yield. The BCP analog of phenylglycine (128) was synthesized via deprotection of the tert-butyloxycarbonyl (Boc) group and Ptcatalyzed hydrogenation of $\mathbf{1 2 7} .{ }^{81 \mathrm{~b}}$ 


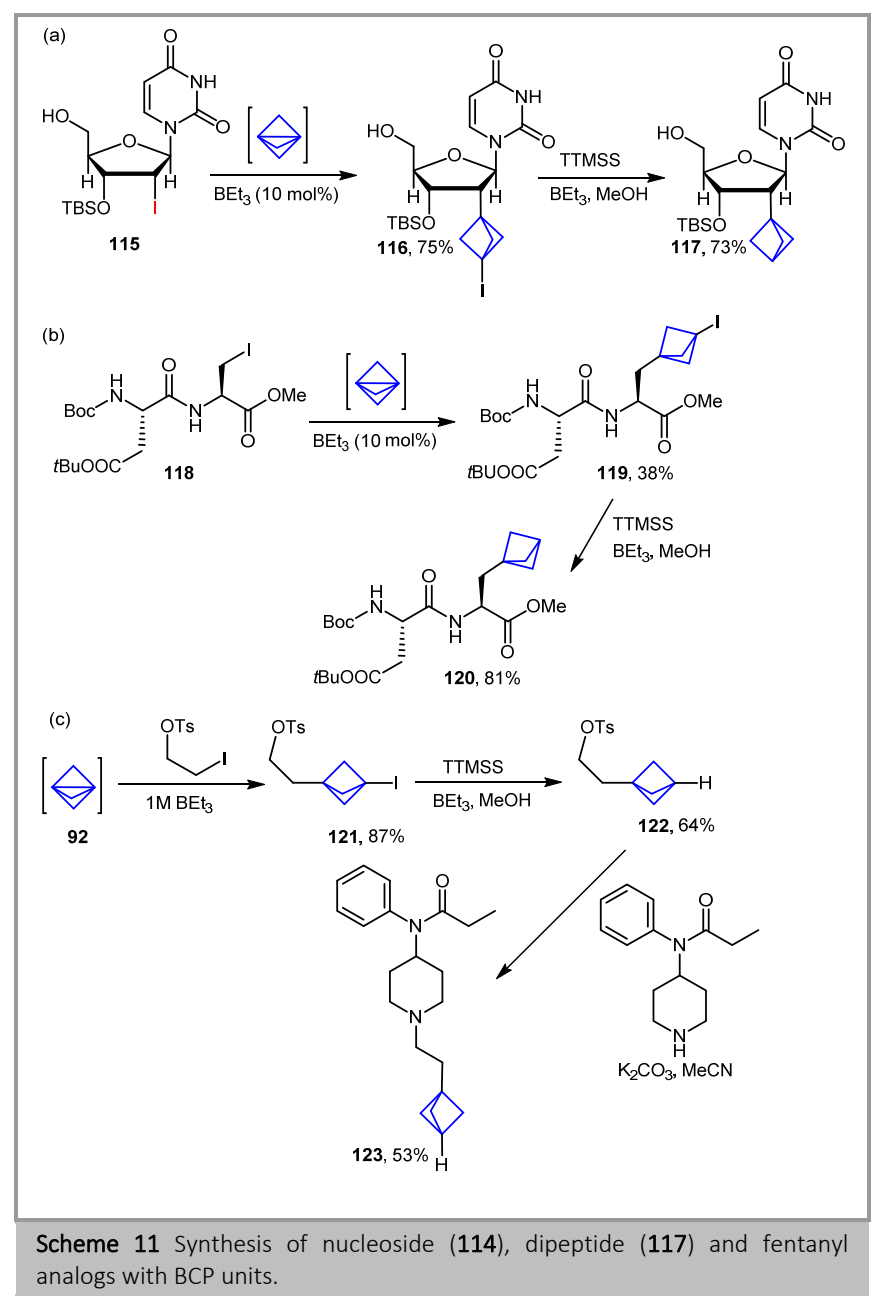

This procedure is highly efficient for alkyl halides but it has limited functional group tolerance and could not provide access to $\mathrm{C}_{\mathrm{sp} 3}-\mathrm{C}_{\mathrm{sp} 2}$ bonds. ${ }^{81}$ Recently, Anderson and coworkers reported that these restrictions could be overcome by photocatalyzed atom transfer radical addition (ATRA) reactions. ${ }^{81 c}$ The reaction of [1.1.1] propellane (92) with organohalide in the presence of the $\quad f a c$-tris(2-phenylpyridinato)iridium(III) $\quad\left(f a c-\operatorname{Ir}(\mathrm{ppy})_{3}\right)$ photocatalyst and blue LEDs led to the formation of 1-iodo-3substituted BCPs (129). These reaction conditions were tolerated by a variety of substrates including (hetero)aryl, $1^{\circ} / 2^{\circ}$ alkyl, (hetero)benzylic, and $\alpha$-enolates derivatives (Scheme 12b). Importantly, the BCP-penicillin G analog (132) was synthesized using the aforementioned strategy. Light-Ir dual catalyzed addition of $\mathbf{1 3 0}$ to [1.1.1]propellane yielded $\mathbf{1 3 1}$ in an isolated yield of $78 \%$. In the next step, synthesis of penicillin G surrogate of BCP (132) was achieved by deiodination of 131 .

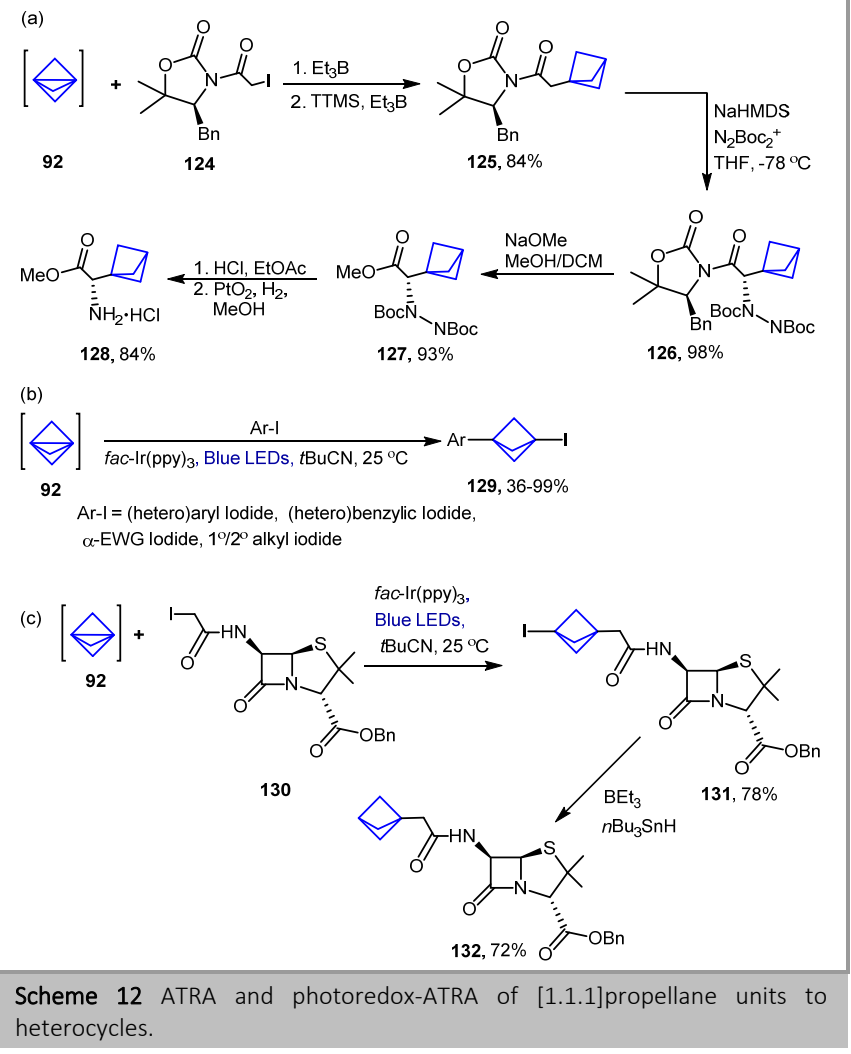

\subsubsection{Alkynyl-substituted BCPs}

The combination of acetylene subunits and rigid scaffolds leads to the synthesis of interesting rigid molecular systems due to their linear geometry. ${ }^{82}$ The 1,3-bisethynylbicyclo[1.1.1]pentane (135) was used in the construction of the molecular building blocks $^{82 a}$ (Scheme 13 and 14). Photoaddition of freshly distilled bisacetyl to [1.1.1]propellane (92) easily afforded 1,3diacetylbicyclo[1.1.1]pentane (133), which was further converted into 1,3-bisethynylbicyclo[1.1.1]pentane 135 in two steps. Treatment of 1,3-diacetylbicyclo[1.1.1]pentane (133) with triphenylphosphine/ hexachloroethane in refluxing benzene afforded 1,3-bis(1-chlorovinyl)bicyclo[1.1.1]pentane (134). In the next step, reaction of $\mathrm{NaNH}_{2} / \mathrm{NH}_{3}$ with chlorovinylbicyclo[1.1.1]pentane (134) produced the 1,3diethynylbicyclo[1.1.1]pentane (135) in $80 \%$ yield.82a To prevent oligomerization, the ethynyl groups were protected with trimethylsilyl (TMS) groups (Scheme 13a). In contrast to the parent 1,3-bisethynylbicyclo[1.1.1]pentane (135), the more stable mono- and di-silyl derivatives $(136,137)$ can be stored at low temperatures for a few months. Note, $\mathbf{1 3 5}$ is an explosive in the crystalline state.

1,3-Bisethynylbicyclo[1.1.1]pentane (135) can be used as a nonconjugative alternative to traditional $\pi$-conjugative linkers. ${ }^{83}$ Kaleta et al. reported modified synthesis of $\mathbf{1 3 5}$ and developed the synthetic methods to introduce 135 into larger structures (Scheme 13b).83b The applicability of $\mathbf{1 3 5}$ in Sonogashira coupling reactions has been widely reported in the literature. ${ }^{82,83} \mathbf{1 3 5}$ is integral to the synthesis of shape persistent macrocycles (SPMs). 


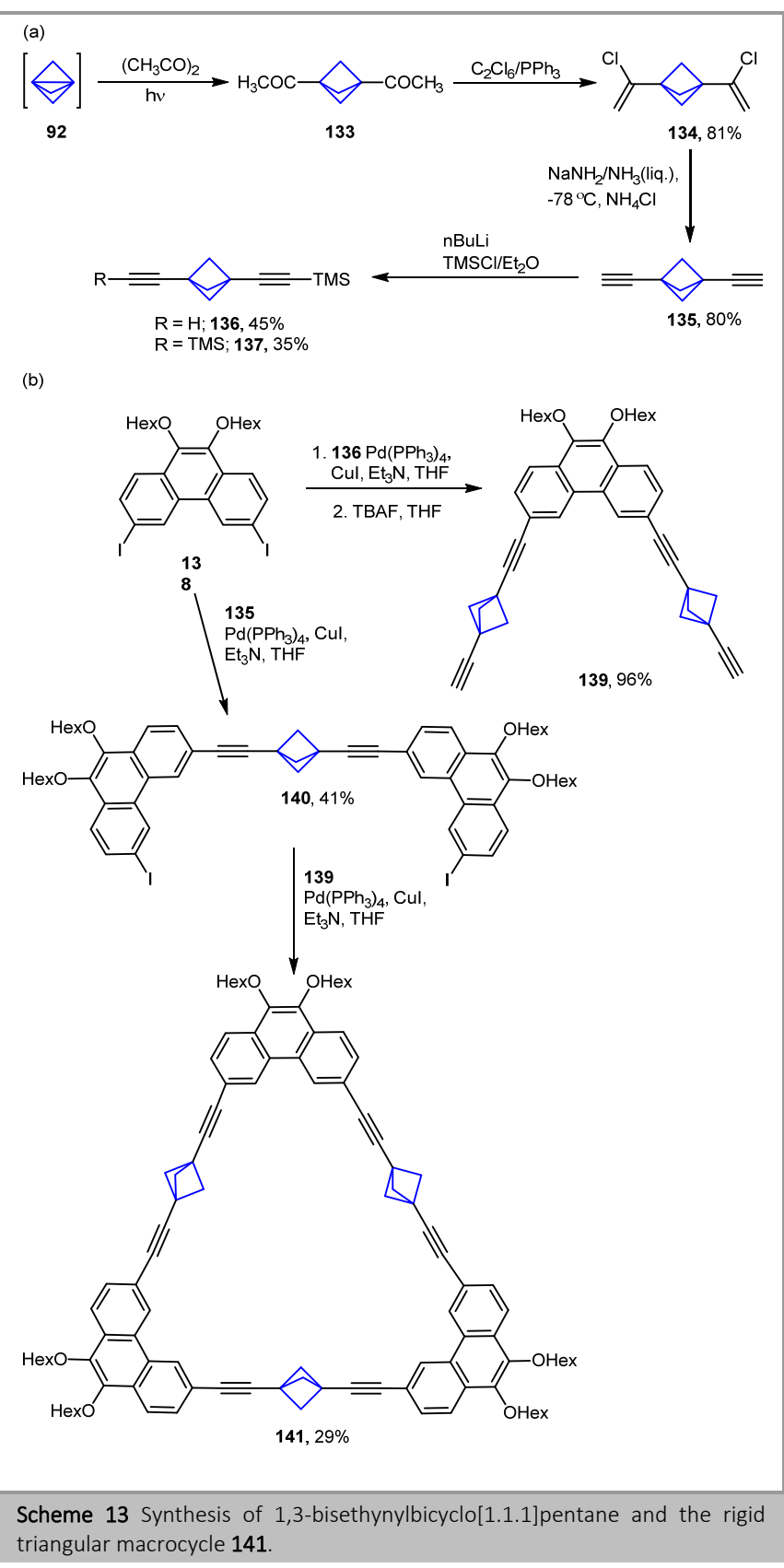

The Sonagashira coupling between 136 and 138 yielded the Vshaped 3,6-bis((3"-ethynylbicyclo[1.1.1]pentan-1'yl)ethynyl)phenanthrene-9,10-diyl dihexanoate (139). The coupling reaction of $\mathbf{1 3 5}$ with excess 3,6-diiodophenanthrene9,10-diyl dihexanoate (138) afforded the rod-like rigid molecule 140. The synthesis of triangular macrocycle 141 was achieved via standard Sonogashira coupling reactions between V-shaped 139 and rigid rod like 140 (Scheme 13b). ${ }^{83 \mathrm{~b}}$

Michl and coworkers utilized $\mathbf{1 3 6}$ as a key intermediate in a Cadiot-Chodkiewicz coupling to synthesize rigid rod molecules (Scheme 14a). The reaction of $\mathbf{1 3 6}$ with $n$-BuLi followed by $\mathrm{CuCl}$ generated the asymmetric dimer 143 in an isolated yield of 54\%. Further deprotection of 143 and subsequent Sonogashira reaction with 4-bromopyridine hydrochloride provided the pyridine-terminated molecular rod $\mathbf{1 4 4}$ in a $74 \%$ isolated yield (Scheme 14a). ${ }^{83 \mathrm{~d}}$ In general, the synthetic utility of alkynes can be extended by their Umpolung to 1-haloalkynes as they are key compounds that undergo several chemical transformations
(Scheme 14b). 1,3-Bis(bromoethynyl)bicyclo[1.1.1]pentane (145) was synthesized in a $81 \%$ yield using NBS in the presence of silver nitrate.

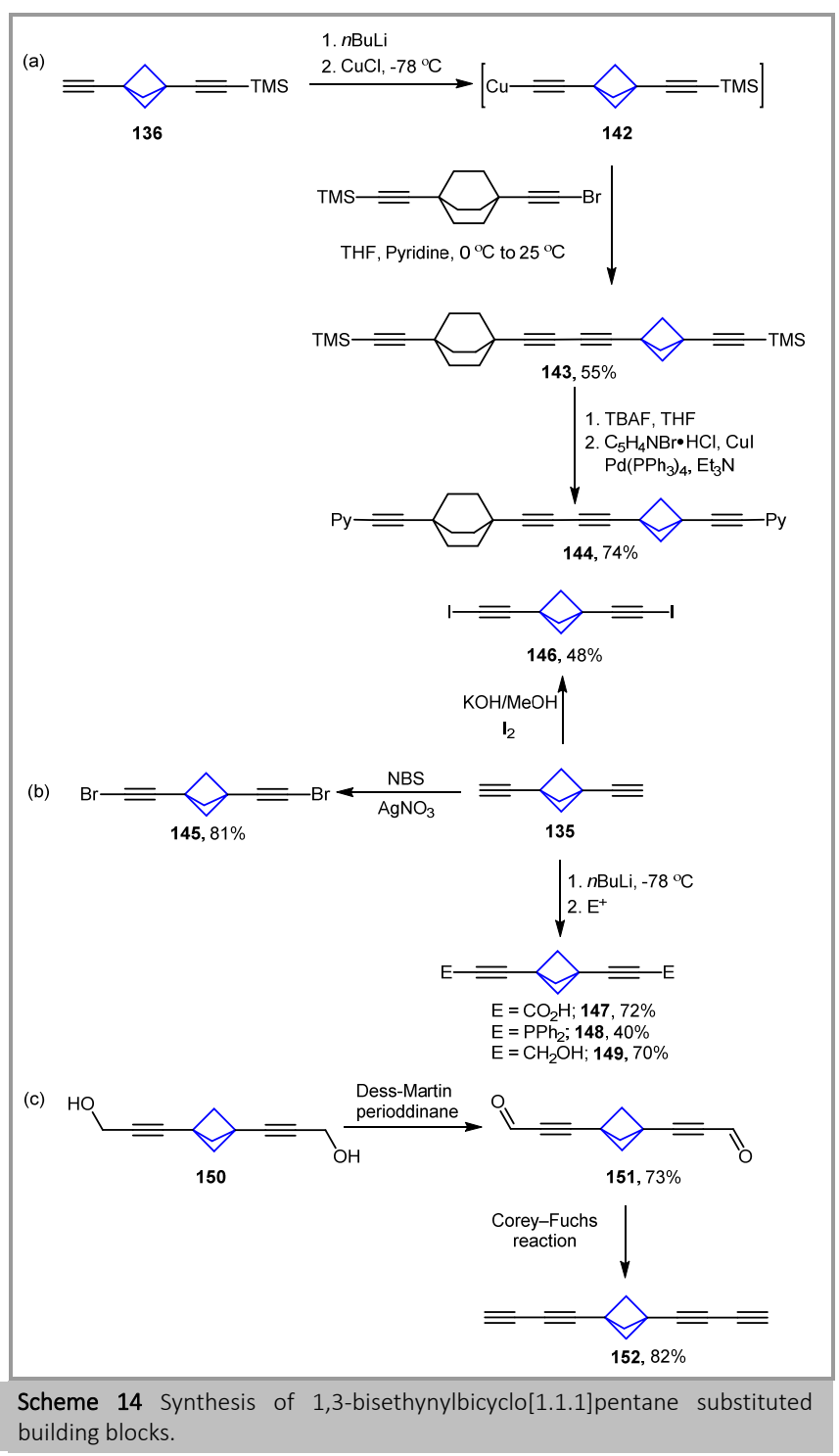

In another approach, 1,3-bis(iodoethynyl)bicyclo[1.1.1]pentane (146) was synthesized by reaction of the alkyne with $I_{2}$ and $\mathrm{KOH}$ in $\mathrm{MeOH}$ (Scheme 14b). The reaction of lithium acetylides of 135 with various electrophiles including carbon dioxide $\left(\mathrm{CO}_{2}\right)$, chlorodiphenylphosphine $\left(\mathrm{PPh}_{2} \mathrm{Cl}\right), \quad$ and paraformaldehyde $\left(\left(\mathrm{CH}_{2} \mathrm{O}\right)_{\mathrm{n}}\right)$ afforded 1,3-bis(propiolic acid)bicyclo[1.1.1]pentane (147), 1,3bis(phosphane)bicyclo[1.1.1]pentane (148), and 1,3-bis(prop2'-yn-1'-ol)bicyclo[1.1.1]pentane (149). 1,3-Bis(prop-2'-yn-1'ol)bicyclo[1.1.1]pentane (149) was employed as an entry point in the Corey-Fuchs synthesis for the stepwise construction of additional triple bonds (Scheme 14c). The reaction of 149 with a Dess-Martin periodinane afforded 3,3'-(bicyclo[1.1.1]pentane1,3-diyl)dipropiolaldehyde (150). In the final step, 150 was subjected to a Corey-Fuchs reaction to give 1,3-di(buta-1', $3^{\prime}$ diyn-1'-yl)bicyclo[1.1.1]pentane (151) (Scheme 14c). ${ }^{84}$ Similar to 135,148 was successfully subjected to coupling reactions for further derivatization. The ethynyl building blocks $(\mathbf{1 3 5}, \mathbf{1 3 6}$, 
and 137) of BCP have been successfully introduced into molecular rods, rotors, connectors, and orthogonal rods. ${ }^{85}$

\subsubsection{Transition Metal-catalyzed Coupling Reactions}

In contrast to the cubane skeleton, the BCP scaffold is more tolerant towards conventional 'Pd-catalyzed' coupling reactions. For example, the reaction of alkyl/aryl Grignard reagents with [1.1.1]propellane (92) facilitates the formation of 3-substituted bicyclo[1.1.1]pentyl-1-magnesium halide derivatives (153). Coupling reactions (Kumada and Negishi) with 3-substituted bicyclo[1.1.1]pentyl-1-magnesium derivatives were utilized to afford the 1,3-bis-substituted BCPs (155). Reactions with Grignard reagents are often dependent upon the choice of solvents and temperature. Szeimies and coworkers reported the $\mathrm{Pd}(\mathrm{dppf}) \mathrm{Cl}_{2}$ catalyzed coupling reaction of 3substitutedbicyclo[1.1.1]pent-1-yl magnesium bromide with bromoarenes (Scheme 15a; Route A). ${ }^{86 a}$ Akin to cross-couplings, $\left[\mathrm{Pd}\left(\mathrm{CH}_{3} \mathrm{CN}\right)_{2}\right] \mathrm{Cl}_{2} / \mathrm{MeBr}$ catalyzed homo-coupling reactions of 3 substitutedbicyclo[1.1.1]pentyl-1-magnesium halides proceeded readily and led to the formation of 1,1' bis(bicyclo[1.1.1]pentane) or [2]staffanes derivatives (154). (Scheme 15a; Route B).86b

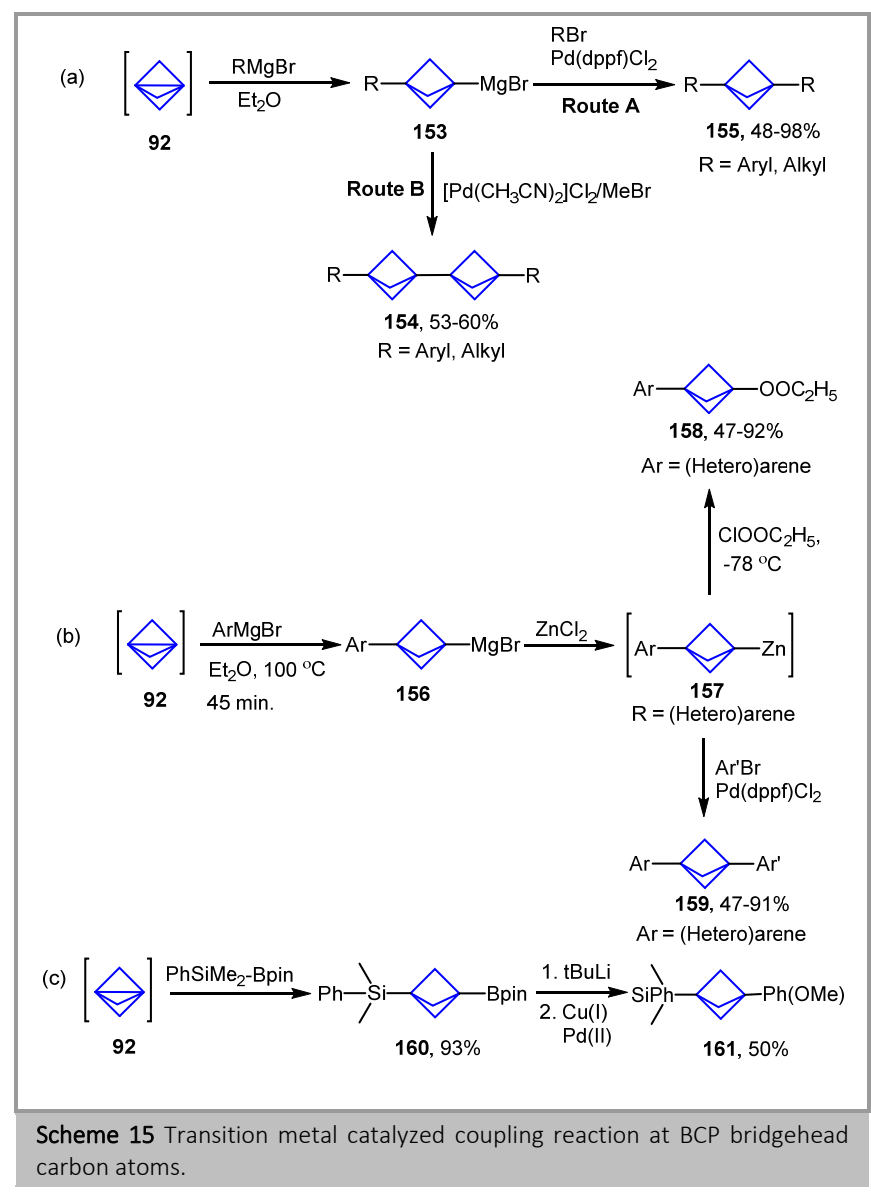

As described above, Kumada coupling reactions were first attempted to access 1,3-bissubstituted BCPs. However, the long reaction time, formation of a complex mixture of products, and variable yields impeded access to these derivatives. The BCP Grignard reagents are comparatively stable and transmetallation on BCP magnesium halides with zinc chloride generates BCP zinc chloride derivatives (157). $\mathrm{PdCl}_{2}(\mathrm{dppf})$ catalyzed coupling reactions between bicyclo[1.1.1]pentylzinc chlorides and aryl halides are a powerful tool for the construction of 1,3-bis-(hetero)arenebicyclo[1.1.1]pentanes (159). In pioneering work Knochel and coworkers ${ }^{86 c}$ described a modified route for the preparation of bisarylated BCPs. The addition of aryl magnesium bromide to [1.1.1]propellane, followed by heating at $100{ }^{\circ} \mathrm{C}(45 \mathrm{~min}-3 \mathrm{~h})$ resulted in improved yields (up to 92\%) of the 3-(hetero)arenebicyclo[1.1.1] pentyl-1magnesium derivatives (156). Transmetallation with $\mathrm{ZnCl}_{2}$ and Negishi cross-coupling reactions resulted in a variety of pharmaceutical analogs. Moreover, the reaction of 3(hetero)arenebicyclo[1.1.1]pentyl-1-zinc derivatives (157) with ethyl chloroformate gave the corresponding esters (158). These addition reactions proved to be very convenient and provided a broader substrate scope than earlier reports. Silaboration of [1.1.1]propellane (92) with silyl substituted pinacolborane (PhSiMe 2 -Bpin) proceeded smoothly to yield 1dimethylphenylsilane-3-(pinacolborane)-bicyclo[1.1.1]pentane (156). The latter was reacted with $t$-BuLi, followed by a $\mathrm{Cu}_{2} \mathrm{O} / \mathrm{Pd}(\mathrm{dppf})_{2} \mathrm{Cl}_{2}$ catalyzed cross-coupling reaction with 4bromoanisole to access a $\mathrm{C}_{\mathrm{sp}}{ }^{2}-\mathrm{C}_{\mathrm{sp}}{ }^{3}$ bond at the inert bridgehead alkane (160) (Scheme 15c).86c

Most recently, it was observed that three or four-component reactions of [1.1.1]propellane (92) with Grignard reagents, boronic esters, and electrophiles proceeded efficiently to afford 1,3-disubstituted BCPs. ${ }^{86 \mathrm{~d}}$ It was also shown that treating 1substituted bicyclo[1.1.1]pentyl magnesium bromide and vinylbpin with the catalyst LiCl formed the boronate complex of BCP. Subsequently, $\mathrm{I}_{2}$ and NaOMe facilitated the simultaneous 1, 2migration and boron elimination to yield 1,3-bisfunctionalized BCPs (Scheme 16a). BCP Grignard 162 was easily accessed from reaction of 2-tolylmagnesiumbromide and 92 . Further $\mathrm{LiCl}$ catalyzed addition of 2-propenylboronic ester to $\mathbf{1 6 2}$ formed BCP-boronate 163, which was subsequently reacted with iodine and sodium methoxide to facilitate 1,2-migration and borone elimination, yielded alkenyl-BCP 164 in 89\% yield. Similar to theses synthetic efforts, adamantan-1-yl-(4,5,6,7-tetrachloro1,3-dioxo-1,3-dihydro-isoindol-2yl)-acetate rhodium(II $\left(\mathrm{Rh}_{2} \text { (TCPTAD) }\right)_{4}$ catalyzed intermolecular donor/acceptor diazo insertion at the tertiary position of BCPs was achieved. A reaction of 3-(4'-trimethylsilane)phenylbicyclo[1.1.1]pentane (165) with 2,2,2-trichloroethyl 2-diazo-2-phenylacetate proceeded smoothly in presence of ( $\left.\mathrm{Rh}_{2}(\mathrm{TCPTAD})_{4}\right)$. Importantly, this procedure provides $\mathrm{BCP}$ derivative (166) in $91 \%$ yield in $91 \%$ ee. 86 e

Conversely, transition metal-catalyzed coupling reactions on [1.1.1]propellane (92) led to the formation of cyclobutane derivatives followed by ring strain release. The reaction of [1.1.1]propellane (92) with $\mathrm{Rh}(\mathrm{I})$ or $\mathrm{Pd}(\mathrm{II})$ occur instantaneously and the ring-opened dimer formed, however, in the presence of $\operatorname{Ag}(\mathrm{I}), \operatorname{Ir}(\mathrm{I}), \operatorname{Pt}(\mathrm{II})$ or $\mathrm{Pt}(0)$ various dimeric and trimeric products were formed. ${ }^{75}$ Most of the aforementioned reactions proceeded via a metal carbene intermediate. A reaction of [1.1.1]propellane (92) with terminal alkynes in the presence of $\mathrm{CuI}$ and $\mathrm{N}, \mathrm{N}$-diisopropylethylamine (DIPEA) yielded exocyclic allenic cyclobutanes.87a For example, $\mathrm{Cu}$-catalyzed addition of [1.1.1]propellane (92) to phenylpropargyl ether provide access to ( $3-(3)$ - 
methylenecyclobutylidene)allyl)oxy)benzene (167) (Scheme 16c). Recently, Aggarwal and coworkers achieved the synthesis of spiro compound $\mathbf{1 6 8}$ by the nickel-catalyzed reaction of styrene with [1.1.1]propellane (92). ${ }^{87 \mathrm{~b}}$

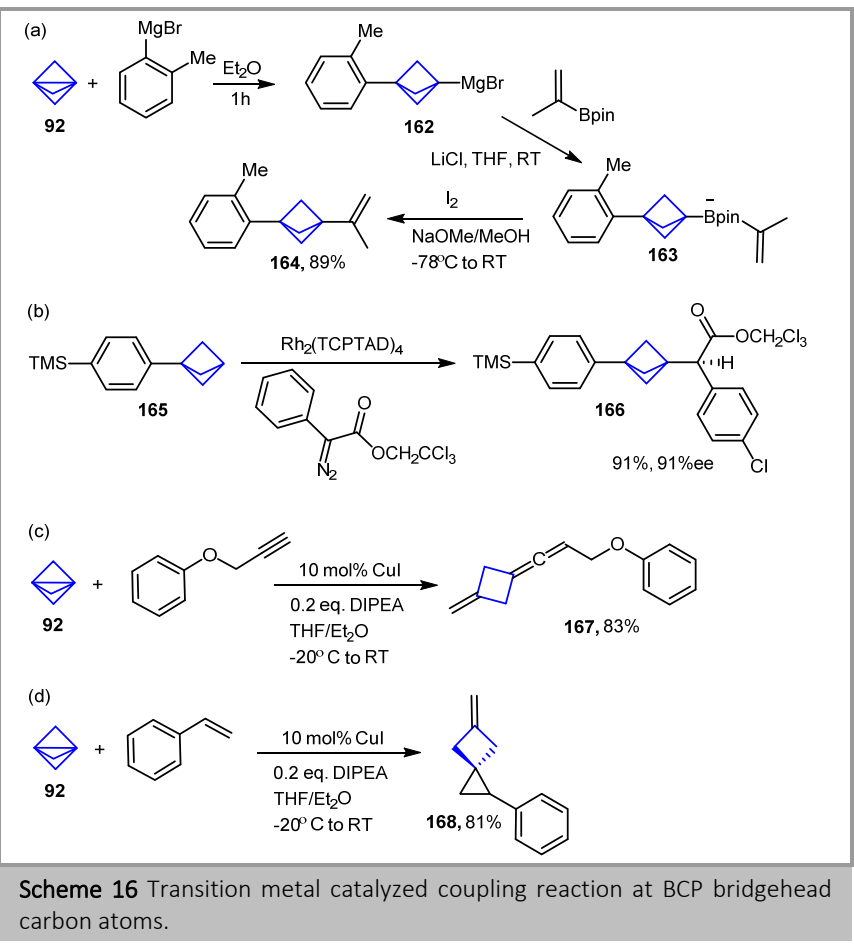

\subsubsection{N-substituted BCPs}

The utilization of BCP as part of a potential drug candidate is growing. Considering the significance of $\mathrm{N}$-containing moieties in medicinal chemistry, synthetic access to BCP amines is of utmost interest. Here, Wiberg and coworkers employed the Schmidt reaction for the conversion of bicyclo[1.1.1]pentane-1carboxylic acid (169) to BCP-1-amine hydrochloride (170) (Scheme 17a) ${ }^{88 a}$ and in an attempt to improve the protocol, a Hofmann rearrangement was applied.88b Barbachyn and coworkers exploited the electrophilic attack of lithium methoxyamine on bicyclo[1.1.1]pentan-1-yl lithium (171) to access bicyclo[1.1.1]pentane-1-amine. However, the neutral amine was not isolated; instead the benzamide analog 161 formed in a $24 \%$ yield (Scheme 17b). ${ }^{88 c}$ Due to poor yields, the above mentioned procedures did not provide a practical solution to the problem. Then, Bunker and associates from Pfizer reported the synthesis of 1-bicyclo[1.1.1]pentylhydrazine over two steps from [1.1.1]propellane and further reduction of hydrazine gave the desired bicyclo[1.1.1]pentane-1-amine hydrochloride (170) (Scheme 17c). ${ }^{88 d}$ Along the same line, efforts were made to synthesize 3-phenylbicyclo[1.1.1]pentane1-amine hydrochloride (173) via the reduction of 1-azido-3iodobicyclo[1.1.1]pentane (103) (Scheme 17d). ${ }^{88 e, f}$ Additionally, 1-azido-3-iodobicyclo[1.1.1]pentane (103) was also used for the click reaction with alkynes derivatives. ${ }^{88 f}$ Notably, Baran and coworkers, reported the addition of a broad range of "turbo amides" to [1.1.1]propellane to access monoamine BCPs (174) (Scheme 17e).89a,b This strain release strategy diversified the range of substrates with a multitude of desirable bioisosteres. It allows for large synthesis and has been applied to the deca-gram scale synthesis of bicyclo[1.1.1]pentane-1-amine hydrochloride (170).

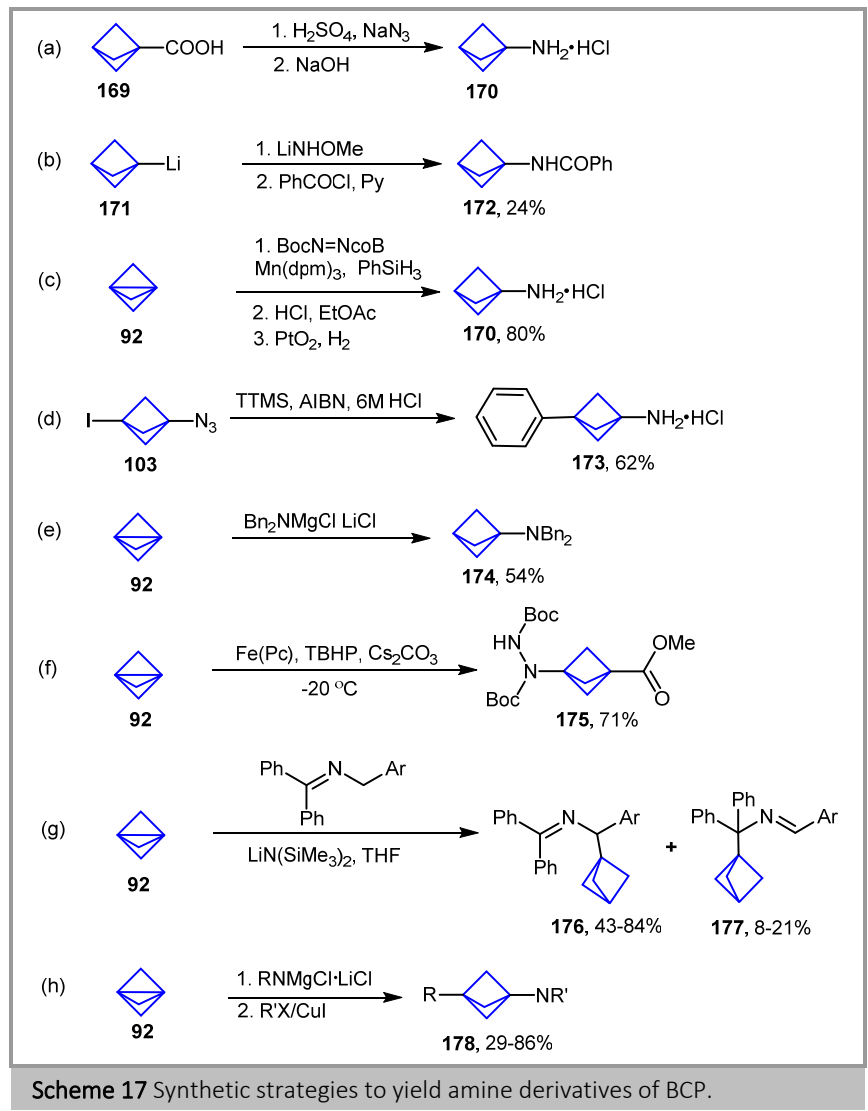

While this strategy solved the long-standing challenge of integrating BCP as a terminal group, this approach did not access the highly desirable 3-substituted bicyclo[1.1.1]pentane1 -amine $(\mathbf{1 7 5}, \mathbf{1 7 8})$. Kanazawa et al. reported the radical multicomponent carboamination of [1.1.1]propellane to access 1,3-disubstituted bicyclo[1.1.1]pentane. ${ }^{89 \mathrm{c}}$ The Iron phthalocyanine (Fe(Pc)) catalyzed carboamination of [1.1.1]propellane (92) with hydrazyl reagents (radical precursor) and di-tert-butyl azodicarboxylate (radical acceptor) gave the di-tert-butyl 1-(3'(methoxycarbonyl)bicyclo[1.1.1]pentan-1'-yl)hydrazine-1,2dicarboxylate (175) (Scheme 17f). This method is a rare example of a highly controlled one-pot di-functionalization. The deprotonation of $\mathrm{N}$-benzyl ketamines generates 2-azaallyl anions that exhibit super electron-donating (SED) character. The reaction of $N$-benzyl ketamines with [1.1.1]propellane (92) in the presence of two eq. of lithium bis(trimethylsilyl)amide proceeded smoothly at room temperature to yield BCP benzylamines (176 and 177) (Scheme 17g).89d Recently, Gleason and coworkers reported the synthesis of a 3alkylbicyclo[1.1.1]pentan-1-amine (178) from [1.1.1]propellane over two steps. The first step involves the addition of a turbo amide, which generates the metallated intermediate. In the next step, that intermediate is further reacted with an electrophile in the presence of CuI (Scheme 17h). ${ }^{89 \mathrm{e}}$

The amide bond is omnipresent in biologically and pharmaceutically active compounds. Its semi-rigid nature and H-bonding ability promotes the formation supramolecular architectures. To this end, our group recently reported the 
synthesis of porphyrin-BCP-porphyrin arrays. The reaction of 4iodoaniline with 3-(methoxycarbonyl)bicyclo[1.1.1]pentane-1carboxylic acid (179) and BCP-1,3-dicarboxylic acid (90) and yielded the methyl-3- $\left((4)^{\prime}-\right.$ iodophenyl)carbamoyl)bicyclo[1.1.1] pentane-1-carboxylate (180) and $N^{1}, N^{3}$-bis(4'-iodophenyl)bicyclo[1.1.1]pentane1,3dicarboxamide (181) respectively (Scheme 18). BCP derivatives 180 and 181 were subjected to standard 'Pdcatalyzed coupling reactions. In one of example, Suzuki crosscoupling reactions between dicarboxamide derivatives 181 and porphyrins 182 yielded a porphyrin-BCP-porphyrin array 183 in $60 \%$ isolated yield (Scheme 18b).63e

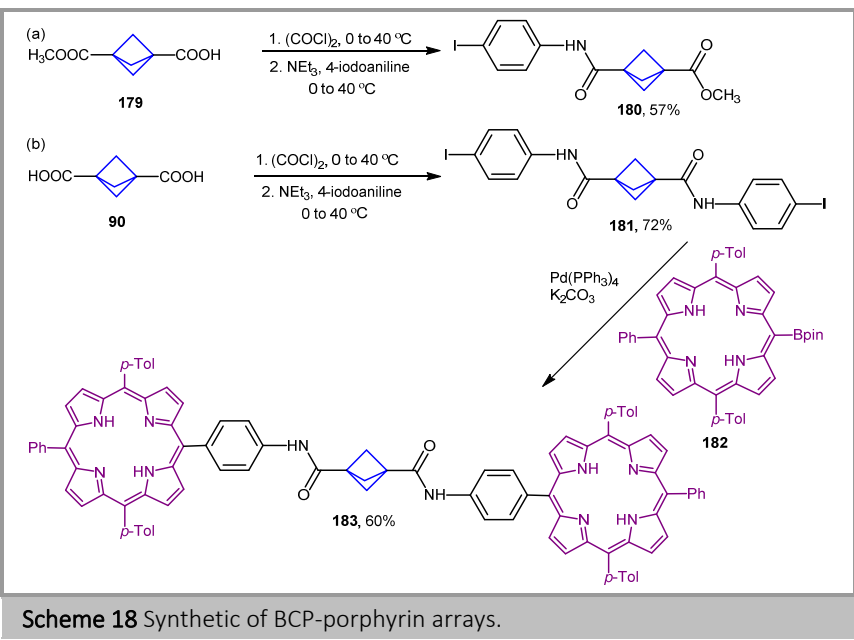

\subsubsection{SET Reactions}

Over the last decade, the development of SET-based crosscoupling reactions involving BCP-NHPIs $(\mathbf{1 8 4}, \mathbf{1 8 5})$ under thermal or photolytic conditions led to a library of diverse compounds with C-X (X = C, N B, Si, Se, S) bonds. ${ }^{18}$ Aggarwal and coworkers reported decarboxylative borylation, which involved the illumination of a BCP-NHPI (184) under visible light at room temperature in the presence of bis(catecholato)diboron $\left(\mathrm{B}_{2} \mathrm{Cat}_{2}\right)^{61}$ Baran and coworkers reported the enantioselective transformation of redox-active esters into amino acids. E.g., the reaction of a BCP-NHPI with a glyoxylate-derived chiral acceptor ethyl 2-((mesitylsulfinyl)imino)acetate in the presence of $\mathrm{Zn}$ (3 eq.) and $\mathrm{Ni}(\mathrm{OAc})_{2} \cdot 4 \mathrm{H}_{2} \mathrm{O}$ yielded the $\mathrm{BCP}$ derivative 182 (Scheme 19a). ${ }^{90 a}$

Similarly, Liang et al. discovered that $\mathrm{Cu}$-photoredox catalyzed decarboxylative coupling generates a $\mathrm{CSP}_{\mathrm{S}}{ }^{3} \mathrm{~N}$ bond at bridgehead carbons. Photochemical treatment of the readily available 3methoxycarbonylbicyclo[1.1.1]pentane-1-carboxylic acid (189) with indole in the presence of Cu catalyst 191 and an Ir-catalyst 192 afforded the N-substituted BCP 193 (Scheme 19b).90b Importantly, this procedure is versatile and applicable to a variety of $\mathrm{N}$-nucleophiles including nitrogen heterocycles, amides, sulfonamides, and anilines.

Appending boronic esters or acids at the bridgehead carbon is of utmost interest due to their utility in Suzuki-Miyaura coupling reactions. Disappointingly, bpin-substituted BCPs do not react under standard Suzuki-coupling conditions. However, the BCP$\mathrm{BF}_{3} \mathrm{~K}$ complex has been utilized as an entry point for metallaphotoredox coupling reactions. The reaction of bicyclo[1.1.1]pentane-1-carboxylic acid (169) or bicyclo[1.1.1]pentane-1-trifluoroborate salt (194) with a (hetero)arene (195) in the presence of a Ni/Ir dual catalyst enables the formation of heteroarene-BCP complexes (193) (Scheme 19c)..$^{90 \mathrm{c}}$

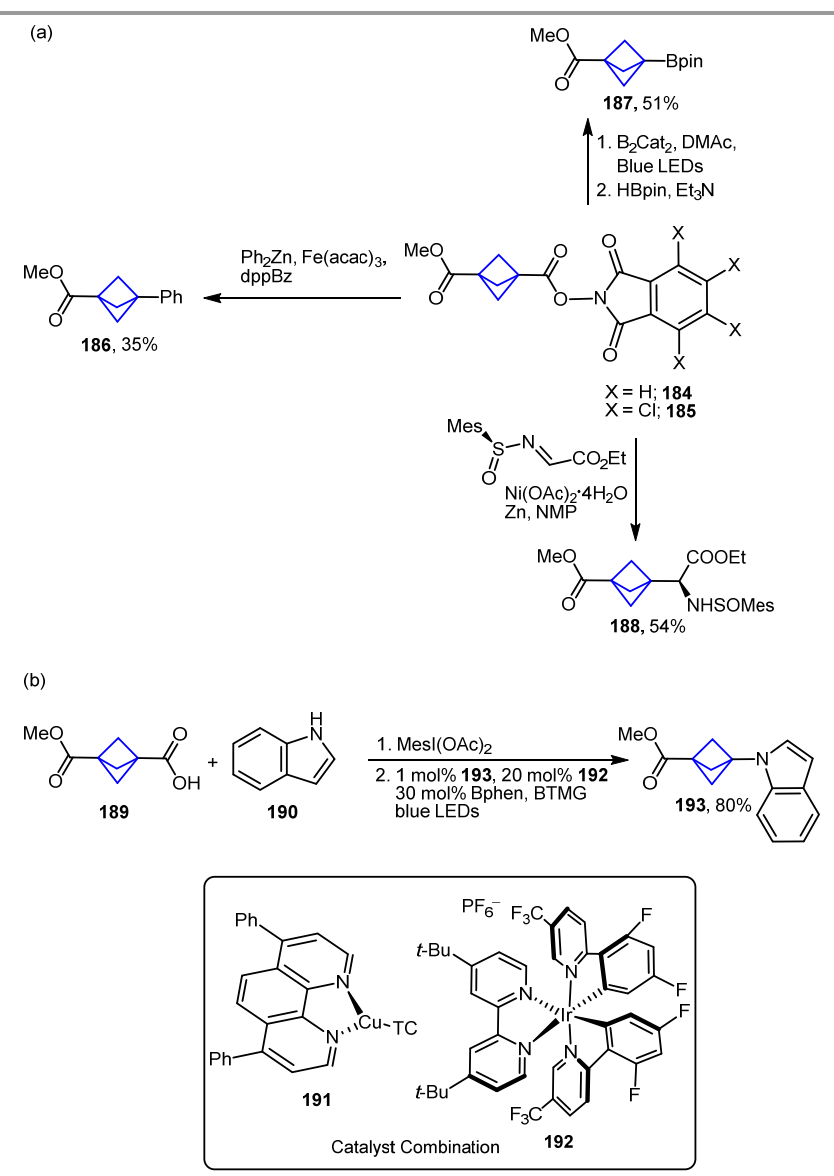

(c)

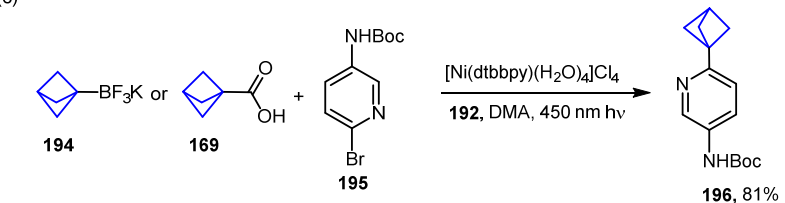

(d)

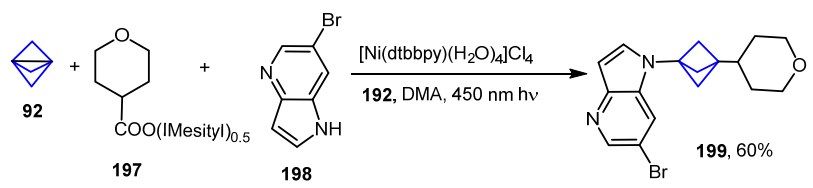

Scheme 19 Redox cross-coupling at BCP skeleton.

As discussed earlier, multicomponent reactions are an important tool to access amine substituted BCPs. This approach is effective and allows for the formation of complex BCP structures in one step. Recently, MacMillan and associates reported the three-component photoredox radical coupling of [1.1.1]propellane (92) and various $N$-, $P$ - and $S$-nucleophiles. Given the susceptibility of [1.1.1]propellane (92) to a variety of organic radicals, photoredox-derived alkyl radicals react with [1.1.1]propellane (92) to give BCP radicals. Thereafter, the in situ reaction of the $\mathrm{BCP}$ radical with a copper complex of a nucleophile gives a 1,3-bis-substituted BCP. For example, an 
Ir/Cu-catalyzed one-pot three-component reaction with 1.2 eq of [1.1.1]propellane (92) and tetrahydropyran 197 generated the BCP (1.0 eq) of the N-nucleophile 198 to afford BCP derivative 199 (Scheme 19d).90d In parallel attempts, lightdriven radical strain release amino functionalization was reported as an alternative strategy to get access BCP-amino derivatives. ${ }^{90 \mathrm{e}}$

\subsubsection{Thio-substituted BCPs}

Several elegant methods have evolved to generate radical intermediates that give access to a variety of BCP products. The radical addition of [1.1.1]propellane (92) to sulfides and thiols was reported by Wiberg and coworkers. ${ }^{69}$ However, a systematic study and substrate scope was not reported. Recently, the Bräse laboratory documented the insertion of [1.1.1]propellane (92) into aromatic sulfides. These reactions proceeded smoothly under ultraviolet light at room temperature. The reaction conditions are quite tolerant towards a number of substrates allowing for halo-, hydroxy-, methoxy-, carboxy-, amino-, and nitro-substituted thiols to be appended to [1.1.1]propellane (92). ${ }^{91}$ The thiol derivatives of BCP (200) were synthesized by addition of [1.1.1]propellane (92) to aromatic thiols. Additionally, 200 was oxidized to 201 and 202 using peracid oxidation (Scheme 20a). In subsequent efforts, BCP-sulfoximines (203) were synthesized from BCP-sulphides (200). The synthesis of BCP sulfoximine commenced from the reaction of $\mathbf{2 0 0}$ with bis(trifluoroacetoxy)iodo]benzene (PIFA; oxidizing agent) and ammonium carbonate ( $\mathrm{NH}$ source). However, this reaction did not proceed well with electronwithdrawing or sterically demanding substituents. Additionally, copper(I)-catalyzed $N$-arylations with aryl halides were performed to diversify the scope of the products (Scheme 20a). ${ }^{91 c}$

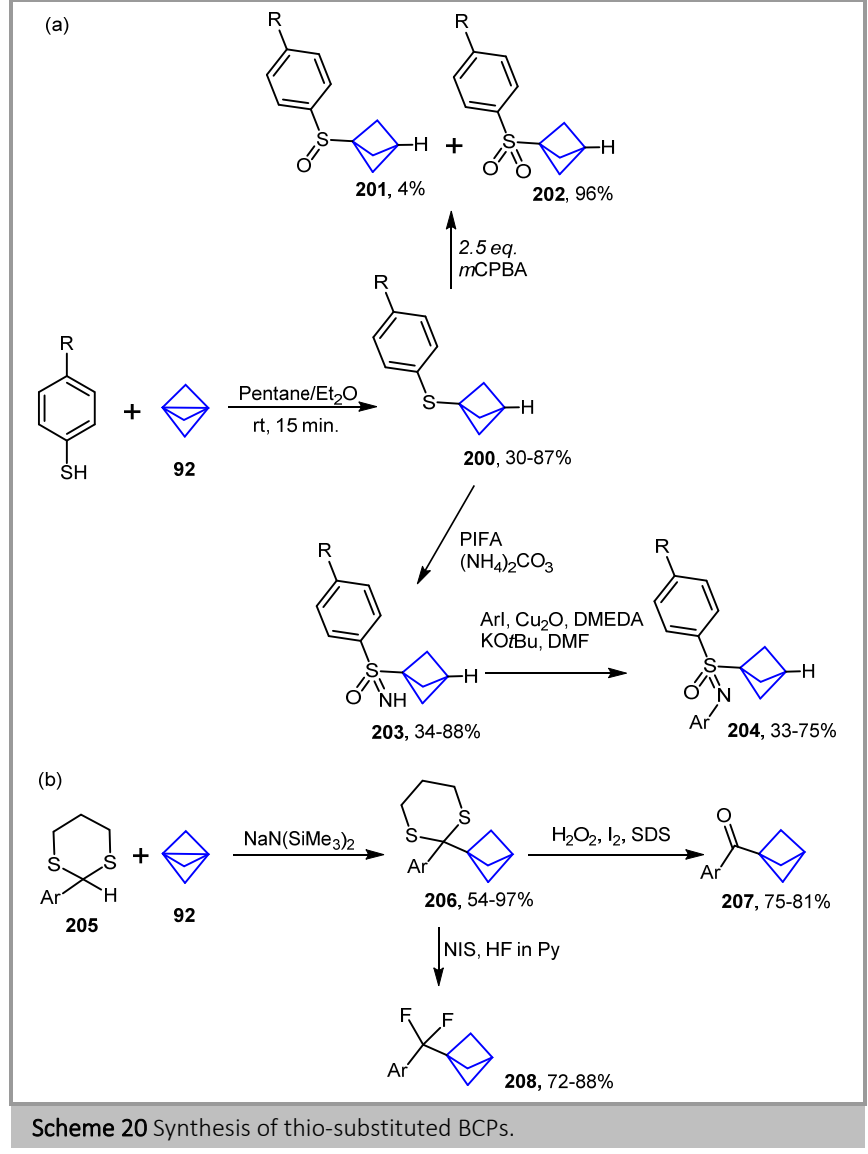

Dithianes are bench stable building blocks in synthetic organic chemistry and more often serve as a starting point for successive functionalization. In 1965, Corey and Seebach reported the addition of electrophiles to metallated dithianes. A similar synthetic strategy was adopted to access the diarylketone analogs of BCP. The addition of 2-aryl-1,3dithianes (pronucleophile) (205) to [1.1.1]propellane (92) in the presence of sodium bis(trimethylsilyl)amide $\left(\mathrm{NaN}\left(\mathrm{SiMe}_{3}\right)_{2}\right)$ yielded the 2-(bicyclo[1.1.1]pentan-1-yl)-2-aryl-1,3-dithiane (206). Further hydrolysis of the dithane compound yielded the aryl keto derivative of the BCP (207) (Scheme 20b).92a Complementary to the above-mentioned studies, successful radical addition of [1.1.1]propellane (92) to xanthate has been achieved as well. ${ }^{92 \mathrm{~b}}$

All the synthetic studies described acknowledge the great difficulties associated with the integration of BCP unit/s into complex structures. Despite these synthetic difficulties, the BCP unit was incorporated between a switchable photochromatic unit (1,2-bis(thiophen-3-yl)hexafluorocyclopentene) and a fluorophore (anthracene). ${ }^{93}$

\subsubsection{Bridge-substituted BCPs}

Even though radical addition reactions involving [1.1.1]propellane have been widely exploited, bridge(methylene)-substituted BCP could not be obtained through this method. The starting material employed in the synthesis of propellane and the highly reactive nature of the C1C3 bond limits the insertion of functionalities at the bridge positions. Hence, derivatization at the bridge positions is not a trivial task. Michl and associates reported the synthesis of 2,2diflurosubstituted bicyclo[1.1.1]pentane-1,3-dicarboxylic acids. 
By bubbling $F_{2}$ gas through a solution of dimethyl bicyclo[1.1.1]pentane-1,3-dicarboxylate (209) in 1,1,2trichlorotrifluoroethane,

dimethyl-2,2,4,4,5,5hexafluorobicyclo[1.1.1]pentane-1,3-dicarboxylate (210) was obtained. Although this was the first report of bridge fluorination, the use of $F_{2}$ gas, the need for specialized glassware, and the tedious separation of polyfluorinated derivatives limits the implementation of this procedure (Scheme 21a). ${ }^{94 a, b}$

Kaleta et al. then reported the synthesis of bridge-chlorinated bicyclo[1.1.1]pentane-1,3-dicarboxylic acids; however, this reaction is also quite sensitive towards reagents and temperature. ${ }^{94 c}$ Another logical synthetic pathway to achieve bridge substituted BCP derivatives is the addition of carbene to the bicyclo[1.1.0]butane moiety. This pathway is comparatively unexplored and overlooked as most researchers focus on $\mathrm{CH}$ activation, i.e. functionalization at bridgehead carbons.

Bridge-substituted BCPs were employed in the synthesis of lipoprotein-associated phospholipase A2 (LpPLA2) inhibitor, which was developed for the treatment of atherosclerosis. In one example, the replacement of BCP within LpPLA2 inhibitor involved the addition of a carbene derivative to a bicyclo[1.1.0]butane, which was followed by a dechlorination reaction. 3-oxocyclobutane-1-carboxylic acid (211) was transformed into methyl 3-(4'(trifluoromethyl)phenyl)bicyclo[1.1.0]butane-1-carboxylate (212) over four synthetic steps. The key synthetic step involved the reaction of sodium trichloroacetate $\left(\mathrm{CCl}_{3} \mathrm{COONa}\right)$ in tetrachloroethane to afford methyl-2,2-dichloro-3-(4'(trifluoromethyl)phenyl)bicyclo[1.1.1]pentane-1-carboxylate (213) (Scheme 21b). The latter compound underwent a standard amide transformation, followed by a reduction to afford the LpPLA2 analog.95a. Recently, Ma et al. reported the synthesis of 2,2-difluorobicyclo[1.1.1]pentanes (215) via difluorocarbene insertion into a bicyclo[1.1.0]butane derivative (214). The key reagent, $\mathrm{CF}_{2}$-carbene, was generated in situ from a trimethylsilyl 2-fluorosulfonyl-2,2-difluoroacetate/sodium fluoride (TFDA/NaF) mixture (Scheme 21c). ${ }^{95 b}$ Similarly, the reaction of trifluoromethyltrimethylsilane/sodium iodide ( $\left.\mathrm{CF}_{3} \mathrm{TMS} / \mathrm{NaI}\right)$ with aryl/vinylsubstituted bicyclo[1.1.0]butanes afforded 2,2'-difluorosubstituted BCPs (Scheme 21d). ${ }^{95 c}$

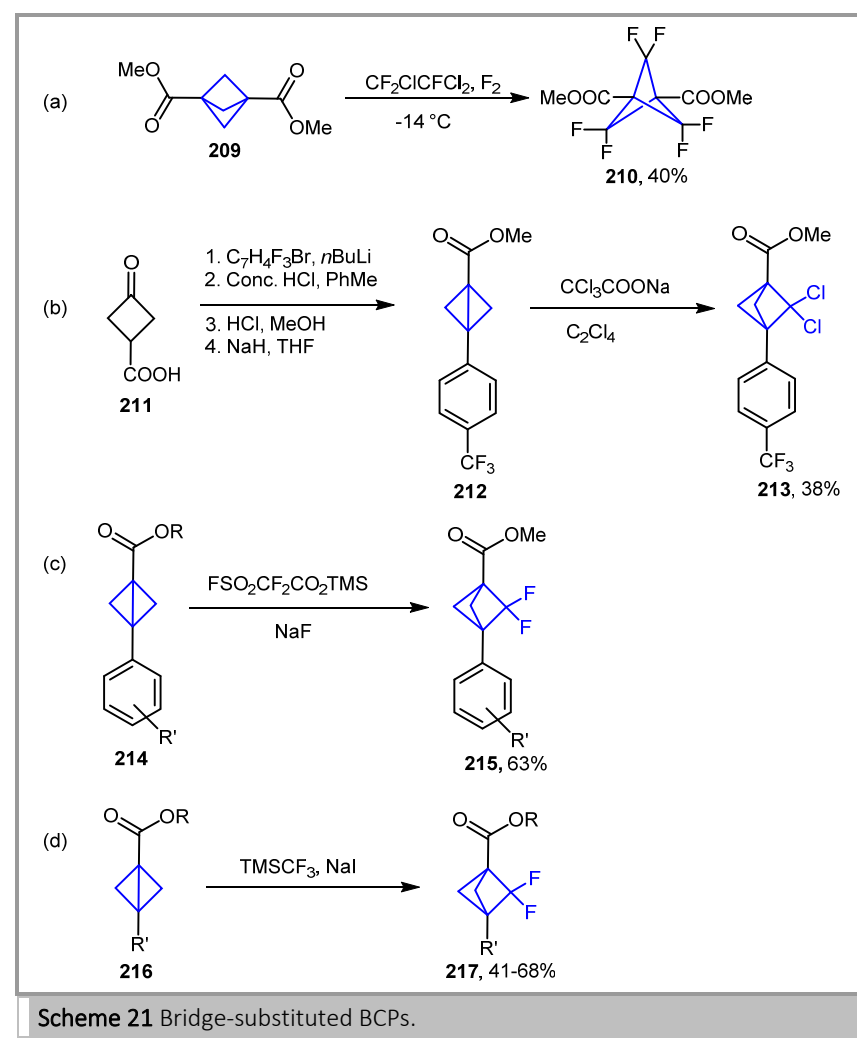

\section{Adamantane}

\subsection{Adamantane Synthesis}

Adamantane (tricyclo[3.3.1.11,7]decane) (3) a rigid yet strainfree, highly symmetric hydrocarbon has garnered significant interest in the past twenty years. ${ }^{96}$ The structure of adamantane consists of four cyclohexane rings fused into a chair conformation. The adamantane molecule exhibits a tetrahedral geometry and its 10 carbon and 16 hydrogen atoms are distinguished by two equivalent positions, i.e. the bridge and the bridgehead. In 1933, this structural analog of diamond was isolated from petroleum naphtha by fractional distillation and subsequent freezing. ${ }^{97}$ However, the yields of isolation were quite low in most cases. The pivotal synthesis of adamantane was documented by Schleyer in 1957. This protocol started from the hydrogenation of $\mathbf{2 1 8}$ followed by $\mathrm{AlCl}_{3}$-catalyzed isomerization of endo-trimethylenenorbornane (219) to access the adamantane (3) structure (Scheme 22). ${ }^{98}$ Interestingly, adamantane was one of the few compounds whose structure was effectively established from melting point analysis.

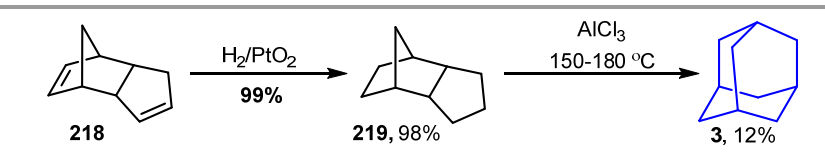

Scheme 22 Synthesis of adamantane.

\subsection{Adamantane Functionalization}

Due to the inherent reactivity of the adamantine core, $\mathrm{CH}$ functionalization is comparatively facile. However, despite considerable methods development, most of the reported procedures involve the functionalization of mono-substituted adamantanes. It has been reported that the tertiary positions 
(four $\mathrm{CH}$ positions) are highly selective towards nitration and bromination; in contrast, chlorination selectivity depends upon the solvent system. ${ }^{99}$ Additionally, it was observed that further selective substitution can be effected by bromination. ${ }^{99}$ c 1 -Halo substituted adamatanes were subjected to standard functional group transformations to yield a variety of mono substituted adamantanes. ${ }^{99 \mathrm{~d}}$

A few years later after Schleyer's seminal synthesis, the discovery of aminoadamantane and adamantane's antiviral properties facilitated their entry into medicinal chemistry. ${ }^{96}$ Moreover, insecticidal properties of chloroadamantane derivatives were reported in 1959.100a The remarkable structural and chemical properties of adamantane including its well-defined geometry, 3D orientation, hydrophobicity, and lipophilicity allows for transport across biological membranes. The biomedical properties of adamantine derivatives have been extensively explored, studied and reviewed. ${ }^{96}$ Particularly, Schreiner and coworkers documented an in-depth study on adamantane-derived pharmaceuticals. Notably, most of the pharmaceutically relevant adamantane derivatives are aminoadamantanes or adamantane carboxylic acids due to their facile and robust synthesis. Beyond their pharmaceutical importance, adamantane derivatives have been used in the development of catalysts. The 3D geometry, ready availability, and cost-effective functionalization make adamantane an appropriate candidate for incorporation into a catalyst. E.g., the use of organoadamantane catalysts has shown great success in oxidative addition, reductive elimination, and $\beta$-hydride elimination. ${ }^{100 \mathrm{~b}}$ It was observed that the electron-donating nature and steric bulk play an important role in the various step of the catalytic process. Williams and coworkers have reviewed the catalytic properties of adamantane.100c On other hand, triand tetrasubstituted adamantanes have been effectively employed in material science for the synthesis of supramolecular assemblies. The functionalization pattern, as well as the number of substitutions on the adamantane skeleton, is crucial for targeted applications. Hence, adamantane functionalization is divided according to number of substitutions at the adamantane core.

\subsubsection{1-Substituted Adamantanes}

Methods development for the controlled/selective introduction of halogen bonds at tertiary carbons is a vibrant area of research.101 1-Bromoadamanatane (220) was obtained by the direct bromination of adamantane (3). 1,3-Dibromoadamantane (221) was obtained by bromination in the presence of $\mathrm{BBr}_{3}$ whereas, tribromoadamantane (222) and tetrabromoadamantane (223) were synthesized via bromination in the presence of aluminum bromide (Scheme 23).102 Bromination proceeds via the generation of the carbonium ion, which facilitates bridgehead selectivity.

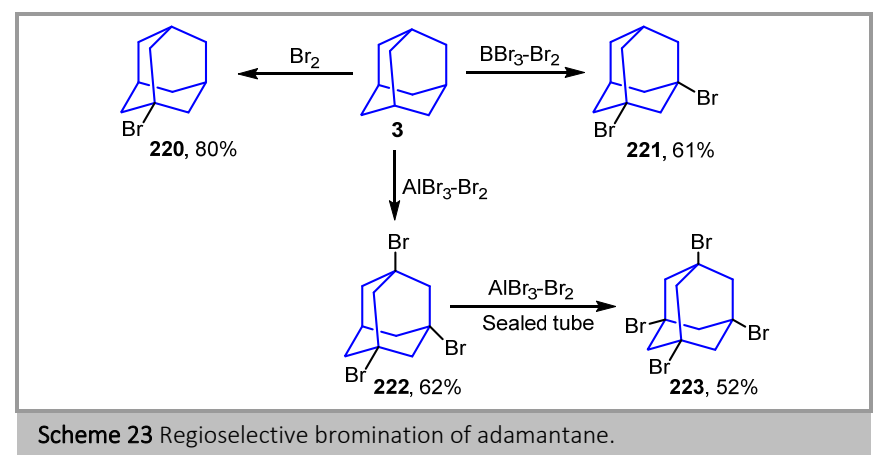

Stetter et al. utilized the Ritter reaction to synthesize $N$-(1adamantyl)acetamide (224) from 1-bromoadamantane (220) (Scheme 24a).103 A range of monosubstituted adamantane derivatives (acid, amine, amide, halogen, etc.) were synthesized using standard synthetic procedures and most of these derivatives are commercially available at present.103 The replacement of bridgehead halogen substituents (especially bromo and iodo) with other functional groups by generating carbocationic, carbanionic, and radical intermediates has been reported in the literature. ${ }^{104}$

(a)

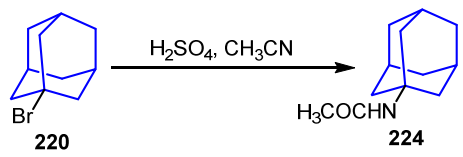

(b)<smiles>OC1(Br)C2CC3CC1CC(C=Cc1ccccc1)(C3)C2</smiles>

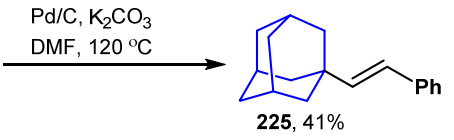

(c)
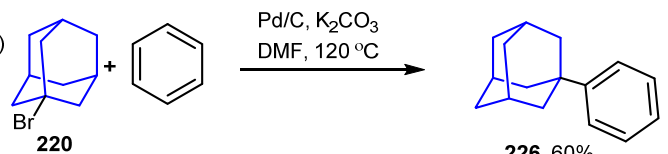<smiles>BrC12CC3CC(C1)CC(Br)(C3)C2</smiles>
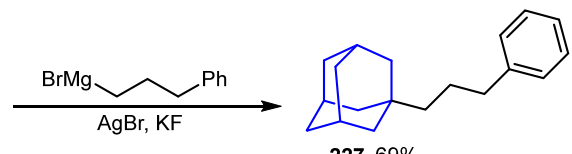<smiles>ClC12CC3CC(CC(C3)C1)C2</smiles><smiles>CC(C)c1cccc(C(C)C)c1N1C=CN(c2c(C(C)(C)C)cccc2C(C)(C)C)C1</smiles><smiles>BrC12CC3CC(C1)CC(Br)(C3)C2</smiles>

228

$229,84 \%$

(f)

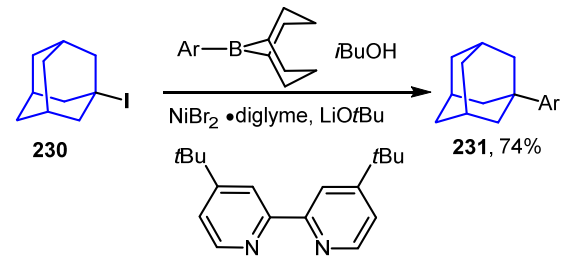

Scheme 24 Transition metal catalyzed coupling reactions with 1 haloadmantane.

In contrast to 1-halocubanes, 1-haloadamantanes (220, 228 and 230) can be used as electrophiles in metal-catalyzed coupling reactions. ${ }^{105}$ For example, the $\mathrm{Pd} / \mathrm{C}$-catalyzed coupling of 1bromoadamantane (220) with styrene was quite successful and 
yielded a variety of (E)-configurated alkenes (225) (Scheme $24 \mathrm{~b}$ ). These reactions proceed via a cationic mechanism; indeed, 1-phenyladamantane (226) was synthesized when 1bromoadamante (220) was reacted with benzene under the same reaction conditions (Scheme 24c). Transition metal catalyzed coupling reactions of Grignard reagents with tertiary alkyl halide is a useful method for the generation of C-C bonds at unreactive tertiary carbon positions. The silver catalyzed $(\mathrm{AgBr} / \mathrm{KF})$ coupling reaction of the 1-bromoadamantane (220) with three eq. of (3-phenylpropyl)magnesium bromide yielded 1-(3'-phenylpropyl)adamantane (227) in a 69\% yield (Scheme 24d). ${ }^{106}$

In another example, the reaction of 1-chloroadamantane (228) with arylmagnesium bromide in the presence of catalytic $\mathrm{FeCl}_{3}$ and N-heterocyclic carbene gave 1-aryladamantane (229) (Scheme 24e). ${ }^{107}$ Reactions of organoboron reagents with alkyl halides are extensively exploited in Suzuki or Suzuki type crosscoupling reactions. Zultanski and Fu applied the coupling of 1iodoadamantane (230) with substituted 9borabicyclo[3.3.1] nonane (9-BBN) reagents in the presence of a Ni-catalyst $\left(\mathrm{NiBr}_{2}\right.$-diglyme/4,4'-di-tert-butyl-2,2'-bipyridine) to synthesize 1-aryladamantane (231) (Scheme 24f). However, the authors showed that 1-bromoadamantane was not a suitable coupling partner under these coupling conditions. ${ }^{108 a} \mathrm{~A}$ reaction of 1-iodoadamantane or 1-bromoadamantane with silver(I) phenylacetilyde in N-methylmorpholine (NMM) afforded the synthesis of 1-(phenylethynyl)adamantine in $68 \%$ yield.108b,108c These reactions have a broad substrate scope and allow the synthesis of the respective adamantane derivatives in yields ranging from 25 to $68 \%$.

The above-mentioned examples show the utility of 1haloadamantanes as (pro)electrophiles in metal catalyzed coupling reactions., the conversion of 1-haloadamantanes into organometallic reagents is rather appealing due to their reactivity towards a broad range of electrophiles. Despite considerable progress in diamondoid chemistry, the synthesis of organometallic reagents of adamantane is still a challenge. The reaction of 1-bromoadamantane with Rieke's magnesium yielded adamantane and 1,1'-biadamantane (232) but no trace of the adamantylmagnesium compound was observed (Scheme 25a). Hence, Dubois and coworkers developed a "static method" to synthesize adamant-1-yl magnesium bromide (233), which undergoes reactions with standard electrophiles including benzaldehyde to yield the corresponding alcohol (234) (Scheme 25b). ${ }^{109}$

The treatment of 1-bromoadmantane (220) with Rieke's zinc readily yielded adamantylzinc bromide (235); furthermore, introduction of the adamantylzinc reagent to a $\mathrm{CuCN} / \mathrm{LiBr}-$ catalyzed cross-coupling reaction with benzoyl chloride gave the adamantyl phenyl ketone (236) (Scheme 25c). ${ }^{110}$ In other studies, it was observed that 1-adamantylmagnesium chloride (237) tolerated a number of functional groups during $\mathrm{Ni}$ catalyzed coupling reactions. E.g., the $\mathrm{Ni}(\mathrm{acac})_{2}$ catalyzed reaction of a Grignard reagent with haloarenes in the presence of NHC (1-(2'-carboxyethyl)-3-phenyl-1H-imidazol-3-ium chloride) and LiOtBu produced the aryladamantane derivatives (238) in 58-85\% yields (Scheme 25d). ${ }^{111}$ Most recently, Knochel and coworkers developed an elegant method for the synthesis of organometallic adamantane complexes.112a The LiCl mediated reaction of 1-bromoadamantane with $\mathrm{Mg}$ in the presence of $\mathrm{ZnCl}_{2}$ led to the formation of the corresponding $\mathrm{Zn}$ complex 239 in a $85 \%$ yield. Zinc complex 239 was subjected to a Buchwaldmodified Negishi cross-coupling reaction ${ }^{112 b}$ to achieve the synthesis of 1 -substituted adamantane (240) (Scheme 25e).

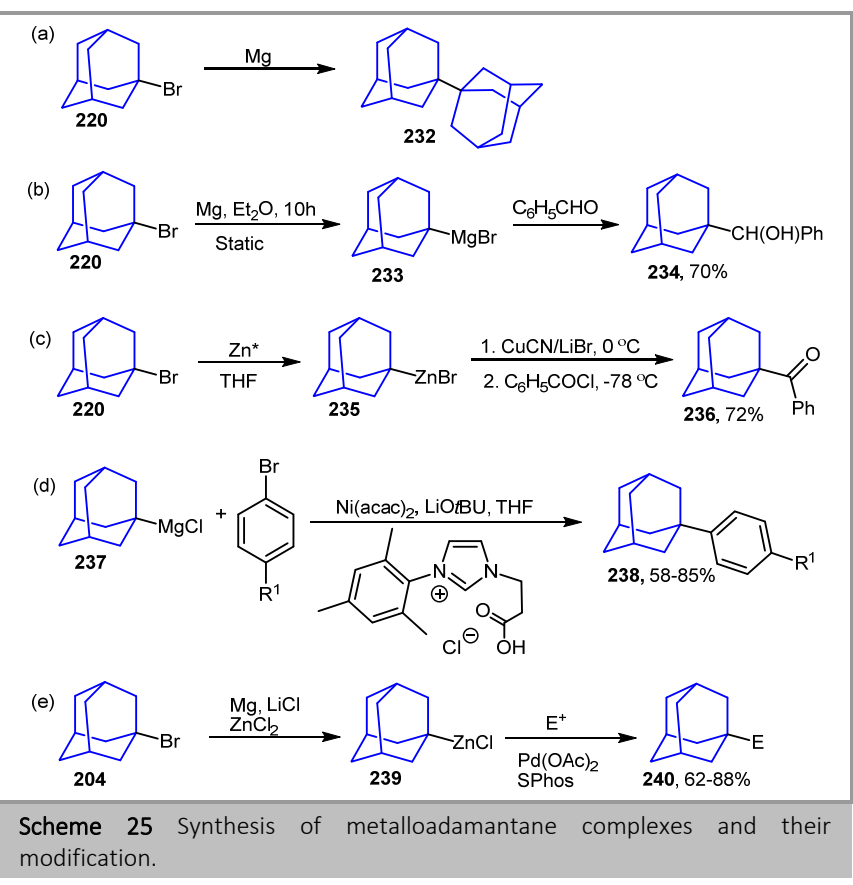

The adamantane radical exhibits nucleophilic character; hence, reaction with an electrophilic unsaturated bond is quite favorable. The reaction of 1-adamantyl bromide and iodide with alkenes and alkynes containing electron-withdrawing groups in the presence of tributyltin hydride and azobisisobutyronitrile (AIBN) afforded the alkane or alkene substituted adamantane. MacMillan and coworkers reported the direct arylation of adamantan-2-one (241). A combination of light and $\left[\mathrm{W}_{10} \mathrm{O}_{32}\right]^{4-} / \mathrm{Ni}(\mathrm{dtbbpy}) \mathrm{Br}_{2}$ facilitate the reaction of 5 -bromo-2(trifluoromethyl)pyridine with adamantan-2-one (241) to give 5-(6'-(trifluoromethyl)pyridin-3'-yl)adamantan-2-one (242) in a $48 \%$ yield (Scheme $26 \mathrm{a}) .{ }^{113}$

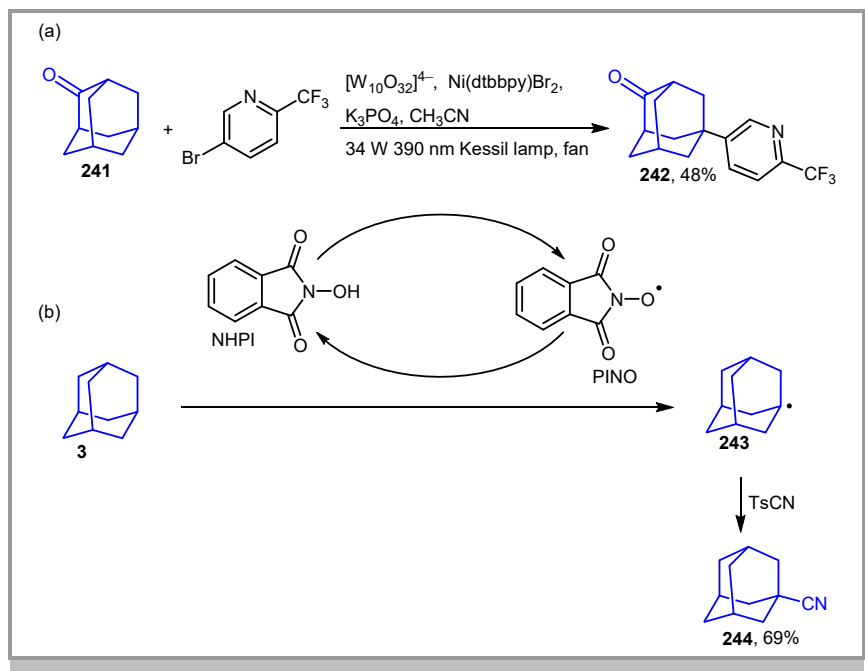

Scheme 26 Photoredox and chemical generation of adamantly radical. 
Schreiner and coworkers reported the phthalimido-N-oxyl (PINO) catalyzed selective $\mathrm{C}_{\mathrm{sp} 3}-\mathrm{H}$ cyanation of adamantane. This procedure involved the generation of PINO from NHPI, followed by hydrogen atom transfer (HAT) to PINO. The HAT from adamantane to PINO generates the adamantly radical (243), which was trapped by $p$-toluenesulfonyl cyanide (TsCN), thereby affording the desired 1-cyanoadamantane (244) (Scheme 26b). ${ }^{114}$ Recently, Martin and coworkers reported the reaction of 1-substituted adamantane with a phenyl vinyl sulfone using an iridium photocatalyst and quinuclidin-3-yl benzenesulfonate as the $\mathrm{H}$ atom transfer catalyst. ${ }^{115}$

\subsubsection{1,3-Disubstituted Adamantanes}

In contrast to disubstituted-BCP, cubane and bicyclo[2.2.2] octane (BCO) the two substituents at the adamantane bridgehead positions adopt a tetrahedral orientation with an angle of $109.5^{\circ}$. Due to this spatial arrangement of functional groups, disubstituted adamantanes can act as a building block for the synthesis of large macrocyclic ring or spheres of suitable size. The adamantane based macrocycles have potential applications in molecular recognition and host-guest chemistry. 1,3-bisphenol derivatives of adamantanes (245 and 246) used as building blocks for synthesis of adamantane-based oxacyclophanes $(\mathbf{2 4 7 , 2 4 8 )}$. The macrocycles 247 and 248 were synthesized via reaction of bisphenol adamantane derivatives $(\mathbf{2 4 5}, \mathbf{2 4 6})$ with 2,6dichloropyrazine in the presence of cesium carbonate (Scheme 27a). ${ }^{116 a}$ The presence of both polar pyrazine and non-polar adamantane moieties act as an driving force to form aggregates in organic media. An acetone solution of the adamantane-based oxacyclophanes $(\mathbf{2 4 7}, \mathbf{2 4 8})$ was treated with water to induce the formation of spherical, fibrous, and network aggregates which ultimately led to single crystals. This conversion indicates a phase transition from solution to solids via a hollow sphere. ${ }^{116 a}$ Salen-adamantane based macrocycles 251 also exhibit solvent-dependent self-assembly formation. A Sonogashira reaction between 2-hydroxy-4-iodobenzaldehyde and 1,3-diethynyladamantane (249) gave 4,4'-(adamantane-1,3diylbis(ethyne-2,1-diyl))bis(2-hydroxy benzaldehyde) (250). The final key step involved a Schiff-base coupling reaction between bis-adamantylaldehyde (250) and diethylamine and yielded the macrocycle 251 (Scheme 27b).116b The addition of methanol to a THF solution of the macrocycle $\mathbf{2 5 1}$ led to the formation of crystals of the hollow spherical and fused aggregates, while the addition of chloroform did not lead to any self-assembly.

Along the same line, the incorporation of adamantane into a phenol containing macrocycle was achieved in two steps. The first step involved an Ullmann coupling reaction between 4,4' bisiododphenyl and 4,4'-(adamantane-1,3-diyl)bis(2,6dimethoxyphenol) (246) and gave the 1,3-bis(4'-(4"iodophenoxy)-3,5-dimethoxyphenyl) adamantane (235). ${ }^{116 c}$ In the final step, 1,3-bis(4'-(4"-iodophenoxy)-3,5dimethoxyphenyl)adamantane (252) underwent a $\mathrm{Ni}(\mathrm{cod})_{2}$ catalyzed self-coupling reaction to form the macrocycle (253) (Scheme 27c). Like 247 and 248, the self-aggregation properties of macrocycle $\mathbf{2 5 1}$ were also dependent upon the organic media. 1,3-Bisethynyladamantane (249) was utilized for the macrocycle construction and coupling with 4bromoisophthalate resulted in bis-isophthalateadamantane.
Subsequent bromination and cyclization afforded the pyrene substituted adamantane.117 Interestingly, this hybrid structure exhibits a large dipole moment due to orthogonal arrangement of 1,3-disubstituted adamantane and pyrene moiety.

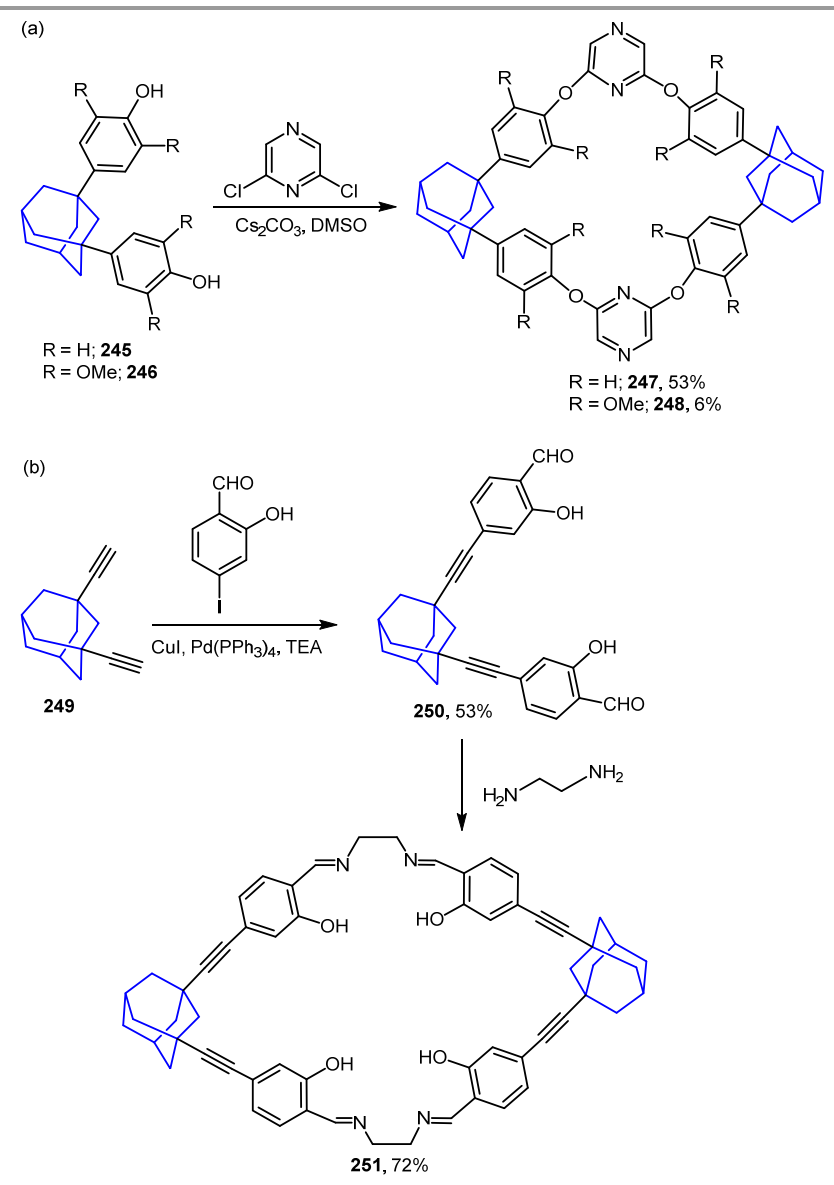

(c)

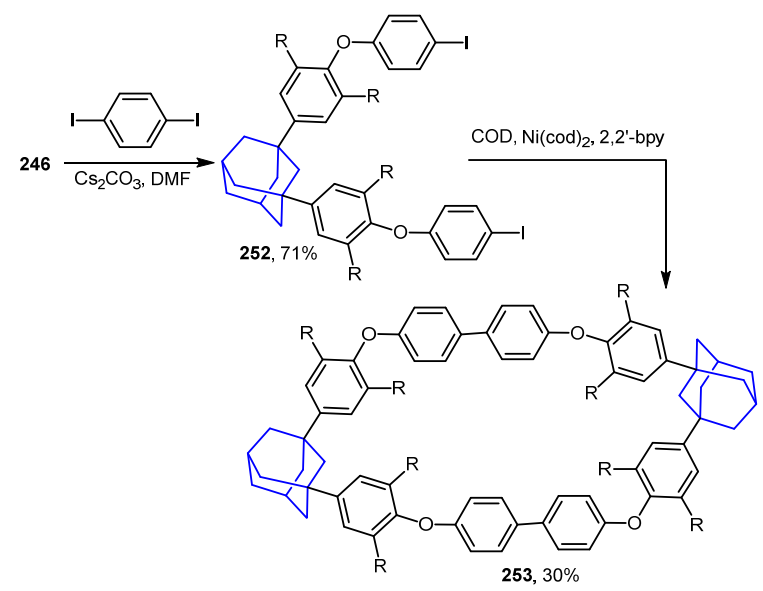

Scheme 27 Adamantane-based cyclophanes.

Incorporation of a 1,3-bisethynyladamantane (249) unit between two pentacene units led to the synthesis of a nonconjugated covalently linked pentacene dimer (256). The addition of lithium triisobutylsilylacetylene to 6,13pentacenequinone (253) provided access to 13-methoxy-13'(isobutylsilylacetylene)-pentacene-6-one (258). Further reaction of bis-lithium adamantane-1,3-diylbis(ethyn-1-ide) (255) with monoketone pentacene followed by tin(II) mediated 
reductive aromatization yielded the pentacene dimer (256) Linkage of two pentacene units through non-conjugated adamantane unit inhibits the undesired overlapping of the frontier molecular orbital of each pentacene. Hence, singlet and triplet states of each individual chromophore survive in the dimer 256 which promoted sufficient electronic coupling between the two pentacene units, rendering singlet fission. (Scheme 28).118

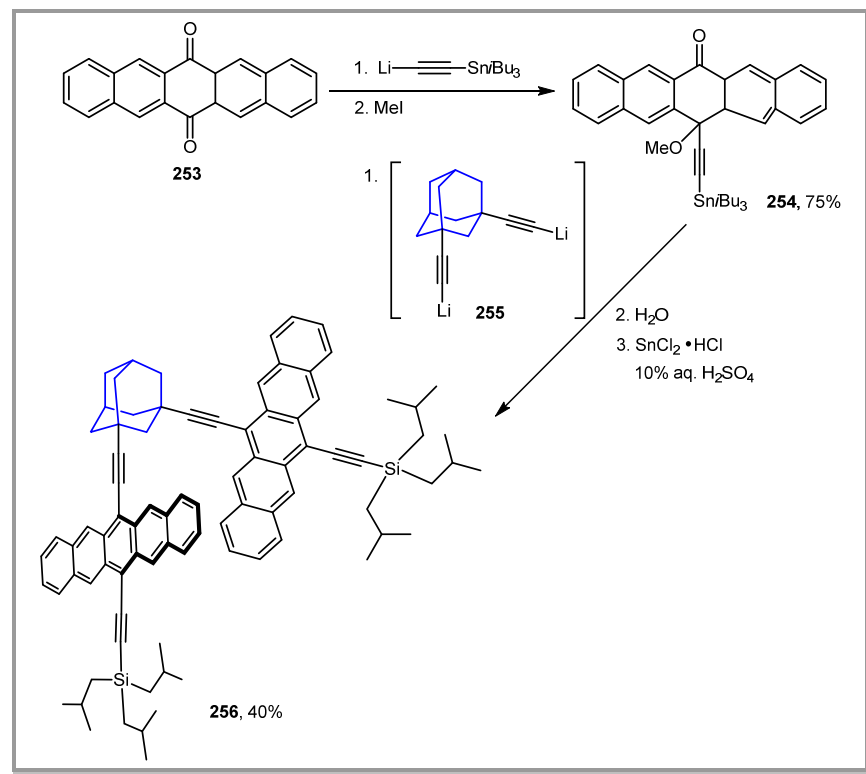

Scheme 28 Synthesis of an adamantane linked pentacene dimer.

\subsubsection{1,3,5-Trisubstituted Adamantanes}

Introduction of three substituents at 1,3,5-positions of adamantane adopts a tripodal geometry. Furthermore, three identical functionalities at the adamantane bridgehead introduces a $\mathrm{C}_{3}$ symmetry. Trisubstituted adamantanes exhibit tripodal geometry which is used for drug delivery systems and surface recognition studies.119 Friedel-Crafts arylation of 1bromoadamantane (220) led to the formation of 1,3,5triphenyladamantane (257). ${ }^{120 a}$ Adamantane-1,3,5-carboxylic acid (258) was synthesized via the $\mathrm{RuCl}_{3} / \mathrm{H}_{5} \mathrm{IO}_{6}$-catalyzed oxidation of a 1,3,5-triphenyladamantane derivative (257). Furthermore, the triacid was converted into the triester (259) and triol (260) using standard chemical transformations (Scheme 29a). A reaction of 1,3,5-tribromoadamanatne (223) with vinyl bromide gave the 1,3,5-triethynyladamantane (261) which is a precursor for large complex architectures 266 and 269.120 1,3,5-triethynyladamantane (261) was reacted with $\mathrm{MeLi} / n \mathrm{BuLi}$ followed by addition of various electrophiles including carbon dioxide $\left(\mathrm{CO}_{2}\right)$, paraformaldehyde $\left(\left(\mathrm{CH}_{2} \mathrm{O}\right)_{\mathrm{n}}\right)$, 4-toluenesulfonyl chloride ( $\mathrm{TsCl}$ ) to access a variety of $\mathrm{C}_{3}$ symmetric building blocks $(\mathbf{2 6 2}, \mathbf{2 6 3}, \mathbf{2 6 4})$ (Scheme 29b).

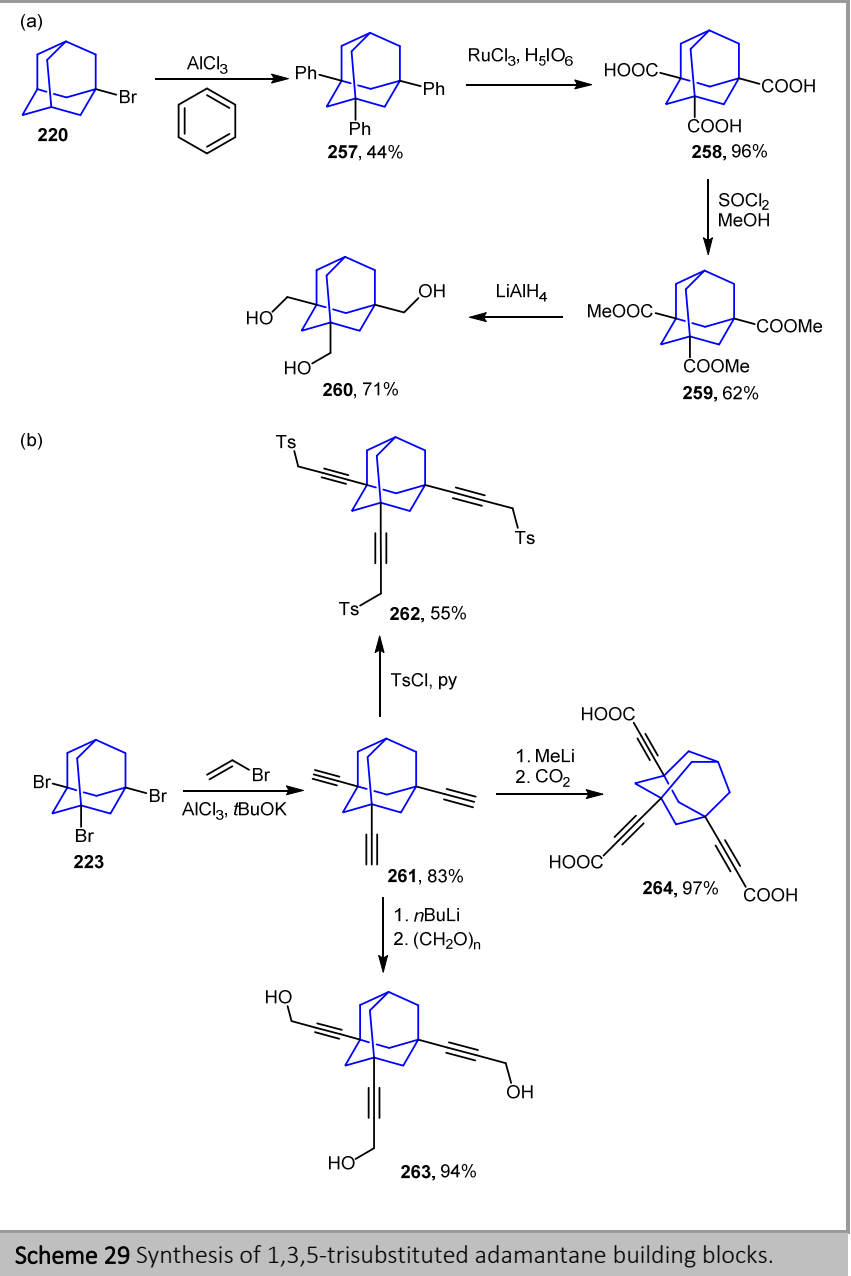

Scheme 29 Synthesis of 1,3,5-trisubstituted adamantane building blocks. 
A standard Sonogashira coupling reaction between 4bromopyridine hydrochloride and 1,3,5-triethynyladamantane (261) yielded the tritopic pyridine linker (265). A trigonal metal-directed supramolecular self-assembly system 266 can be constructed from two equiv. of tritopic $109^{\circ}$ angular adamantane and three equiv. of ditopic $90^{\circ}$ angular metals (for example cis-Pt($\left(\mathrm{PMe}_{3}\right)_{2}(\mathrm{OTf})_{2}, \quad$ cis-Pt(dppe)(OTf $)_{2}, \quad$ or $\quad$ cisPt(dppp)(OTf)2.) (Scheme 30a).121a Along similar lines, 1,3,5triethynyladamantane was coupled with 2,2'-bispyridine to yield a tritopic bispyridyl linker. ${ }^{121 b}$
Additionally, the introduction of appropriate substituents leads to the formation of non-covalent networks through $\mathrm{CH} / \mathrm{O}$ interactions between the cage molecules. As discussed earlier, 1,3,5-triethynyladamantane (261) has proven to be a versatile scaffold. The Sonogashira coupling of 3-iodo-5[(trimethylsilanyl)ethynyl] benzoic acid methyl ester with 1,3,5triethynyladamantane afforded a monomer unit (267). Deprotection followed by Glaser/Hay coupling of the monomer provided access to the macrocyclic cage (269) (Scheme 30b). ${ }^{122}$

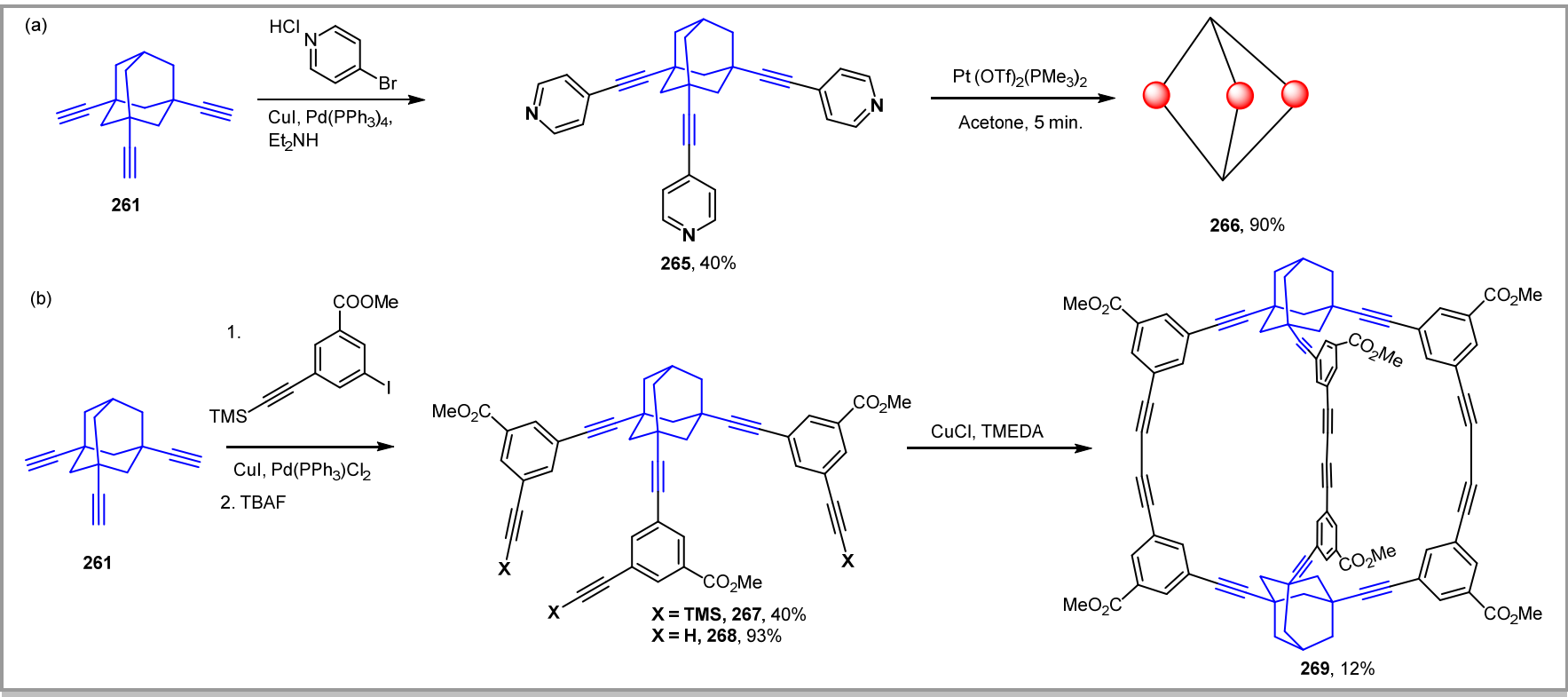

Scheme 30 Synthesis of tritopic adamantane linkers and macrocycles.

\subsubsection{1,3,5,7-Tetrasubstituted Adamantanes}

An interesting feature of tetravalent adamantane is the arrangement of substituents in tetrahedral arrangement; hence, adamantane core is often considered as a large surrogate of $\mathrm{sp}^{3}$ carbon. Tetrasubstituted-adamantane derivatives have been used in several applications including catalysis, surface functionalization, biocompatible surfaces, and material science. ${ }^{123}$ Here, 1,3,5,7-tetrabromoadamantane (207) is the key compound which can be further functionalized to afford the synthesis of other molecular tectons such as 1,3,5,7tetraphenyladamantane (253), 1,3,5,7-tetracyanoadamantane (254), 1,3,5,7-tetraaminoadamantane (255), and adamantane1,3,5,7-tetracarboxylic acids (256) (Scheme 31a). 


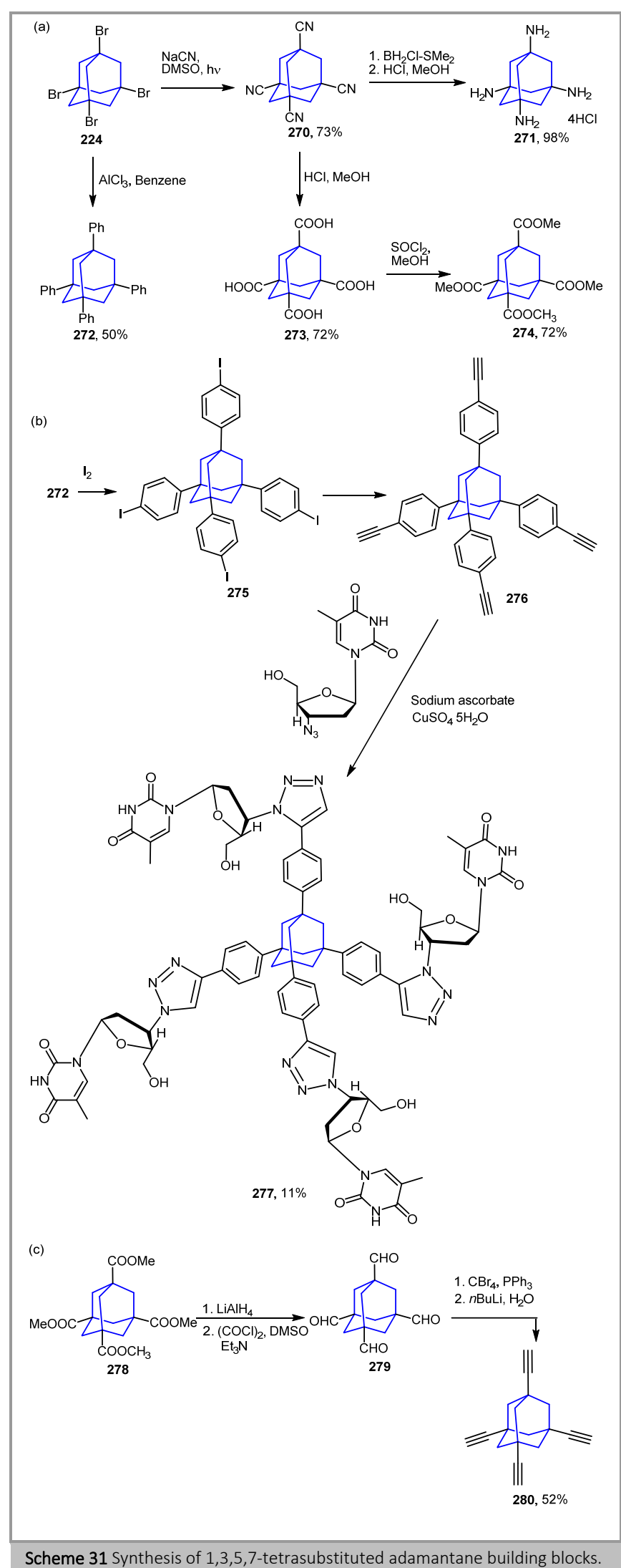

As discussed in previous sections, direct nucleophilic substitution or radical reactions allows for selective derivatization at the bridgehead positions. The adamantane radical is nucleophilic is nature and undergoes facile $\mathrm{S}_{\mathrm{N}} \mathrm{R}^{1}$ reactions. 1,3,5,7-Tetracyanoadamantane (253) was synthesized via a photochemical reaction of sodium cyanide
(NaCN) with 1,3,5,7-tetrabromoadamantane (224) (Scheme 31a) ${ }^{124}$ and it can be easily converted into carboxylic acid (256) and amino derivatives (256). Similarly, 1,3,5,7tetraaminoadamantane, 1,3,5,7-acetamidoadamantane, and 1,3,5,7-tetracarbomethoxy adamantine can be synthesized on multi-gram scales using standard procedures. ${ }^{125}$

Appending phenyl groups at the bridgehead positions is the most common way to maintain rigidity of adamantane core, while still providing further functionalization pathways. Moreover, phenyl groups reduce steric and electronic demands when trying to perform direct functionalization at the saturated core. The para-position of these phenyl rings can be easily iodinated or brominated in quantitative yields. ${ }^{126 a}$ 1,3,5,7tetrakis(4'-iodophenyl)adamantane (258) and 1,3,5,7tetrakis(4'-ethnylphenyl)adamantane (259) have been used as key organic tectons to form self-assemblies. ${ }^{126 b}$ Compounds 275 and 276 have participated in a number of standard reactions including Pd-catalyzed Suzuki reactions, Sonogashira couplings, click reactions, and multicomponent cascade reactions. ${ }^{127}$ The four-fold click reaction of tetrakis(4-ethynylphenyl)adamantane (276) and 3'-azido-2',3'-dideoxynucleosides (thymidine and cytidine) gave the corresponding triazoles (277) (Scheme 31b). These tetranucleosides were further subjected to solution-phase DNA-synthesis, which demonstrated the utility of the adamantane core in DNA-based nanostructuring. ${ }^{127 c}$

Atomic force microscopy (AFM) is a relatively new molecular imaging technique utilized for broad applicability. ${ }^{128}$ The sharpness of the AFM tip is a major factor in controlling the resolution of this technique. Keana and coworkers reported that a tetrahedrally shaped adamantane molecule, derivatized with $\mathrm{S}$-atoms at the termini of the tripodal could be employed for AFM. Given the high affinity of the S-atom for gold, the Sfunctionalized termini eventually anchored the molecular tip to a thin film of gold placed onto a conventional AFM tip. ${ }^{129}$ Sonogashira coupling of tetraethynyladamantane with a 4(acetylsulfanylmethy)phenyl or 3,5-bis(acetylsulfanylmethyl) phenyl unit delivered the S-functionalized adamantane arms, which constructed the monolayers on the gold surface. Moreover, an adamantane unit containing fluorescent units at the bridgehead positions amplified the fluorescence. ${ }^{130}$

1,3,5,7-tetraethynyladamantane (282) is another key building block in the synthesis of non-conjugated linked multichromophoric systems. ${ }^{131}$ 1,3,5,7-Tetraethynyladamantane (280) was synthesized from adamantane-1,3,5,7tetracarbaldehyde (279) using the Corey-Fuchs reaction. The reaction of lithium bis(trimethylsilyl)amide with 1,3,5,7tetraethynyladamantane gave 1,3,5,7tetralithiumethynyladamantane (263). Following, this key compound was reacted with 13-metoxy-13'-(isobutylsilylacetylene)-pentacene-6-one (238) and the crude reaction mixture was subjected to reductive elimination to afford the target adamantane linked pentacene tetramer $\mathbf{2 8 2}$ in $18 \%$ yield (Scheme 32). Spectroscopic investigations of this tetramer showed that efficient singlet fission in the solid state occurred. 


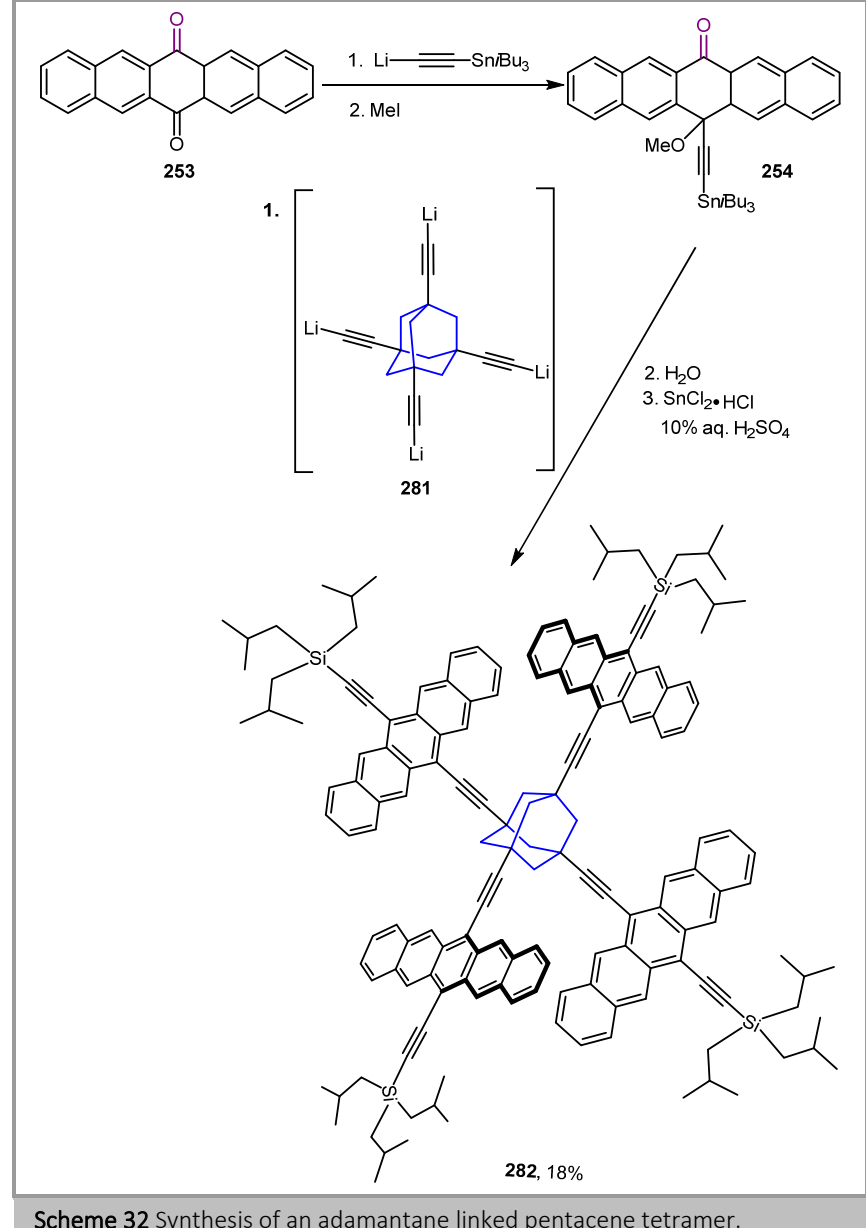

These examples described the synthesis and utility of symmetric adamantane derivatives. The four tertiary carbons at adamantane skeleton provide many possibilities to target the synthesis of multi-functional adamantane derivatives. Maison and coworkers reported the synthesis of unsymmetrically substituted adamantane derivatives which have three identical substituents arranged in a tripodal manner and fourth orthogonal substituent arranged in opposite direction. The three identical arms at adamantane skeleton can act as anchors to surface whereas fourth functionality can conjugate to drugs, dyes or polymers units (Figure 3). Due to the incorporation of three similar functionalities and one different substituent, these derivatives named as $(3+1)$ adamantane building blocks.

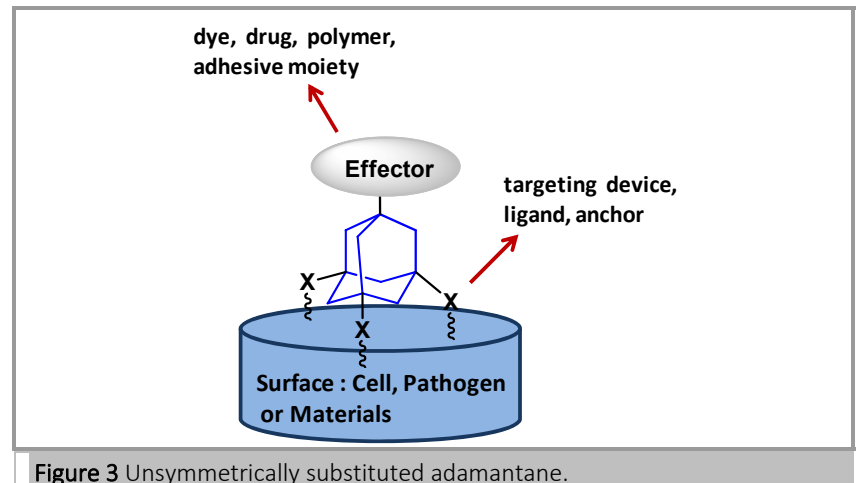

Figure 3 Unsymmetrically substituted adamantane.

These $(3+1)$ adamantane derivatives $(\mathbf{2 8 7 , 2 8 9})$ have been applied in tumor imaging, targeted tumor therapy, and material chemistry. ${ }^{132}$ There are three routes to synthesize such $(3+1)$ building blocks: (a) desymmetrization of the tetrasubstituted adamantanes, (b) substitution at the fourth position of tripodal adamantane, and (c) functionalization of monosubstituted derivatives. Even though several low-cost monosubstituted adamantane derivatives are available, route (c) has not yet been extensively explored. Maison and coworkers have explored routes (a) and (b) to synthesize a library of $(3+1)$ derivatives with orthogonal functionalities including cyano, amino, carboxylic acid, hydroxyl, and alkyne units with spacer chains of different lengths. ${ }^{133 a}$ The selective acylation of 1,3,5,7tetrakis(aminomethyl)adamantane is an example of the route (a). ${ }^{133 b}$. The mono bromination of 1,3,5-triphenyladamantane (249) yielded the 1-bromo-3,5,7-triphenyladamantane. The bromo functionality was interconverted to an acetamide group using Ritter reaction conditions to afford the $\mathrm{N}-(3,5,7-$ triphenyladamantan-1-yl)acetamide (283) (Scheme 33). Furthermore, ruthenium(III) chloride/orthoperiodic acid $\left(\mathrm{RuCl}_{3} / \mathrm{H}_{5} \mathrm{IO}_{6}\right)$ catalyzed oxidative degradation of phenyl group and acetamide hydrolysis provides access to 7aminoadamantane-1,3,5-tricarboxylic acid (284). In the next step, synthesis of $\mathbf{2 8 6}$ was achieved by amide coupling with methyl 6-aminohexanoate.

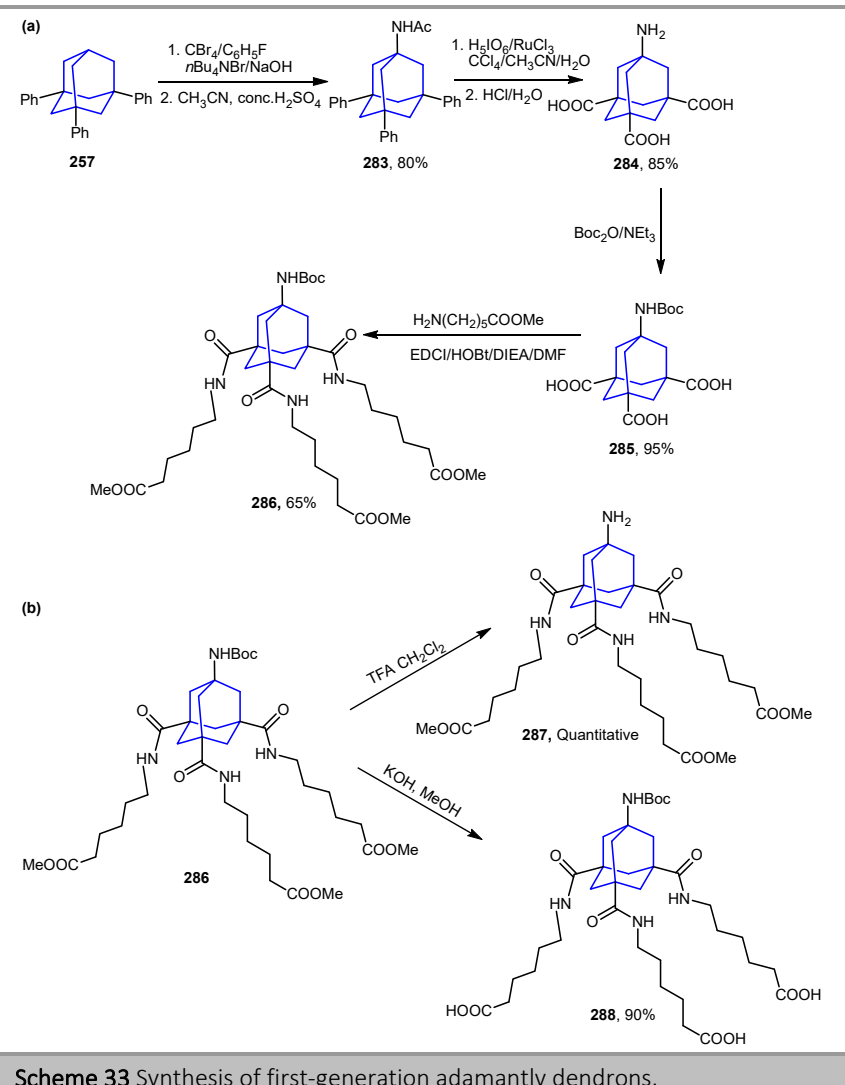

Compound 286 is an example of first-generation adamantanebased dendron (HYDRAmers) that was smoothly converted into water-soluble derivatives $\mathbf{2 8 7}$ and $\mathbf{2 8 8}$. The water-soluble moieties $(\mathbf{2 8 7}, \mathbf{2 8 8})$ were further employed for the synthesis of second-generation dendrons (289) with multiple adamantane units (Scheme 34). 


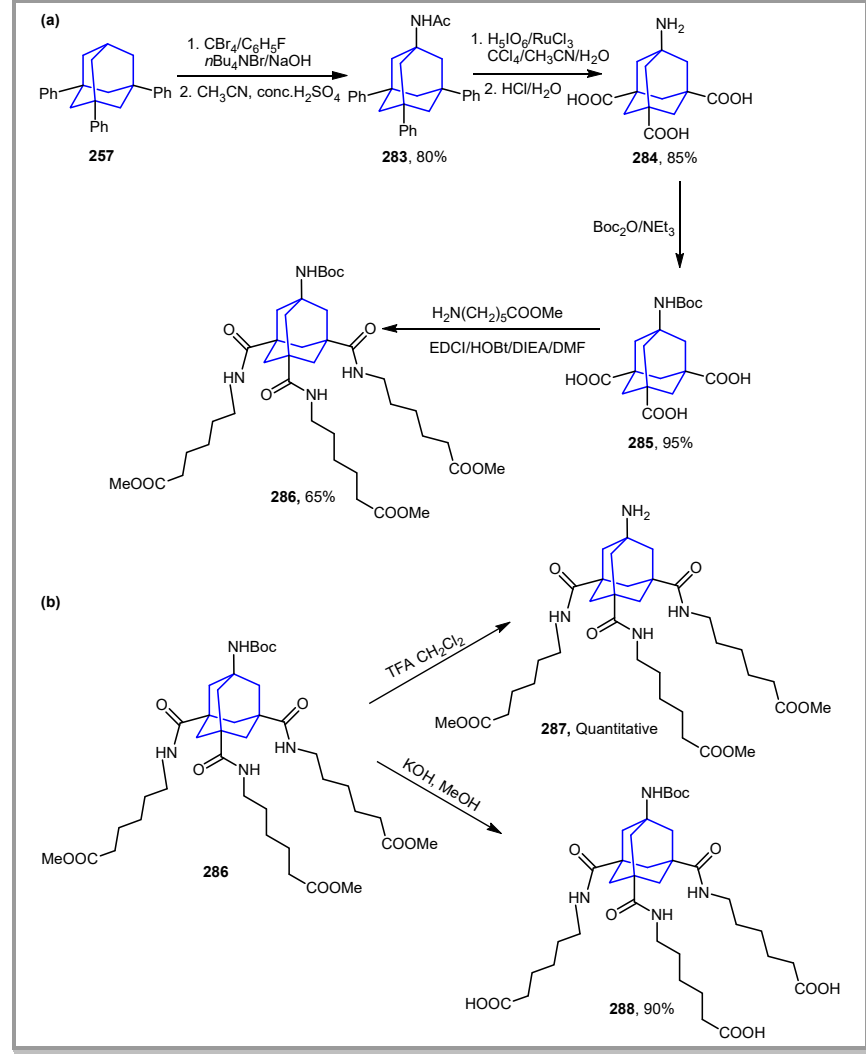

Scheme 34 Synthesis of second-generation adamantly dendrons.

\section{Conclusions and Outlook}

In the last five and a half decades we have witnessed the development of methodologies for the synthesis and functionalization of rigid scaffolds. What started as a laboratory curiosity is now a rapidly growing field of molecular tectons and synthons and we are progressively moving from purely academic to multiple application-based studies. Indeed, the development of robust protocols and the commercial availability of diester derivatives of cubane (1) and BCP (2) has allowed for a number of caged hydrocarbons to be exploited for further chemical transformations including the Barton reaction, Corey-Fuchs reaction, and radical cross-coupling reactions (RCC or SET). A question of fundamental significance is the difference between the nature of bridgehead carbons of compounds 1,2 , and 3. The answer can be explored by analyzing \%s-character and bond angles. Bridgehead carbons of cubane (1) have 31\% scharacter with $90^{\circ}$ bond angle, bridgehead carbons of BCP (2) have $34 \%$ s-character with $87-88^{\circ}$ bond angle, whereas bridgehead carbons of adamantane have $26 \%$ s-character with $109-110^{\circ}$ bond angle.

The $\mathrm{CH}$ activation at the cubane skeleton is quite challenging due to the highly strained $\mathrm{sp}^{3}$-carbons. The cubane skeleton is inert towards standard organometallic reagents. Ultimately, radical cross-coupling emerges as a novel approach to yield the aryl-substituted cubanes. Additionally RCC coupling proved as a useful synthetic tool for the synthesis of porphyrin-cubane arrays.

In the past few years, BCP chemistry has undergone a renaissance. This renaissance has introduced the debut of many new types of BCP-derivatives, including amines, amino acids, sulfonamides, xanthates, and porphyrin substituted BCPs.
However, most of the synthetic protocol commenced from [1.1.1]propellane, which limits the substrate scope. Additionally, direct $\mathrm{CH}$ functionalization on BCP (2) was hardly achieved potentially due to strained and inverted tertiary carbon. Hence, synthetic methods are not yet discovered enough to limit BCP science solely to the organic chemists' imagination. We are optimistic that the new derivatives of BCP (2) will continue to be discovered.

The $\mathrm{sp}^{3}$-carbons in adamantane (3) are close to those of other saturated hydrocarbons. Hence, the adamantane core is more reactive towards standard reactions such as bromination, nitration, and Friedel-Crafts. Mono-substituted adamantanes are well explored and utilized in the pharmaceutical industry. The synthesis of symmetrically multi-substituted adamantanes is achieved while the synthesis of unsymmetrically substituted adamantanes is yet to explore.

Despite great developments in organic chemistry, the direct and facile $\mathrm{CH}$ activation of strained scaffolds, especially cubane, is still a challenging task. In many cases, the substrate scope is often limited to one derivative or entry along with other saturated alkanes, boronic esters, redox-active esters, and carboxylic acids. However, the electronic, chemical and structural properties of cyclic, rigid and non-saturated scaffolds are quite different from other flexible alkanes or rigid arenes. The high cost and limited accessibility prevent the utility of these saturated rigid linkers in industrial applications. Indeed, the first step towards these applications should be an organic chemist's endeavor to overcome the strain driven rearrangements that hamper direct scaffold functionalization.

\section{Funding Information}

This work was supported by a grant from Science Foundation Ireland (SFI IvP 13/IA/1894).

\section{References}

(1) Hopf, H. in Classics in Hydrocarbon Chemistry: Syntheses, Concepts, Perspectives, Wiley-VCH, Weinheim, 2000.

(2) Paquette, L. A. Proc. Natl. Acad. Sci. U.S.A. 1982, 79, 4495

(3) Eaton, P. E. Angew. Chem. Int. Ed. Engl. 1992, 31, 1421.

(4) (a) Paquette, L. A.; Ternansky, R. J.; Balogh, D. W.; Kentgen, G. J. Am. Chem. Soc. 1983, 105, 5446. (b) Fessner, W.-D.; Murty, B. A. R. C.; Prinzbach, H. Angew. Chem. Int. Ed. Engl. 1987, 26, 451. (c) Fessner, W.-D.; Murty, B. A. R. C.; Wörth, J.; Hunkler, D.; Fritz, H.; Prinzbach, H.; Roth, W. D.; Schleyer, P. v. R.; McEwen, A. B.; Maier, W. F. Angew. Chem. Int. Ed. Engl. 1987, 26, 452. (d) Maier, G.; Pfriem, S.; Schäfer, U.; Matusch, R. Angew. Chem. Int. Ed. Engl. 1978, 17, 520.

(5) (a) Locke, G. M.; Bernhard, S. S. R.; Senge, M. O Chem. Eur. J. 2019, 25, 4590. (b) Kanazawa, J.; Uchiyama, M. Synlett 2019, 30, 1. (c) Dilmaç, A. M.; Spuling, E.; de Meijere, A.; Bräse, S. Angew. Chem. Int. Ed. 2017, 56, 5684. (d) Biegasiewicz, K. F.; Griffiths, J. R.; Savage, G. P.; Tsanaktsidis, J.; Priefer, R. Chem. Rev. 2015, 115, 6719. (e) Levin, M. D.; Kaszynski, P.; Michl, J. Chem. Rev. 2000, 100, 169. (f) Wiberg, K. B. Chem. Rev. 1989, 89, 975.

(6) Reaxys Database, https://www.reaxys.com, downloaded 29/02/2020.

(7) (a)Davies, W. L.; Grunert, R. R.; Haff, R. F.; McGahen, J. W.; Neumayer, E. M.; Paulshock, M.; Watts, J. C.; Wood, T. R.; Hermann, E. C.; Hoffmann, C. E. Science 1964, 144, 862. (b) Tsunoda, A.; Maassab, H. F.; Cochran, K. W.; Eveland, W. C. Antimicrob. Agents Chemother. 1965, 553. (c) Fanta, D. Wien. Med. Wochenschr. 1976, 126, 315. (d) Rosenthal, K. S.; Sokol, M. S.; Ingram, R. L.; 
Subramanian, R.; Fort, R. C. Antimicrob. Agents Chemother. 1982, 22, 1031. (e) Savage, S. A.; Jones, G. S.; Kolotuchin, S.; Ramrattan, S. A.; Vu, T.; Waltermire, R. E. Org. Process Res. Dev. 2009, 13, 1169. (f) Rosenstock, J. Expert Rev. Endocrinol. Metabol. 2010, 5, 809 (g) Stockdale, T. P.; Williams, C. M. Chem. Soc. Rev. 2015, 44, 7737.

(8) (a) Lovering, F.; Bikker, J.; Humblet, C. J. Med. Chem. 2009, 52, 6752. (b) Lovering, F. MedChemComm 2013, 4, 515.

(9) Eaton, P. E. Angew. Chem., Int. Ed. Engl. 1992, 31, 1421.

(10) Aldeghi, M.; Malhotra, S.; Selwood, D. L.; Chan, A. W. E. Chem. Biol. Drug Des. 2014, 83, 450.

(11) (a) Mykhailiuk, P. K. Org. Biomol. Chem. 2019, 17, 2839. (b) Ma, X.; Pham, L. N. Asian J. Org. Chem. 2020, 8-22.

(12) Pekker, S.; Kovats, E.; Oszlanyi, G.; Benyei, G.; Klupp, G.; Bortel, G.; Ialsovszky, I.; Iakab, E.; Borondics, F.; Kamaras, K.; Bokor, M.; Kriza, G.; Tompa, K.; Faigel, G. Nat. Mater. 2005, 4, 764.

(13) Sakai, N.; Mareda, J.; Matile, S. Acc. Chem. Res. 2005, 38, 79

(14) Zimmerman, H. E.; McKelvey, R. D. J. Am. Chem. Soc. 1971, 93, 3638.

(15) (a) Locke, G. M.; Senge M. 0. ECS Trans. 2016, 72, 1. (b) R. M. Metzger, J. Mater. Chem. 2008, 18, 4364.

(16) (a) Jana, R.; Pathak, T. P.; Sigman, M. S. Chem. Rev. 2011, 111, 1417. (b) Tasker, S. Z.; Standley, E. A.; Jamison, T. F. Nature 2014, 509, 299.

(17) He, J.; Wasa, M.; Chan, K. S. L.; Shao, Q.; Yu, J.-Q. Chem. Rev. 2017, $117,8754$.

(18) (a) Smith, J. M.; Harwood, S. J.; Baran, Phil S. Acc. Chem. Res. 2018, 51, 1807. (b) Murarka, S. Adv. Synth. Catal 2018, 360, 1735.

(19) (a) Hoffmann, R.; Hopf, H. Angew. Chem. Int. Ed. 2008, 47, 4474. (b) Schleyer, P. v. R.; Williams, J. E. J. Am. Chem. Soc. 1970, 92, 2377.

(20) Weltner, W. J. Am. Chem. Soc. 1953, 75, 4224.

(21) (a) Freedman, H. H. J. Am. Chem. Soc. 1961, 83, 2194. (b) Freedman, H. H.; Petersen, D. R. J. Am. Chem. Soc. 1962, 84, 2837.

(22) Throndsen, H. P.; Zeiss, H. J. Organomet. Chem. 1964, 1, 301

(23) (a) Eaton, P. E.; Cole, T. W. J. Am. Chem. Soc. 1964, 86, 962.

(24) Chapman, N. B.; Key, J. M.; Toyne, K. J. J. Org. Chem. 1970, 35, 3860.

(25) Falkiner, M. J.; Littler, S. W.; McRae, K. J.; Savage, G. P.; Tsanaktsidis, J. Org. Process Res. Dev. 2013, 17, 1503.

(26) Eaton, P. E.; Cole, T. W. J. Am. Chem. Soc. 1964, 86, 3157.

(27) Gleiter, R.; Karcher, M. Angew. Chem. 1988, 100, 851.

(28) Pelosi, L. F.; Miller, W. T. J. Am. Chem. Soc. 1976, 98, 4311.

(29) de Meijere, A.; Redlich, S.; Frank, D.; Magull, J.; Hofmeister, A.; Menzel, H.; Konig, B.; Svoboda, J. Angew. Chem., Int. Ed. 2007, 46, 4574.

(30) Chalmers, B. A.; Xing, H.; Houston, S.; Clark, C.; Ghassabian, S.; Kuo, A.; Cao, B.; Reitsma, A.; Murray, C.-E. P.; Stok, J. E.; Boyle, G. M.; Pierce, C. J.; Littler, S. W.; Winkler, D. A.; Bernhardt, P. V.; Pasay, C.; DeVoss, J. J.; McCarthy, J.; Parsons, P. G.; Walter, G. H.; Smith, M. T.; Cooper, H. M.; Nilsson, S. K.; Tsanaktsidis, J.; Savage, G. P.; Williams, C. M. Angew. Chem. Int. Ed. 2016, 55, 3580.

(31) Tristan, A. R.; Williams, C. M.; Rendina, L. M.; Kassiou, M. J. Med. Chem., 2019, 62, 1078.

(32) (a) Luh, T. Y.; Stock, L. M. J. Am. Chem. Soc. 1974, 96, 3712. (b) Srinivasan, S. K.; Bläser, D.; Boese, R.; Desiraju, G. R. J. Org. Chem. 2001, 66, 1621. (c) Flanagan, K. J.; Bernhard, S. S. R.; Plunkett, S.; Senge, M. O. Chem. Eur. J. 2019, 25, 6941. (d) Macreadie, L. K.; Mensforth, E. J.; Babarao, R.; Konstas, K.; Telfer, S. G.; Doherty, C. M.; Tsanaktsidis, J.; Batten, S. R.; Hill, M. R. J. Am. Chem. Soc. 2019, $141,3828$.

(33) (a) Eaton, P. E.; Zhang, M. X.; Gilardi, R.; Gelber, N.; Iyer, S.; Surapaneni, R. Prop., Explos., Pyrotech. 2002, 27, 1. (b) Zhang, M.X.; Eaton, P. E.; Gilardi, R. Angew. Chem. Int. Ed. 2000, 39, 401. (c) Dallaston, M. A.; Brusnahan, J. S.; Wall, C.; Williams. C. M. Chem. Eur. J. 2019, 25, 8344.

(34) Kybett, B. D.; Carroll, S.; Natalis, P.; Bonnell, D. W.; Margrave, J. L.; Franklin, J. L. J. Am. Chem. Soc. 1966, 88, 626.
(35) Sollott, G. P.; Alster, J.; Gilbert, E. E.; Sandus, O.; Slagg, N. J. Energ. Mater. 1986, 4, 5

(36) Eaton, P. E.; Ravi-Shankar, B. K.; Price, G. D.; Pluth, J. J.; Gilbert, E. E.; Alster, J.; Sandus, O. J. Org. Chem. 1984, 49, 185.

(37) Eaton, P. E.; Wicks, G. E. J. Org. Chem. 1988, 53, 5353

(38) Lukin, K. A.; Li, J.; Eaton, P. E.; Kanomata, N.; Hain, J.; Punzalan, E.; Gilardi, R. J. Am. Chem. Soc. 1997, 119, 9591.

(39) (a) Bashir-Hashemi, A. Angew. Chem., Int. Ed. Engl. 1993, 32, 612. (b) Bashir-Hashemi, A.; Li, J.; Gelber, N.; Ammon, H. J. Org. Chem. 1995, 60, 698

(40) Moriarty, R. M.; Khosrowshahi, J. S.; Dalecki, T. M. J. Chem. Soc., Chem. Commun., 1987, 675.

(41) (a) Barton, D. H. R.; Lacher, B.; Zard, S. Z. Tetrahedron, 1987, 43, 4321. (b) Barton, D. H. R.; Crich, D.; Motherwell, W. B. Tetrahedron Lett. 1983, 24, 4979 .

(42) Tsanaktsidis, J.; Eaton, P. E. Tetrahedron Lett. 1989, 30, 6967.

(43) (a) Reddy, D. S.; Sollott, G. P.; Eaton, P. E. J. Org. Chem. 1989, 54, 722. (b) Irngartinger, H.; Strack, S.; Gredel, F.; Dreuw, A.; Della, E. W. Eur. J. Org. Chem. 1999, 1253.

(44) Eaton, P. E.; Maggini, M. J. Am. Chem. Soc. 1988, 110, 7230.

(45) (a) Capriati, V.; Perna, F. M.; Salomone, A. Dalton Trans. 2014, 43, 14204.(b) Eaton, P. E.; Tsanaktsidis, J. J. Am. Chem. Soc. 1990, 112, 876.

(46) Eaton, P. E.; Pramod, K.; Emrick, T.; Gilardi R. J. Am. Chem. Soc. 1999, 121, 4111

(47) Eaton, P. E.; Zhou, J. P. J. Am. Chem. Soc. 1992, 114, 3118. (b) Eaton, P. E.; Yang, C. X.; Xiong, Y. J. Am. Chem. Soc., 1990, 112, 3225.

(48) Kato, Y.; Williams, C. M.; Uchiyama, M.; Matsubara, S. Org. Lett. 2019, 21, 473.

(49) Plunkett, S.; Flanagan, K. J.; Twamley, B.; Senge, M. 0 . Organometallics 2015, 34, 1408.

(50) (a) Schwab, P. F. H.; Levin, M. D.; Michl, J. Chem. Rev. 1999, 99, 1863. (b) Schwab, P. F. H.; Smith, J. R.; Michl, J. Chem. Rev. 2005 , $105,1197$.

(51) Negishi, E.; King, A. O.; Klima, W. L.; Patterson, W.; Silveira, A. J. Org. Chem. 1980, 45, 2526.

(52) Eaton, P. E.; Stossel, D. J. Org. Chem. 1991, 56, 5138.

(53) (a) Eaton, P. E.; Galoppini, E. Gilardi, R. J. Am. Chem. Soc. 1994, 116, 7588. (b) Houston, S. D.; Xing, H.; Bernhardt, P. V.; Vanden Berg, T. J.; Tsanaktsidis, J.; Savage, G. P.; Williams, C. M. Chem. Eur. J. 2019, 25, 2735

(54) Bernhard, S. S. R.; Locke, M. G.; Plunkett, S.; Meindl, A.; Flanagan, K. J.; Senge, M. O. Chem. Eur. J. 2018, 24, 1026.

(55) Hoffmann, R.; Woodward, R. B. Acc. Chem. Res. 1968, 1, 17.

(56) (a) Eaton, P. E.; Cassar, L.; Halpern, J. J. Am. Chem. Soc. 1970, 92, 6366. (b) Moss, S.; King, B. T.; de Meijere, A.; Kozhushkov, S. I.; Eaton, P. E.; Michl, J. Org. Lett. 2001, 3, 2375. (c) Jalife, S.; Mondal, S.; Cabellos, J. L.; Martinez-Guajardo, G.; Fernandez-Herrera, M. A.; Merino, G. Chem. Commun. 2016, 52, 3403.

(57) (a) Hassenrück, K.; Radziszewski, J. G.; Balaji, V.; Murthy, G. S.; McKinley, A. J.; David, D. E.; Lynch, V. M.; Martin, H.-D.; Michl, J. J. Am. Chem. Soc. 1990, 112, 873. (b) Borden, W. T.; Hrovat, D. A. J. Am. Chem. Soc. 1990, 112, 875.

(58) Moriarty, R. M.; Khosrowshahi, J. S.; Miller, R. S.; FlippenAndersen, J.; Gilardi, R. J. Am. Chem. Soc. 1989, 111, 8943.

(59) Okada, K.; Okamoto, K.; Morita, N.; Okubo, K.; Oda, M. J. Am. Chem. Soc. 1991, 113, 9401

(60) Toriyama, F.; Cornella, J.; Wimmer, L.; Chen, T.-G.; Dixon, D.; Creech, G.; Baran, P. S. J. Am. Chem. Soc. 2016, 138, 11132.

(61) Fawcett, A.; Pradeilles, J.; Wang, Y.; Mutsuga, T.; Myers, E. L.; Aggarwal, V. K. Science 2017, 357, 283.

(62) Collin, D. E.; Folgueiras-Amador, A. A.; Pletcher, D.; Light, M. E.; Linclau, B.; Brown, R. C. D. Chem. Eur. J. 2020, 20, 374

(63) (a) Pellicciari, R.; Costantino, G.; Giovagnoni, E.; Mattoli, L.; Brabet, I.; Pin, J.-P. Bioorg. Med. Chem. Lett. 1998, 8, 1569. (b) Curry, K. PCT Int. Appl, WO 2004024709A2, 2004. (c) Houston, S. D.; Chalmers, B. A.; Savage, G. P.; Williams, C. M. Org. Biomol. Chem. 2019, 17, 1067. (d) Wlochal, J.; Davies, R. D. M.; Burton, J. Org. 
Lett. 2014, 16, 4094. (e) Grover, N.; Locke, G. M.; Flanagan, K. J.; Beh, M. H. R.; Thompson, A.; Senge, M. O. Chem. Eur. J. 2020, 26 2405.

(64) Luh, T.-Y.; Stock, L. M. J. Am. Chem. Soc. 1974, 96, 3712.

(65) Pati, R.; Karna, S. P. J. Chem. Phys. 2001, 115, 1703.

(66) Lancaster, J. R.; Martí, A. A.; López-Gejo, J.; Jockusch, S.; O'Connor, N.; Turro, N. J. Org. Lett. 2008, 10, 5509.

(67) Wiberg, K. B. Tetrahedron Lett. 1985, 26, 599.

(68) Wiberg, K. B.; Connor, D. S.; Lampmann, G. M. Tetrahedron Lett. 1964, $5,531$.

(69) Wiberg, K. B.; Connor, D. S. J. Am. Chem. Soc. 1966, 88, 4437.

(70) Meinwald, J.; Szkrybalo, W.; Dimmel, D. R. Tetrahedron Lett. 1967 $8,731$.

(71) Applequist, D. E.; Wheeler, J. W. Tetrahedron Lett. 1977, 18, 3411.

(72) Wiberg, K. B.; Walker, F. H. J. Am. Chem. Soc. 1982, 104, 5239.

(73) (a) Semmler, K.; Szeimies, G.; Belzner, J. J. Am. Chem. Soc. 1985, 107, 6410. (b) Lynch, K. M.; Dailey, W. P. J. Org. Chem. 1995, 60, 4666. (c) Mondanaro, K. R.; Dailey, W. P. Org. Synth. 1998, 75, 98.

(74) (a) Stohrer, W. D.; Hoffmann, R.; J. Am. Chem. Soc. 1972, 94, 779. (b) Wiberg, K. B. J. Am. Chem. Soc. 1983, 105, 1227. (c) Chakrabarti, P.; Seiler, P.; Dunitz, J. D.; Schlüter, A. D.; Szeimies, G. J. Am. Chem. Soc. 1981, 103, 7378. (d) Jackson, J. E.; Allen, L. C. J. Am. Chem. Soc. 1984, 106, 59. (e) Werner, M.; Stephenson, D. S.; Szeimies, G. Liebigs Ann. Chem. 1996, 1705. (f) Wu, W.; Gu, J.; Song, J.; Shaik, S.; Hiberty, P. C. Angew. Chem. Int. Ed. 2009, 48, 1407.

(75) (a) Wiberg, K. B.; Waddell, S. T. J. Am. Chem. Soc. 1990, 112, 2194. (b) Delia, E. W.; Lochert, I. J. Org. Prep. Proceed. Int. 1996, 28, 411.

(76) (a) Kaszynski, P.; McMurdie, N. D.; Michl, J. J. Org. Chem. 1991, 56, 307. (b) Wiberg, K. B.; McMurdie, N. J. Am. Chem. Soc. 1994, 116, 11990.

(77) Hossain, Md. T.; Timberlake, J. W. J. Org. Chem. 2001, 66, 4409.

(78) Wiberg, K. B.; Waddell, S. T. Tetrahedron Lett. 1988, 29, 289.

(79) Mazal, C.; Paraskos, A. J.; Michl, J. J. Org. Chem. 1998, 63, 2116.

(80) Messner, M.; Kozhushkov, S. I.; de Meijere, A. Eur. J. Org. Chem. 2000, 1137.

(81) (a) Caputo, D. F. J.; Arroniz, C.; Dürr, A. B.; Mousseau, J. J.; Stepan, A. F.; Mansfield, S. J.; Anderson, E. A. Chem. Sci., 2018, 9, 5295. (b) Wong, M. L. J.; Mousseau, J. J.; Mansfield, S. J.; Anderson, E. A. Org. Lett. 2019, 21, 4395. (c) Nugent, J.; Arroniz, C.; Shire, B. R.; Sterling, A. J.; Pickford, H. D.; Wong, M. L. J.; Mansfield, S. J.; Caputo, D. F. J.; Owen, B.; Mousseau, J. J.; Duarte, F.; Anderson, E. A. ACS Catal. 2019, 9, 9568.

(82) (a) Schwab, P. F. H.; Noll, B. C.; Michl, J. J. Org. Chem. 2002, 67, 5476. (b) Kaleta, J.; Michl, J.; Mézière, C.; Simonov, S.; Zorina, L.; Wzietek, P.; Rodríguez-Fortea, A.; Canadell, E.; Batail, P. CrystEngComm 2015, 17, 7829. (c) Kaleta, J.; Janoušek, Z.; Nečas, M.; Mazal, C. Organometallics 2015, 34, 967.

(83) (a) Bunz, U.; Szeimies, G. Tetrahedron Lett. 1989, 30, 2087. (b) Kaleta, J.; Mazal, C. Org. Lett. 2011, 13, 1326. (c) Kaleta, J.; Marek, N.; Mazal, C. Eur. J. Org. Chem. 2012, 4783. (d) Kaleta, J.; Bastien, G.; Císařová, I.; Batail, P.; Michl, J. Eur. J. Org. Chem. 2018, 2018, 5137.

(84) Kaleta, J.; Bastien, G.; Wen, J.; Dračínský, M.; Tortorici, E.; Císařová, I.; Beale, P. D.; Rogers, C. T.; Michl, J. J. Org. Chem. 2019, 84, 8449.

(85) (a) Rodríguez-Fortea, A.; Kaleta, J.; Mézière, C.; Allain, M.; Canadell, E.; Wzietek, P.; Michl, J.; Batail, P. ACS Omega, 2018, 3, 1293. (b) Lemouchi, C.; Vogelsberg, C. S.; Zorina, L.; Simonov, S.; Batail, P.; Brown, S.; Garcia-Garibay, M. A. J. Am. Chem. Soc. 2011, $133,6371$.

(86) (a) Rehm, J. D. D.; Ziemer, B.; Szeimies, G. Eur. J. Org. Chem. 1999, 2079. (b) Rehm, J. D. D.; Ziemer, B.; Szeimies, G. Eur. J. Org. Chem. 2001, 1049. (c) Makarov, S.; Brocklehurst, C. E.; Karaghiosoff, K.; Koch, G.; Knochel, P. Angew. Chem. Int. Ed., 2017, 56, 12774. (c) Kondo, M.; Kanazawa, J.; Ichikawa, T.; Shimokawa, T.; Nagashima, Y.; Miyamoto, K.; Uchiyama, M. Angew. Chem. Int. Ed. 2020, 59, 1970. (d) Yu, S.; Jing, C.; Noble, A.; Aggarwal, V. K. Angew. Chem. Int. Ed. 2020, 59, 3917. (e) Garlets, Z. J.; Sanders, J. N.; Malik, H.;
Gampe, C.; Houk, K. N.; Davies, H. M. L. Nat. Catal., 2020, DOI: 10.1038/s41929-019-0417-1

(87) (a) Lasányi, D.; Tolnai, G. L. Org. Lett. 2019, 21, 10057. (b) Yu, S.; Noble, A.; Bedford, R. B.; Aggarwal, V. K. J. Am. Chem. Soc. 2019, 141, 20325.

(88) (a) Wiberg, K. B.; Williams, V. Z. J. Org. Chem. 1970, 35, 369. (b) Della, E. W.; Kasum, B.; Kirkbride, K. P. J. Am. Chem. Soc. 1987, 109, 2746. (c) Toops, D. S.; Barbachyn, M. R. J. Org. Chem. 1993 58, 6505. (d) Bunker, K. D.; Sach, N. W.; Huang, Q.; Richardson, P. F. Org. Lett. 2011, 13, 4746. (e) Thirumoorthi, N. T.; Shen, C. J.; Adsool, V. A. Chem. Commun., 2015, 51, 3139. (f) Goh, Y. L.; Tam, E. K. W.; Bernardo, P. H.; Cheong, C. B.; Johannes, C. W.; William, A. D.; Adsool, V. A. Org. Lett. 2014, 16, 1884.

(89) (a) Gianatassio, R.; Lopchuk, J. M.; Wang, J.; Pan, C.-M.; Malins, L. R.; Prieto, L.; Brandt, T. A.; Collins, M. R.; Gallego, G. M.; Sach, N. W.; Spangler, J. E.; Zhu, H.; Zhu, J.; Baran, P. S. Science 2016, 351, 241. (b) Lopchuk, J. M.; Fjelbye, K.; Kawamata, Y.; Malins, L. R.; Pan, C.-M.; Gianatassio, R.; Wang, J.; Prieto, L.; Bradow, J.; Brandt, T. A.; Collins, M. R.; Elleraas, J.; Ewanicki, J.; Farrell, W.; Fadeyi, O. O.; Gallego, G. M.; Mousseau, J. J.; Oliver, R.; Sach, N. W.; Smith, J. K.; Spangler, J. E.; Zhu, H.; Zhu, J.; Baran, P. S. J. Am. Chem. Soc. 2017, 139, 3209. (c) Kanazawa, J.; Maeda, K.; Uchiyama, M. J. Am. Chem. Soc. 2017, 139, 17791. (d) Shelp, R. A.; Walsh, P. J. Angew. Chem. Int. Ed. 2018, 57, 15857. (e) Hughes, J. M. E.; Scarlata, D. A.; Chen, A. C.-Y.; Burch, J. D.; Gleason, J. L. Org. Lett. 2019, 21, 6800.

(90) (a) Ni S.; Garrido-Castro ${ }_{\llcorner}$A. F; Merchant, R. R.; de Gruyter, J. N.; Schmitt, D. C.; Mousseau, J. J.; Gallego, G. M.; Yang, S.; Collins, M. R.; Qiao, J. X.; Yeung, K. S.; Langley, D. R.; Poss, M. A.; Scola, P. M.; Qin, T.; Baran, P. S. Angew. Chem. Int. Ed. 2018, 57, 14560. (b) Liang, Y.; Zhang, X.; MacMillan, D.W.C. Nature 2018, 559, 83. (c) VanHeyst, M. D.; Qi, J.; Roecker, A. J.; Hughes, J. M. E.; Cheng, L.; Zhao, Z.; Yin, J. Org. Lett. 2020, 22, 1648. (d) Xiaheng, Z.; Russell T. S.; Chip L.; Stefan J. M.; Brock, T. S.; Nicholas, I. C.; David, W. C. M. Nature 2020, DOI: 10.1038/s41586-020-2060-z. (e) Kim, J. H.; Ruffoni, A.; Al-Faiyz, Y. S. S.; Sheikh, N. S.; Leonori, D. Angew. Chem. Int. Ed. 2020, DOI: 10.1002 /anie.202000140.

(91) (a) Bär, R. M.; Kirschner, S.; Nieger, M.; Bräse, S. Chem. Eur. J. 2018, 24, 1373. (b) Bär, R. M.; Heinrich, G.; Nieger, M.; Fuhr, O.; Bräse, S. Beilstein J. Org. Chem. 2019, 15, 1172. (c) Bär, R. M.; Langer, L.; Nieger, M.; Bräse S. Adv. Synth. Catal. 2020, 362, 1356.

(92) (a) Trongsiriwat, N.; Pu, Y.; Nieves-Quinones, Y.; Shelp, R. A.; Kozlowski, M. C.; Walsh, P. J. Angew. Chem. Int. Ed. 2019, 58, 13416. (b) Rout, S. K.; Marghem, G.; Lan, J.; Leyssens, T.; Riant, O. Chem. Commun., 2019, 55, 14976.

(93) de Meijere, A.; Zhao, L.; Belov, V. N.; Bossi, M.; Noltemeyer, M.; Hell, S. W. Chem. Eur. J., 2007, 13, 2503.

(94) (a) Levin, M. D.; Hamrock, S. J.; Kaszynski, P.; Shtarev, A. B.; Levina, G. A.; Noll, B. C.; Ashley, M. E.; Newmark, R.; Moore, G. G. I.; Michl, J. J. Am. Chem. Soc. 1997, 119, 12750. (b) Shtarev, A. B.; Pinkhassik, E.; Levin, M. D.; Stibor, I.; Michl, J. J. Am. Chem. Soc. 2001, 123, 3484. (c) Kaleta, J.; Rončević, I.; Císařová, I.; Dračínský, M.; Šolínová, V.; Kašička, V.; Michl, J. J. Org. Chem. 2019, 84, 2448.

(95) (a) Ma, X.; Sloman, D. L.; Han, Y.; Bennett, D. J. Org Lett. 2019, 21, 7199.(b) Bychek, R. M.; Hutskalova, V.; Bas, Y. P.; Zaporozhets, O. A.; Zozulya, S.; Levterov, V. V.; Mykhailiuk, P. K. J. Org. Chem. 2019, $84,15106$.

(96) Wanka, L.; Iqbal, K.; Schreiner, P. R. Chem. Rev. 2013, 113, 3516.

(97) (a) Landa, S.; Machacek, V. Collect. Czech. Chem. C. 1933, 5, 1. (b) Rollmann, L. D.; Green, L. A.; Bradway, R. A.; Timken, H. K. C. Catal. Today, 1996, 31, 163.

(98) (a) Schleyer, P. v. R. J. Am. Chem. Soc. 1957, 79, 3292. (b) Fort, R. C.; Schleyer, P. v. R. Chem. Rev. 1964, 64, 277.

(99) (a) Nekrasova, V.A.; Shuikin, I. I. Russ Chem Bull 1969, 18, 649. (b) Olah, G. A.; Lin, H. C. H. J. Am. Chem. Soc. 1971, 93, 1259. (c) Akhrem, I. S.; Avetisyan, D. V.; Afanas'eva, L. V.; Goryunov, E. I.; Churilova, I. M.; Petrovskii, P. V.; Kagramanov, N. D. Mendeleev Commun. 2011, 21, 259. (d) Reichert, V. R.; Mathias, L. J. Macromolecules 1994, 27, 7015. 
(100) (a) Webber, W. C.; Harthoorn, P. A. Br. Pat. Appl. 8192401959. (b) Zapf, A.; Ehrentraut, A.; Beller, M. Angew. Chem. Int. Ed. 2000 39, 4153. (c) Agnew-Francisa, K. A.; Williams, C. M. Adv. Synth. Catal. 2016, 358, 675.

(101) Egloff, G.; Schaad, R. E.; Lowry, C. D. Chem. Rev. 1931, 8, 1.

(102) (a) Smith, G. W.; Williams, H. D. J. Org. Chem. 1961, 26, 2207. (b) Baughman, G. L. J. Org. Chem.1964, 29, 238. (c) Murray, R. W.; Rajadhyaksha, S. N.; Mohan, L. J. Org. Chem. 1989, 54, 5783.

(103) (a) Stetter, H.; Schwarz, M.; Hirschhorn, A. Angew. Chem. 1959, 71, 429. (b) Sasaki, T.; Eguchi, S.; Toru, T. Bull. Korean Chem. Soc. 1968, 41, 236.

(104) Ohno, M.; Ishizaki, K.; Eguchi, S. J. Org. Chem. 1988, 53, 1285.

(105) Bräse, S.; Waegell, B.; de Meijere, A. Synthesis 1998, 1998, 148.

(106) Someya, H.; Yorimitsu, H.; Oshima, K. Tetrahedron Lett. 2009, 50, 3270.

(107) Ghorai, S. K.; Jin, M.; Hatakeyama, T.; Nakamura, M. Org. Lett. 2012, 14, 1066.

(108) (a) Zultanski, S. L.; Fu, G. C. J. Am. Chem. Soc. 2013, 135, 624. (b) Pouwer, R. H.; Williams, C. M.; Raine, A. L.; Harper, J. B. Org. Lett. 2005, 7, 1323. (c) Pouwer, R. H.; Harper, J. B.; Vyakaranam, K.; Michl, J.; Williams, C. M.; Jessen, C. H.; Bernhardt, P. V. Eur. J. Org. Chem. 2007, 241

(109) Molle, G.; Bauer, P.; Dubois, J. E. J. Org. Chem. 1982, 47, 4120.

(110) Rieke, R. D.; Hanson, M. V.; Brown, J. D. J. Org. Chem. 1996, 61, 2726.

(111) Lohre, C.; Dröge, T.; Wang, C.; Glorius, F. Chem. Eur. J. 2011, 17, 6052.

(112) (a) Sämann, C.; Dhayalan, V.; Schreiner, P. R.; Knochel, P. Org. Lett. 2014, 16, 2418. (b) Milne, J. E.; Buchwald, S. L. J. Am. Chem. Soc. 2004, 126, 13028.

(113) Perry, I. B.; Brewer, T. F.; Sarver, P. J.; Schultz, D. M.; DiRocco, D. A.; MacMillan, D. W. C. Nature 2018, 560, 70.

(114) Berndt, J.-P.; Erb, F.; Ochmann, L.; Beppler, J.; Schreiner, P. Synlett 2019, 30, 493.

(115) Yang, H.-B.; Feceu, A.; Martin, D. B. C. ACS Catal. 2019, 9, 5708.

(116) (a) Tominaga, M.; Kunitomi, N.; Katagiri, K.; Itoh, T. Org. Lett. 2015, 17, 786. (b) Tominaga, M.; Takahashi, E.; Ukai, H.; Ohara, K.; Itoh, T.; Yamaguchi, K. Org. Lett. 2017, 19, 1508. (c) Tominaga, M.; Takahashi, E.; Ukai, H.; Ohara, K.; Itoh, T.; Yamaguchi, K. J. Org. Chem. 2019 84, 5109.

(117) Kahl, P.; Wagner, J. P.; Balestrieri, C.; Becker, J.; Hausmann, H.; Bodwell, G. J.; Schreiner, P. v. R. Angew. Chem. Int. Ed. 2016, 128, 9423.

(118) Basel, B. S.; Zirzlmeier, J.; Hetzer, C.; Phelan, B. T.; Krzyaniak, M. D.; Reddy, S. R.; Coto, P. B.; Horwitz, N. E.; Young, R. M.; White, F. J.; Hampel, F.; Clark, T.; Thoss, M.; Tykwinski, R. R.; Wasielewski, M. R.; Guldi, D. M. Nat. Commun., 2017, 8, 15171.

(119) (a) Pathak, R.; Marx, A. Chem. Asian. J. 2011, 6, 1450. (b) Štimac, A.; Šekutor, M.; Mlinarić-Majerski, K.; Frkanec, L.; Frkanec, R. Molecules 2017, 22, 297.

(120) (a) Newman, H. Synthesis 1972, 692. (b) Pannier, N.; Maison, W. Eur. J. Org. Chem. 2008, 1278.
(121) (a) Radhakrishnan, U.; Schweiger, M.; Stang, P. J. Org. Lett. 2001 3, 3141. (b) Trawny, D.; Kunz, V.; Reissig, H.-U. Eur. J. Org. Chem. 2014, 6295 .

(122) Tominaga, M.; Ohara, K.; Yamaguchi, K.; Azumaya, I. J. Org. Chem. 2014, 79, 6738.

(123) (a) Grigg, R.; Elboray, E. E.; Alyb, M. F.; Abbas-Temirekb, H. H. Chem. Commun. 2012, 48, 11504. (b) Kitagawa, T.; Matsubara, H.; Komatsu, K.; Hirai, K.; Okazaki, T.; Hase, T. Langmuir, 2013, 29, 4275. (c) Franzmann, E.; Khalil, F.; Weidmann, C.; Schrçder, M.; Rohnke, M.; Janek, J.; Smarsly, B. M.; Maison, W. A. Chem. Eur. J. 2011, 17, 8596. (d) Senchyk, G. A.; Lysenko, A. B.; Boldog, I.; Rusanov, E. B.; Chernega, A. N.; Krautscheidc, H.; Domasevitch, K. V. Dalton Trans., 2012, 41, 8675.

(124) Lee, G. S.; Bashara, J. N.; Sabih, G.; Oganesyan, A.; Godjoian, G.; Duong, H. M.; Marinez, E. R.; Gutiérrez, C. G. Org. Lett. 2004, 6, 1705.

(125) (a) Sollott, G. P.; Gilbert, E. E. J. Org. Chem.1980, 45, 5405. (b) Otto E. J. Am. Chem. Soc. 1988, 110, 3141. (c) Bashir-Hashemi, A.; Jianchang L. Tetrahedron Lett. 1995, 36, 1233.

(126) (a) Mathias, L. J.; Reichert, V. R.; Muir, A. V. G. Chem. Mater., 1993, 5, 4-5. (b) Reichert, V. R.; Mathias, L. J. Macromolecules 1994, 27, 7015.

(127) (a) Schilling, C. I.; Plietzsch, O.; Nieger, M.; Muller, T.; Bräse, S. Eur. J. Org. Chem., 2011, 1743. (b) Schilling, C. I.; Bräse, S. Org. Biomol. Chem., 2007, 5, 3586. (c) Plietzsch, O.; Schilling, C. I.; Tolev, M.; Nieger, M.; Richert, C.; Muller, T.; Bräse, S. Org. Biomol. Chem., 2009, 7, 4734. (d) Grigg, R.; Elboray, E. E.; Aly, M. F.; AbbasTemirek, H. H. Chem. Commun., 2012, 48, 11504.

(128) Dufrêne, Y. F.; Ando, T.; Garcia, R.; Alsteens, D.; Martinez-Martin, D.; Engel, A.; Gerber, C.; Müller, D. J. Nat. Nanotechnol. 2017, 12, 295.

(129) Li, Q.; Rukavishnikov, A. V.; Petukhov, P. A.; Zaikova, T. O.; Jin, C.; Keana, J. F. W. J. Org. Chem. 2003, 68, 4862.

(130) Martin, V. V.; Alferiev, I. S.; Weis, A. L. Tetrahedron Lett. 1999, 40, 223.

(131) Hetzer, C.; Basel, B. S.; Kopp, S. M.; Hampel, F.; White, F. J.; Clark, T.; Guldi, D. M.; Tykwinski, R. R. Angew. Chem. Int. Ed. 2019, 58, 15263.

(132) (a) Humblet, V.; Misra, P.; Bhushan, K. R.; Nasr, K.; Ko, Y. S.; Tsukamoto, T.; Pannier, N.; Frangioni, J. V.; Maison, W. J. Med. Chem. 2009, 52, 544. (b) Pavet, V.; Beyrath, J.; Pardin, C.; Morizot, A.; Lechner, M. C.; Briand, J. P.; Wendland, M.; Maison, W.; Fournel, S.; Micheau, O.; Guichard, G.; Gronemeyer, H. Cancer Res. 2010, 70, 1101. (c) Franzmann, E.; Khalil, F.; Weidmann, C.; Schröder, M.; Rohnke, M.; Janek, J.; Smarsly, B. M.; Maison, W. Chem. Eur. J. 2011, 17, 8596. (d) Maison, W.; Frangioni, J. V.; Pannier, N. Org. Lett. 2004, 6, 4567 .

(133) (a) Carsten F.; Elisa F.; Dorith C.; Aljona R.; Wolfgang M. Synthesis 2013, 45, 1452. (b) Lamanna, G.; Russier, J.; Ménard-Moyon, C.; Bianco, A. Chem. Commun., 2011, 47, 8955. 


\section{Biosketches}

Nitika Grover received her Ph.D from Indian Institute of Technology Roorkee (IITR) in 2017. Her
doctoral research with Prof. M. Sankar was focused on the synthesis of non-planar porphyrins and
chlorins. In 2018, she joined the research group of Prof. Dr Mathias O. Senge as a post doctorate fellow.
Currently, she is working on the CH-activation of highly strained hydrocarbons.

